\title{
An Epidemiological Assessment of Lung Function Decline as a Predictor of Morbidity and Mortality
}

\author{
Penelope J. Baughman \\ West Virginia University
}

Follow this and additional works at: https://researchrepository.wvu.edu/etd

\section{Recommended Citation}

Baughman, Penelope J., "An Epidemiological Assessment of Lung Function Decline as a Predictor of Morbidity and Mortality" (2011). Graduate Theses, Dissertations, and Problem Reports. 4689.

https://researchrepository.wvu.edu/etd/4689

This Dissertation is protected by copyright and/or related rights. It has been brought to you by the The Research Repository @ WVU with permission from the rights-holder(s). You are free to use this Dissertation in any way that is permitted by the copyright and related rights legislation that applies to your use. For other uses you must obtain permission from the rights-holder(s) directly, unless additional rights are indicated by a Creative Commons license in the record and/ or on the work itself. This Dissertation has been accepted for inclusion in WVU Graduate Theses, Dissertations, and Problem Reports collection by an authorized administrator of The Research Repository @ WVU.

For more information, please contact researchrepository@mail.wvu.edu. 


\title{
An Epidemiological Assessment of Lung Function Decline as a Predictor of Morbidity and Mortality
}

\author{
Penelope J. Baughman \\ Dissertation submitted to the \\ School of Medicine \\ at West Virginia University \\ in partial fulfillment of the requirements for the degree of \\ Doctor of Philosophy
in
Public Health Sciences \\ Eva Hnizdo, PhD \\ Michael E. Andrew, PhD \\ Christopher J. Martin, MD, MSc \\ Edward L. Petsonk, MD \\ Anoop Shankar, MD, PhD \\ Department of Community Medicine
}

Morgantown, West Virginia

2011

Keywords: Spirometry, Respiratory Symptoms, Chronic Obstructive Pulmonary Disease, Morbidity, Mortality 


\section{ABSTRACT \\ An Epidemiological Assessment of Lung Function Decline as a Predictor of Morbidity and Mortality}

\section{Penelope J. Baughman}

Excessive lung function decline is associated with increased risks of respiratory morbidity and mortality outcomes including chronic obstructive pulmonary disease (COPD). COPD is a disease afflicting approximately 80 million people worldwide and as of 2002, the fifth leading cause of death. COPD is an increasing problem, with substantial increases projected in COPD deaths if there is no reduction of the causal risk factors. By 2030, COPD is projected to be the third leading cause of death worldwide, an increase mainly due to extended life expectancies and that COPD deaths occur at older ages. COPD is also largely underdiagnosed, partially due to the gradual development of the disease, and is a very costly illness that reduces labor force participation in its more severe stages. Existing studies of lung function decline as a predictor of morbidity and mortality are focused upon assessing lung function decline as an independent risk factor among other known risk factors. The objective of this study was to assess lung function as a predictor of morbidity and mortality in minimally-adjusted models to provide information for prevention purposes of both lung function decline and associated morbidity and mortality outcomes. The population-based Copenhagen City Heart Study data (1976-2003) were used to investigate this objective. These data were fitting because many of the study participants were at risk for lung function decline due to high rates of smoking and the study also offered long follow-up for COPD morbidity, and mortality due to COPD, coronary heart disease, and allcauses. The first study was designed to examine the relative contributions of lung function decline and respiratory symptoms in predicting the adverse health outcomes. The first study relates to workplace respiratory disease prevention programs where spirometry testing and a respiratory symptom questionnaire are often used. The results of the first study indicated that lung function decline was associated with increased risks of COPD morbidity and mortality, that the effect was greater than for respiratory symptoms and asthma, emphasizing the potential usefulness of monitoring for lung function change over time for prevention in at-risk occupational populations. The second study investigated morbidity and mortality risks in two areas that are not well investigated: in younger individuals, and in relation to long-term patterns

of lung function decline. The results indicated that increased lung function decline in individuals age 45 years or younger and the persistency of accelerated lung function decline over follow-up time were significant predictors for increased respiratory morbidity and mortality. The third study evaluated whether the combined effect of the level of lung function at baseline and subsequent rate of lung function decline could further identify high-risk groups. The results showed that the combined effect of a decreasing level of baseline forced expiratory volume in one second $\left(\mathrm{FEV}_{1 \mathrm{~b}}\right)$ and increasing $\mathrm{FEV}_{1}$ decline was associated with progressively greater morbidity and mortality risks. Groups with 'normal' lung function but accelerated declines had significantly increased mortality risks, including never smokers and individuals 45 years or younger. The results of these three studies using an at-risk population demonstrate the potential for COPD morbidity and mortality prevention through monitoring for lung function decline in males, females, never smokers, younger individuals, and those with 'normal' lung function. 


\section{DEDICATION}

I dedicate the completion of this dissertation to my family whose influences have helped me arrive at this point, but especially to my grandfather, Ralph Berton Shaver, whose curiosity inspired me as a child to wonder about the world as we "stomped around the farm" and whose suffering with chronic obstructive pulmonary disease inspired me as an adult to pursue this research in hopes that I might make a contribution that helps to prevent similar suffering for others. 


\section{ACKNOWLEDGEMENTS}

I thank Dr. Eva Hnizdo for her mentorship and for sharing her knowledge and expertise. Dr. Hnizdo set an example as a dedicated researcher and creative thinker, an outstanding scientist who crossed paths with a "country girl" from West Virginia with similar interests but with a need to acquire more knowledge and skills to explore them. Dr. Hnizdo provided that unique learning opportunity to me within the National Institute for Occupational Safety and Health (NIOSH).

I thank Dr. Michael E. Andrew, Dr. Christopher J. Martin, Dr. Edward L. Petsonk, and Dr. Anoop Shankar for their optimism and support as committee members. Each brought a unique contribution to my growth as a researcher. The group formed a very pleasant committee.

Many other people have helped along the way, including Community Medicine faculty whose doors were always open and staff who were always supportive and helpful. I thank our past and present PhD Program Directors, Dr. Robert Pack who encouraged me to apply for the program and Dr. Keith Zullig who helped me through to the end. I appreciate all of the experiences during the research rotations early in the program. The encouragement from Dr. Dina Jones, Dr. Jeffrey Coben, Dr. Robert Bossarte, Dr. Alan Ducatman, and Dr. Ian Rockett was reassuring as I transitioned into the role of a doctoral student. However, my journey began long before I enrolled in the $\mathrm{PhD}$ in Public Health Sciences Program. I thank those who over the years suggested that I consider doctoral education; it is unlikely that I would have pursued it without their encouragement. The most enthusiastic that I recall were Dr. L. Christopher Plein, Dr. Luc Anselin, Dr. M. Nawal Lutfiyya, Dr. Sandra Putnam, and especially Linda Sovine. With all of their input and that from Dr. Pack, I had enough data points to identify a trend and gave it a try.

I appreciate the chance to have worked in the NIOSH Division of Respiratory Disease Studies, Surveillance Branch during my dissertation research. It has been a special opportunity. NIOSH researchers were very helpful, offering suggestions about my research and thoughtfully inquiring about my progress, and staff warmly accommodated me as a guest researcher.

I thank the Copenhagen City Heart Study advisors for providing the data ultimately used for my dissertation research. I appreciate the kindness of Dr. Peter Lange and the support of Jacob L. Marott in using these data. I wish particularly to recognize the study participants, most of whom entered the study when I was a young child, oblivious of the future research opportunity that would be possible because of their willing participation. It has been an amazing opportunity.

I thank my colleagues in the program for not only celebrating my successes, but also helping me with challenges, mainly the "Ladies of the First Cohort": Ekta Choudhary, Danielle Davidov, Stephanie Frost, and Traci Jarrett; and Brent Doney at NIOSH. I am fortunate to know them all.

I appreciate the support provided by my family, who kindly listened to my ongoing tale as a doctoral student, and most of all to Aaron, husband and best friend, who waited patiently and supportively as I pursued yet another personal evolution in our life together. 


\section{TABLE OF CONTENTS}

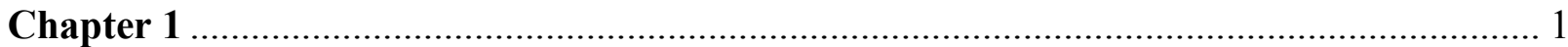

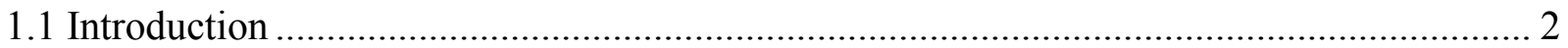

1.1.1 Incidence, Prevalence, and Costs of COPD .......................................................... 4

1.1.2 Contribution of Occupational Exposures to COPD .................................................. 5

1.1.3 Risk Factors for Lung Function Decline .................................................................... 6

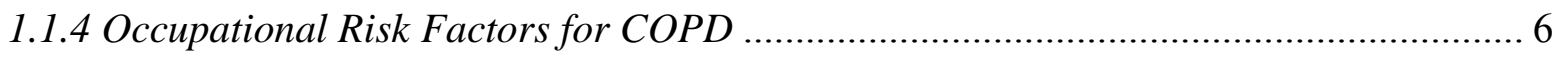

1.1.5 Longitudinal Studies of Lung Function Decline and Impairment .................................. 7

1.1.6 Occupational Longitudinal Studies of Lung Function Decline ..................................... 8

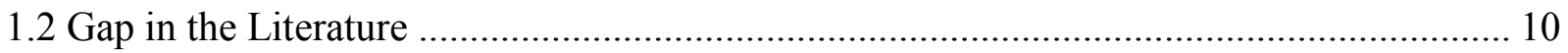

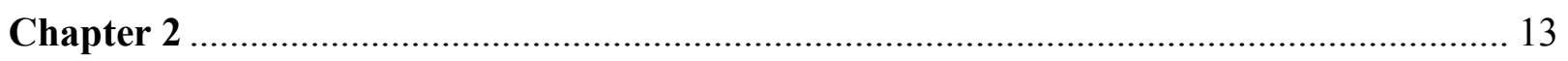

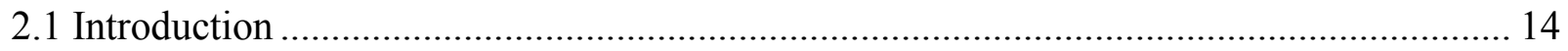

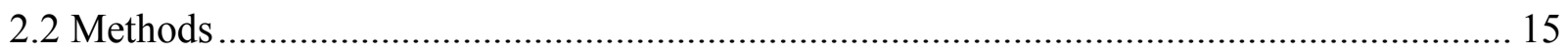

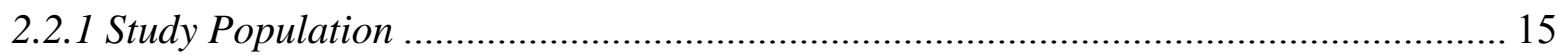

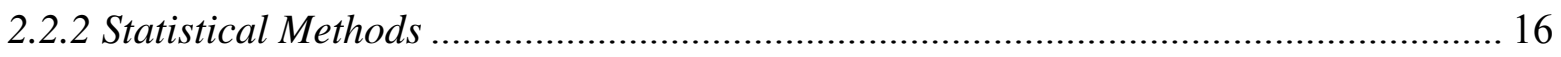

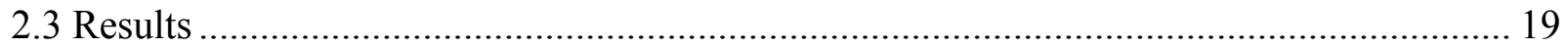

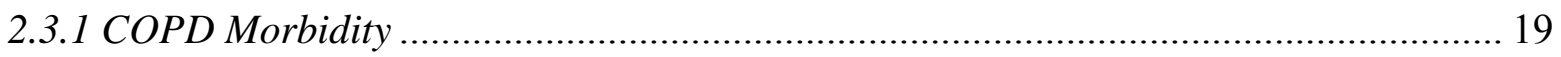

2.3.2 Mortality Due to COPD or CHD, and All-cause Mortality …………………............ 20

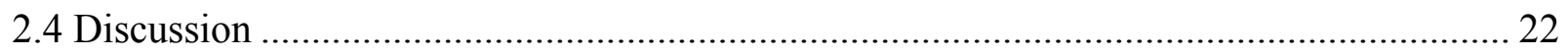

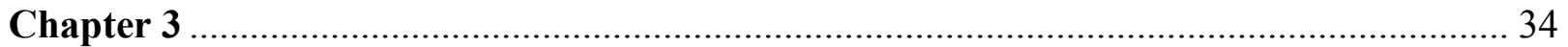

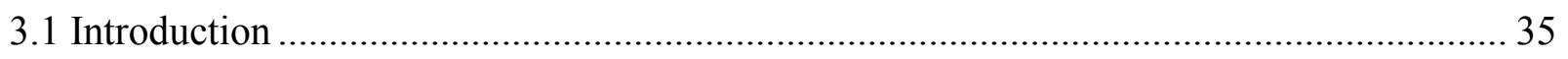

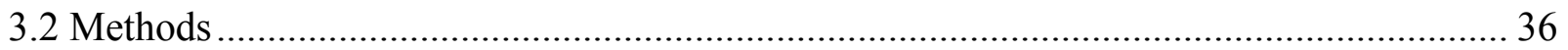

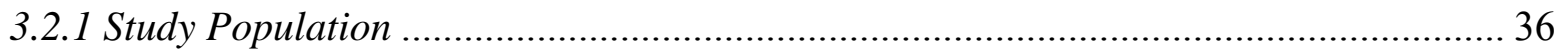

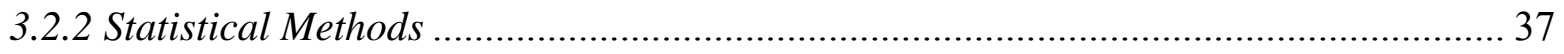

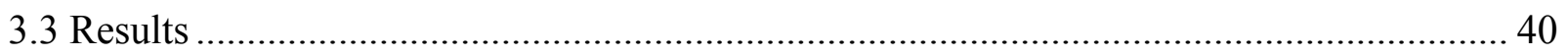

3.3.1 Overall Risks and Critical Cut-points in Lung Function Decline ................................ 40

3.3.2 Age-related Risks Associated with Lung Function Decline, Symptoms, and Asthma .. 41

3.3.3 Risks Associated with Temporal Patterns of Lung Function Decline and Symptoms.. 41

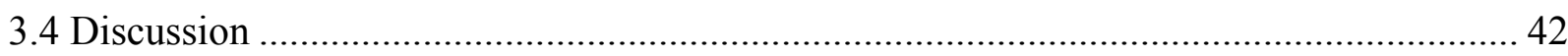




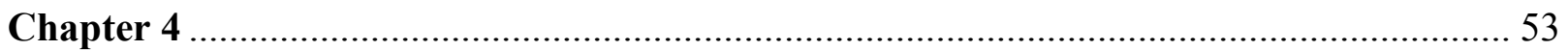

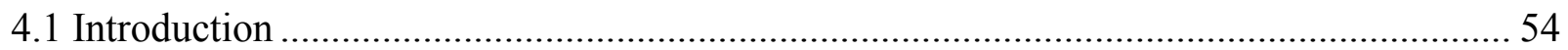

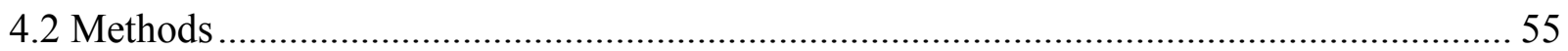

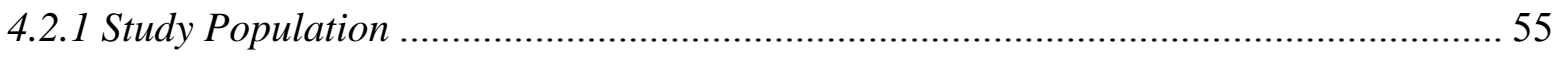

4.2.2 Statistical Methods ........................................................................................... 56

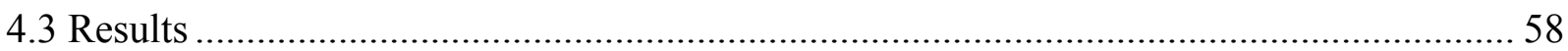

4.3.1 Overall Morbidity and Mortality Risks ............................................................... 58

4.3.2 Morbidity and Mortality Risks by Gender, for Never Smokers, and by Baseline Age

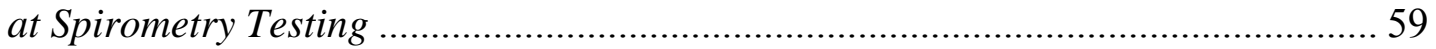

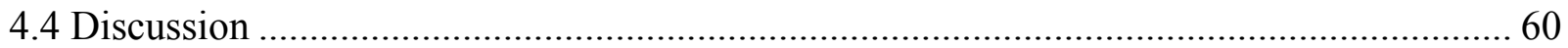

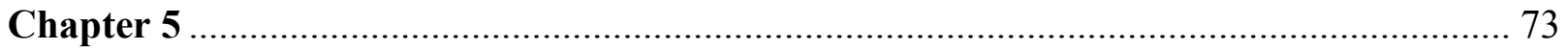

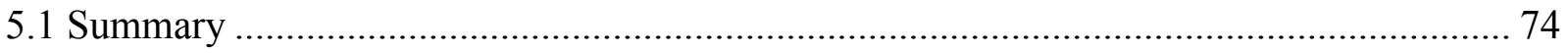

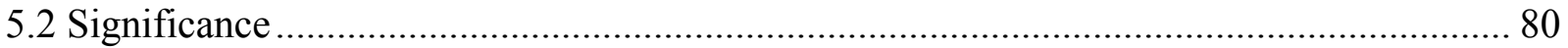

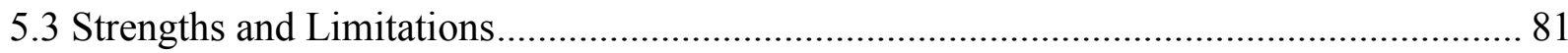

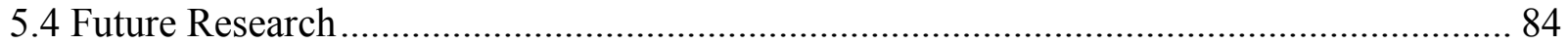

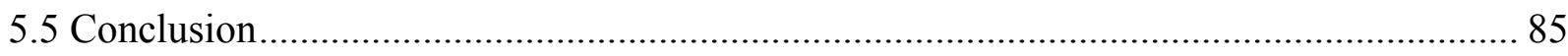

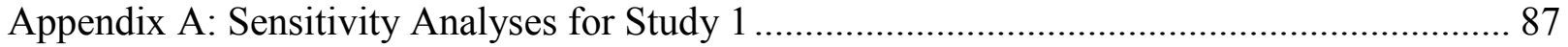

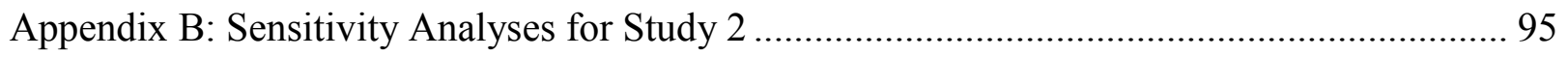

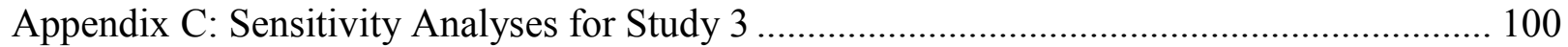

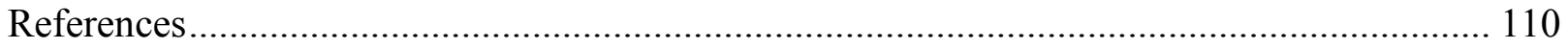


Chapter 1 


\section{Chapter 1}

\subsection{Introduction}

Excessive lung function decline is a marker of an underlying adverse health condition that may lead to the development of chronic obstructive pulmonary disease (COPD), the fourth leading cause of death in the United States and broadly defined as irreversible airflow limitation mainly caused by chronic bronchitis and emphysema, but asthma can in some situations also lead to COPD. The principal risk factor for COPD is tobacco smoking, but environmental factors, including occupational exposures also contribute to the development of COPD. ${ }^{1}$ Prevention of this disease due to occupational exposures is one of the major long-term goals of the National Institute for Occupational Safety and Health (NIOSH). ${ }^{2}$ The goal of this study is to evaluate the epidemiologic nature and utility of longitudinal measurements of forced expiratory volume in one second $\left(\mathrm{FEV}_{1}\right)$ as predictors for $\mathrm{COPD}, \mathrm{COPD}$ or coronary heart disease (CHD) mortality, and all-cause mortality in an aging, at-risk, population-based sample. Results of this evaluation will be applicable to community and workplace screening of at-risk individuals for respiratory disease and in the secondary prevention of disease.

COPD is generally a preventable disorder and involves progressive airflow limitation that is not fully reversible and includes chronic bronchitis, emphysema, and small airways disease. Asthma may also lead to irreversible airflow limitation identical to that of patients with COPD. Chronic bronchitis is defined clinically as a productive cough occurring on most days for at least three months during two consecutive years with no other identified causes for the symptoms. Emphysema is defined as permanent expansion of the airspaces distal to the terminal bronchioles and destruction of the walls in these airspaces, but without evident fibrosis. Small airways 
disease is associated with inflammatory processes in the lungs that lead to the deposition of collagen and thickening in the walls of the airways. ${ }^{1}$

Respiratory health monitoring can include periodic spirometry tests, administration of a respiratory symptom questionnaire, or radiological tests. ${ }^{3,4}$ Spirometry monitoring involves the evaluation of the level of lung function and the rate of lung function decline for the individual as well as for the group. For a group evaluation, differences in the level of lung function among groups can be estimated with a cross-sectional study design and multivariate linear regression analysis. The assessment of the influence of the rate of lung function decline on morbidity and mortality requires a longitudinal study and multivariate statistical methods that account for time to an event, such as Cox regression.

In the determination of whether an individual's lung function is within the normal limits, the level of lung function can be compared to reference values, based on the average level of lung function observed in healthy nonsmoking persons of the same age, sex, height, and race/ethnicity. When an individual's level of lung function falls below the lower limit of normal (LLN), the lung function level is considered abnormal. The LLN approximates the one-sided $95 \%$ confidence limit for the expected value, where $5 \%$ of apparently healthy individuals who have never smoked would be identified as abnormal. The methods for evaluation of the rate of decline in individuals in prospectively collected spirometry data as done in spirometry monitoring are not that well-established and are a subject of NIOSH current research. ${ }^{5,6}$ Questions remain about the usefulness of periodic spirometry and the screening criteria that would maximize its efficacy for disease prevention. 
In conducting research on spirometry monitoring, the longitudinal data collected in the Copenhagen City Heart Study were used to perform an epidemiological assessment of lung function decline as a predictor of adverse outcomes. The current study also investigated the relative effects of the level of lung function, the rate of decline, respiratory symptoms, and asthma as predictors of adverse outcomes. Results may provide answers to some of the remaining questions related to the development of guidance for effective intervention measures.

\subsubsection{Incidence, Prevalence, and Costs of COPD}

The World Health Organization estimates that worldwide 80 million people suffer moderate to severe COPD. COPD deaths in 2005 totaled more than 3 million people or $5 \%$ of all deaths, with nearly $90 \%$ of these COPD deaths occurring in middle and low-income countries. While COPD has been more common in males, the gender gap has been narrowing with increased tobacco use and exposure to biomass among females. As of 2002, COPD was the fifth leading cause of death. A $30 \%$ increase in COPD deaths in the following decade was projected without a reduction of the risk factors. By 2030, COPD is projected to be the third leading cause of death. ${ }^{7}$

Chronic lower respiratory diseases (International Classification of Diseases [ICD]-10 codes J40J47) were the fourth leading cause of death in the United States in 2005; COPD was the underlying cause of 126,005 deaths among adults age 25 or above. The crude death rate was 65.1 per 100,000 population and the age-adjusted death rate was 64.3 per 100,000 population. ${ }^{8}$ An estimated 10 million adults reported physician-diagnosed COPD in the United States in 2000, while the National Health and Nutrition Examination Survey (NHANES) III estimated that 24 million adults may have impaired lung function, suggesting that COPD is largely underdiagnosed. In 2000, COPD accounted for 8 million physician office and outpatient visits, 
1.5 million emergency department visits, and 726,000 hospitalizations. ${ }^{9}$ The estimated cost of COPD in the United States in 1998 was $\$ 26$ billion. ${ }^{10}$ Using NHANES III to model an adjusted relationship between COPD and labor force participation in the United States, people with COPD were $3.9 \%$ less likely to be in the labor force than those without the disease. The estimate of 1994 work loss due to COPD was $\$ 9.9$ billion in the United States. ${ }^{11}$

\subsubsection{Contribution of Occupational Exposures to COPD}

Recent population-based studies demonstrate the contribution of occupational exposures to COPD. Balmes' (2003) review indicates that approximately 15\% of asthma and COPD is likely to be related to occupational exposure. ${ }^{12}$ Leigh (2002) estimated 15,032 occupational COPD deaths in 1996 assuming a 15\% population attributable risk (PAR). Using data from the National Hospital Discharge Survey and the Healthcare Cost and Utilization Project Nationwide Inpatient Sample, the prevalence estimate was 2,395,650 for chronic bronchitis and emphysema combined (COPD) in 1996 due to occupational exposure. Related costs were estimated at $\$ 5.0$ billion. ${ }^{10}$

Based upon NHANES III data, the estimated attributable percent of COPD in the United States working population aged $30-75$ years was $19 \%$ overall and $31 \%$ for never smokers. ${ }^{13}$ In a 2003 study of older adults (55-75 years) who were surveyed about their health status, prior cigarette smoking, and occupational exposures, the estimated PAR for occupational exposure after adjusting for cigarette smoking was $20 \%$ for persons who reported a diagnosis of COPD, chronic bronchitis, or emphysema and $31 \%$ for persons who reported COPD or emphysema. ${ }^{14}$ Longitudinal studies estimate that after adjustment for age and smoking, the annual decline in $\mathrm{FEV}_{1}$ due to occupational exposures is about 7-9 milliliters per year ( $\left.\mathrm{ml} / \mathrm{yr}\right)$ and the effect of dust exposure may be greater than that of cigarette smoking alone for heavily exposed workers. ${ }^{12}$ 


\subsubsection{Risk Factors for Lung Function Decline}

While risk factors are not the focus of this study, they define the populations at risk for accelerated lung function decline and that may benefit from screening and intervention activities. The American Thoracic Society/European Respiratory Society task force emphasized a number of host factors (genetic, gender, airway hyperreactivity, immunoglobulin E, and asthma) and exposures (smoking, socioeconomic status, occupation, environmental pollution, perinatal events and childhood illness, recurrent bronchopulmonary infections, and diet) that place individuals at

risk for COPD. ${ }^{1}$ Additional host factors that can increase the risk of lung function decline include preexisting lung diseases such as tuberculosis or pneumonia and abdominal adiposity. ${ }^{15}$ This wide range of risk factors, to which a great number of individuals are exposed, helps explain the importance of lung function decline as a public health problem that is reflected in COPD as a leading cause of mortality.

\subsubsection{Occupational Risk Factors for COPD}

Reviews over the years reveal the increasing knowledge on the many occupational risk factors including coal mining, gold mining, uranium mining, pyrite mining, foundry work, steel work, furnace and chemical work, textile manufacturing, grain and vegetable-product work, and toluene diisocyanate work that are among the risky occupations. ${ }^{16}$ Airflow limitation due to dusty occupations lead to research on the relationships of acute and chronic disease relative to many airborne pollutants. ${ }^{17}$ Evidence on the risks of dust and dust and fumes was gathered from various types of studies ${ }^{18}$ and occupational dust was identified as a cause of chronic bronchitis, lung function decline, and increased risk of COPD in smokers. ${ }^{19}$ 
Hendrick et. al. surmised that occupation can exert a COPD effect that varies by industry, level of exposure, and substance; that interactions with smoking are probable; that multiple environmental agents may cause COPD; and that COPD would be infrequent if both smoking and asthma were absent. ${ }^{20}$ Dimich-Ward et. al. identified an association between inorganic dusts and chronic airflow limitation, separate from pneumoconiosis. ${ }^{21}$ Coggon and Newman Taylor concluded that there was strong evidence of lung function impairment and disability from coal mine dust exposure, and an additive effect between coal mine dust and smoking on $\mathrm{FEV}_{1}{ }^{22}$ Evidence on the association between COPD and silica dust exposure suggested that chronic long-term exposure can lead to COPD, that the association between cumulative exposure and airflow obstruction was independent of silicosis, and that smoking augments the effect of silica dust on airflow obstruction and COPD deaths. ${ }^{23}$ The association between COPD and occupational exposures has been found in hard-rock miners, tunnel workers, concretemanufacturing workers, and in non-mining industrial workers. Epidemiologic studies demonstrate a relationship between dust exposure and the degree of emphysema in coal and hard-rock miners. ${ }^{12}$

\subsubsection{Longitudinal Studies of Lung Function Decline and Impairment}

Several studies have examined $\mathrm{FEV}_{1}$ decline or impairment as a predictor of morbidity and mortality. The risk of all-cause mortality has been frequently investigated, ${ }^{24-34}$ but risks for mortality due to cardiovascular and coronary heart disease, ${ }^{28,35-38}$ cancer, lung cancer, stroke, and respiratory disease ${ }^{28,36}$ have also been explored. There are fewer studies of the risks for COPD incidence and hospitalizations. ${ }^{33,34,39}$ In these prior studies, the study designs focused upon identifying lung function decline as an independent risk factor. Models were adjusted for a range of other risk factors, as appropriate to the outcome under study, including age, sex, race, 
height, weight, body mass index, baseline $\mathrm{FEV}_{1}$, average $\mathrm{FEV}_{1}$, shortness of breath on exertion, attacks of shortness of breath, smoking and/or pack-years, years since smoking cessation, occupational exposures to dust and to variable heat, hypertension, diabetes, coronary heart disease, cardiovascular disease risk factors, cholesterol levels, depression, blood group, number of infants delivered, alcohol intake, physical activity level, education, and social class.

As these previous studies have demonstrated ample statistically significant associations between lung function decline and the risks of morbidity and mortality while adjusting for other risk factors, the current study uses these results as a basis to investigate lung function decline as a predictor of morbidity and mortality in minimally-adjusted models. These models were intended to be informative for prevention practices based on observed lung function decline. Models in the current study were adjusted for age, height, and baseline lung function and stratified by gender, conducted for never smokers, and stratified by baseline age at spirometry testing to consider major biological factors determining lung function and the principal risk factor for COPD, tobacco smoking, that was highly prevalent in the study sample.

\subsubsection{Occupational Longitudinal Studies of Lung Function Decline}

In an early study of French industrial workers with 12 years of follow-up for men aged 30 to 54, the individual annual rate of decline was estimated from measurements in 1960 and 1972. The $\mathrm{FEV}_{1}$ slope was significantly related to exposure to mineral dust and grain dust, with a doseresponse relationship, and to heat exposure. Among those heavily exposed, slopes for workers who changed jobs fared better than those who did not. Across various exposures to dust, gas, and

heat, average slopes ranged from $44-60 \mathrm{ml} / \mathrm{yr}$ after adjusting for age, smoking, and $\mathrm{FEV}_{1}$ level. $^{40}$ Data from the United States National Coal Study demonstrated losses in $\mathrm{FEV}_{1}$ related to 
smoking (0.1 liter over 11 years) and occupational exposure (0.036-0.084 liter over 11 years), confirming findings in a study of British coal workers. ${ }^{41}$

A Swedish study of mortality in 300,000 construction workers identified a significantly higher risk of COPD mortality for workers exposed to fumes and mineral dust. ${ }^{42}$ Among paper pulp workers, the relationship between ozone exposure and lung function was examined. Increased risks for declines in $\mathrm{FEV}_{1}$ and forced vital capacity (FVC) were found with reported chlorine dioxide/sulphur dioxide gassings. ${ }^{43}$ In a study of the effects of exposure to silicon metal and alloys through smelting on lung function, line operators working in the ferrosilicon/silicon metal and silicon carbide smelters had sharper annual lung function decline in comparison to nonexposed workers. ${ }^{44}$ The effect of ongoing fume and dust exposure was studied on the rate of progression of COPD for individuals in its early stages. Fume exposure was associated with reduced post-bronchodilator $\mathrm{FEV}_{1}$ percent predicted in males, but was not associated with the rate of decline in prebronchodilator $\mathrm{FEV}_{1}$. There was a nonstatistically significant trend in the association of dust exposure with lower prebronchodilator $\mathrm{FEV}_{1}$ in females. This suggests a sensitivity to fume exposure and that occupational exposure to fume in individuals with COPD adversely affects lung function. ${ }^{45} \mathrm{~A}$ study of the effects of smoking and occupational exposure on COPD identified higher incidence and prevalence of COPD in smokers with occupational exposures to dusts, fumes, and vapors after 5 and 10 years as compared to workers with one or neither exposure, and an interaction was identified between the exposures. ${ }^{46}$

Respiratory symptoms and reduced lung function were associated with an increased dropout rate in a longitudinal study of Norwegian smelters. The most recently reported symptoms were more predictive of dropout than those reported at baseline; shortness of breath was the most important 
symptom. ${ }^{47}$ A study on the relationship between lung function and occupational dust exposure in smelter workers demonstrated that annual change was negatively associated with increasing exposure. $^{48}$

\subsection{Gap in the Literature}

Although spirometry monitoring is recommended in workplaces where workers are exposed to respiratory hazards, there are very few recommendations on methods for using spirometry results in the prevention of respiratory disease. Since the mid 1970s, various spirometry monitoring programs have been implemented and longitudinal spirometry data are now available to help develop such methods and recommendations. Further examination of longitudinal spirometry data is needed, including a comparison of questionnaire-reported symptoms versus spirometry to provide a scientific basis for the development of recommendations to enhance community and workplace screening of at-risk individuals in the prevention of respiratory disease. The objective of the current study relates to three Healthy People 2020 Respiratory Disease, Chronic Obstructive Pulmonary Disease objectives. ${ }^{49}$

- RD-10: Reduce deaths from chronic obstructive pulmonary disease (COPD) among adults.

- $\mathrm{RD}-11$ : Reduce hospitalizations for chronic obstructive pulmonary disease (COPD).

- RD-13: (Developmental) Increase the proportion of adults with abnormal lung function whose underlying obstructive disease has been diagnosed.

The objective also generally relates to a goals of the National Institute for Occupational Safety and Health to prevent and reduce work-related COPD. ${ }^{2}$ Importantly, decreasing rates of smoking 
in the working population will result in increasing significance of occupational exposures causing $\mathrm{COPD}^{50}$ and greater need for a more precise understanding of the occupational contribution to COPD and how to best recognize future disease using prospectively collected data.

The studies in Chapters 2-4 are secondary data analyses of the Copenhagen City Heart Study longitudinal spirometry data and self-reported respiratory symptom and asthma data. These data were used to estimate risks of COPD, COPD or CHD mortality, and all-cause mortality in a population-based sample at risk for these outcomes, given the high prevalence of smoking in both males and females. The study in Chapter 2 examines the relative contributions of lung function decline and respiratory symptoms and asthma in predicting these outcomes with particular relevance to workplace respiratory disease prevention programs, where longitudinal spirometry and respiratory symptom questionnaires are primary tools used to identify respiratory disease. This study also compares morbidity and mortality risks associated with multiple measures of longitudinal lung function decline: the $\mathrm{FEV}_{1}$ slope; the $\mathrm{FEV}_{1}$ relative slope; the American College of Occupational and Environmental Medicine's (ACOEM) Longitudinal Normal Limit (LNL); and a $90 \mathrm{ml} / \mathrm{yr}$ limit. ${ }^{51,52}$ The study in Chapter 3 evaluates two issues not fully explored in the literature: 1) associations of lung function decline and respiratory symptoms and asthma with morbidity and mortality in individuals younger than middle age (when COPD typically becomes evident); and 2) associations with long-term patterns of decline and symptoms. The study in Chapter 4 uses a novel approach to assess the combined effect of the baseline level of lung function and subsequent lung function decline on morbidity and mortality risks to help further identify high-risk groups. 
In summary, there is limited research using minimally-adjusted models to explore a more direct relationship of lung function decline as a predictor of morbidity and mortality. Earlier studies focused on estimating the adjusted relationship of lung function decline to morbidity and mortality, to establish it as an independent risk factor. Our studies adjusted only for essential covariates that would typically be available and taken into consideration in a clinical context ${ }^{53}$ and build upon the existing evidence of increased risks associated with lung function decline. These minimally-adjusted models permit a different evaluation of lung function decline, one with clinical implications for prevention and intervention based on observed lung function decline.

These studies advance scientific knowledge by offering estimates of morbidity and mortality risks due to lung function decline, and also the level of lung function, and self-reported respiratory symptoms and asthma in a large, at-risk, aging, population-based sample. Estimates were identified in males, females, never smokers, individuals above and below middle age, and those with a 'normal' level of lung function, but excessive lung function decline. The associations identified in this study may be useful for health care providers who evaluate lung function decline in patients. This research increases knowledge relevant to the assessment of longitudinal lung function decline and to the integration of respiratory symptom questionnaire data into the decision-making process on prevention and intervention strategies. These studies strengthen the rationale and broaden the scientific basis for the development of recommendations to enhance screening for respiratory disease. 
Chapter 2 


\section{Chapter 2}

\subsection{Introduction}

Studies of various populations demonstrate that lung function can predict adverse health outcomes, such COPD morbidity and mortality, CHD mortality, and all-cause mortality. ${ }^{24-34,39,}$ ${ }^{54-57}$ COPD is a costly disease, with high prevalence rates in the United States and internationally, high associated healthcare costs, early retirement from work, and excess premature mortality. COPD is also associated with CHD, another costly disease. ${ }^{7,10,11,36,58-61}$ While the principal risk factor for COPD is tobacco smoking, occupational exposures also contribute to the development of COPD and its cost. ${ }^{12-14,36,45,46,62-65}$ Industry-based studies identify increased healthcare utilization and disability associated with COPD, and demonstrate the need for prevention of excessive loss of lung function. ${ }^{66,67}$ In workers with potential exposure to occupational respiratory hazards, effective monitoring of longitudinal lung function is one approach to early recognition of lung function impairment and provides opportunities for developing prevention strategies to reduce potentially modifiable risk factors associated with long-term adverse health outcomes.

Periodic lung function evaluation can support effective prevention strategies in the workplace. Important issues to consider are: the critical rate at which lung function decline becomes a significant predictor of morbidity and mortality; the measures of lung function decline that best predict morbidity and mortality; and the role of respiratory symptoms and asthma in the prediction. Specific cut-points in the $\mathrm{FEV}_{1}$ rate of decline were considered in a previous study of mortality in coal miners. ${ }^{68}$ This study showed increased mortality risk with rates of decline above 
about $60 \mathrm{ml} / \mathrm{yr}$ in never smokers and with declines of $90 \mathrm{ml} / \mathrm{yr}$ in all miners; furthermore, the decline of $60 \mathrm{ml} / \mathrm{yr}$ or more was associated with increased disability and early retirement. ${ }^{36}$

The aim of this study was to evaluate the risks of COPD morbidity based on hospitalizations, COPD or CHD mortality, and all-cause mortality associated with excessive lung function decline, current respiratory symptoms of chronic bronchitis and shortness of breath, and current asthma in an aging community cohort. Specific objectives were to evaluate the usefulness of various measures of lung function decline in predicting morbidity and mortality, to investigate the critical rate of lung function decline that becomes a significant predictor of morbidity and mortality, and to assess the usefulness of current respiratory symptoms and asthma in predicting morbidity and mortality relative to lung function level and decline.

\subsection{Methods}

\subsubsection{Study Population}

Our study used data from the Copenhagen City Heart Study, a prospective study of cardiovascular disease in 23,000 males and females aged 20 years and older. ${ }^{69,70}$ The study involved four clinical examinations (including spirometry) and a self-administered questionnaire conducted over a 28-year period (1976-78, 1981-83, 1991-94, and 2001-03). A minimum of three spirometry tests were performed at each session, in a sitting position, using an electronic spirometer (N 403 Monaghan, United States). At least two measurements had to be within 5\% of

one another. ${ }^{69,70}$ For analysis of lung function decline, we selected subjects who had spirometry tests at examinations one and two, approximately five years apart. Change in $\mathrm{FEV}_{1}$ was evaluated because it is the most useful measurement for assessing the rate of lung function decline. ${ }^{71}$ Height measurements were collected as part of the physical examination. Age and 
gender data were obtained from the Copenhagen Population Register at the time of study enrollment. Smoking status was self-reported at each examination.

Current respiratory symptoms of chronic bronchitis and current asthma were self-reported at examinations one and two, while shortness of breath was initially ascertained at examination two. In preliminary univariate analyses, we identified asthma ("Do you suffer from asthma?") ${ }^{72}$ chronic bronchitis ("Do you bring up phlegm, in the morning or during the day, for as long as three months each year?"), and shortness of breath ("Do you often feel out of breath?") as the best predictors of future morbidity and mortality.

Morbidity data were from the National Patient Register, mortality data from the Civil Registration System, and causes of death from the National Register of Causes of Death. Three health outcomes were studied: 1) primary and secondary hospital diagnoses of COPD morbidity (ICD-8 491-492, and ICD-10 J41-J44); 2) COPD or CHD mortality (ICD-8 410-414, and ICD10 I20-I25) as an underlying or contributing cause; and 3) all-cause mortality. (Denmark transitioned directly from the $8^{\text {th }}$ to the $10^{\text {th }}$ revision of ICD in 1993.) COPD morbidity follow-up was until 12/31/2003. Mortality follow-up was until 12/31/2006 for cause-specific mortality, and until 8/11/2007 for all causes.

\subsubsection{Statistical Methods}

The Cox proportional hazards model was used to estimate the hazard (instantaneous event rate) of morbidity or mortality in relation to lung function level and decline, respiratory symptoms, and asthma, while adjusting for essential covariates that would typically be available and taken into consideration in a clinical context. ${ }^{53}$ Analyses were conducted by gender and for never 
smokers. Additional Cox models using penalized splines were conducted for the overall cohort to identify the rate of $\mathrm{FEV}_{1}$ slope at which morbidity and mortality risks began to increase. For COPD morbidity, time to event (or censor) was calculated from examination two until COPD diagnosis, death, or end of follow-up. For mortality, time to event (or censor) was calculated from examination two until death or end of follow-up.

First, we evaluated the relationships of the level of lung function measurements by examination (one or two) with morbidity and mortality. Models included quartiles of lung function level $\left(\mathrm{FEV}_{1} /\right.$ height $\left.^{2}\right){ }^{73}$ respiratory symptoms, asthma, and age. The highest lung function level $\left(75^{\text {th }}\right.$ percentile and above) served as the reference category. As the results were generally similar, only examination two results are presented because shortness of breath was often statistically significant $(p<0.05)$ and was unavailable at examination one.

Second, we evaluated the relationship of the rate of lung function decline, and respiratory symptoms and asthma ascertained at examination two with morbidity and mortality, adjusted for baseline age, height-adjusted baseline lung function $\left(\mathrm{FEV}_{1} /\right.$ height $\left.^{2}\right)$, and height (Figure 2.1). In separate analyses, we evaluated four measures of lung function decline: 1) the $\mathrm{FEV}_{1}$ slope (the difference in $\mathrm{FEV}_{1}$ between examinations one and two, divided by the time between the examinations) in quartiles; 2) the $\mathrm{FEV}_{1}$ relative slope (slope divided by baseline $\mathrm{FEV}_{1}$ ) in quartiles; 3) the American College of Occupational and Environmental Medicine's (ACOEM) Longitudinal Normal Limit (LNL) decline based on the American Thoracic Society's (ATS) $15 \%$ annual limit and an expected decline of $30 \mathrm{ml} / \mathrm{yr} ;{ }^{51}$ and 4) a $\mathrm{FEV}_{1}$ decline of $90 \mathrm{ml} / \mathrm{yr}$ or more. ${ }^{52}$ The ACOEM LNL is designed to identify excessive declines during early years of spirometry follow-up (1-8 years) when data are sparse and a reliable slope cannot be estimated. ${ }^{51}$ 
To identify trends in risk, we categorized the slopes into quartiles and used the quartiles with the lowest rates of decline ( $75^{\text {th }}$ percentile and above) as the reference categories. The risks associated with excessive lung function decline, defined by the two longitudinal limits (LNL and a decline of $90 \mathrm{ml} / \mathrm{yr}$ or more), were compared to subjects with 'normal' decline. The two limits were compared for predicting risk, and for model fit using the Akaike's information criterion (AIC).$^{74}$

Preliminary analysis of mean $\mathrm{FEV}_{1}$ values by year of examination revealed a slightly excessive increase in mean $\mathrm{FEV}_{1}$ in $1981(\mathrm{n}=4,319)$, but not in $1982(\mathrm{n}=5,353)$ or $1983(\mathrm{n}=2,593)$, as compared to mean $\mathrm{FEV}_{1}$ for baseline examinations in 1976-78. It is unknown to the authors whether this increase in 1981 was due to early difficulties with the Monaghan spirometer that was replaced with a dry wedge spirometer by examination three or to other issues. ${ }^{70}$ To adjust for this increase, we reduced the individual $\mathrm{FEV}_{1}$ measurements for 1981 by a fixed value of $289 \mathrm{ml}$ for males and $201 \mathrm{ml}$ for females to align the 1981 values with the 1976-78 and 1982-83 values. The fixed values represent the average difference in $\mathrm{FEV}_{1}$ values from examination one to two (for 1982 only), minus $30 \mathrm{ml}$ to correct for annual loss from 1981 to 1982 . Models excluding the 1981 values were conducted for a sensitivity analysis regarding this adjustment.

Copenhagen City Heart Study participants gave informed consent to participate and the study was performed in accordance with the $2^{\text {nd }}$ Helsinki Declaration and approved by the Danish regional ethics committee. The present study was approved by the National Institute for Occupational Safety and Health Human Subjects Review Board and the West Virginia University Institutional Review Board. Analyses were conducted with PROC PHREG $(p<0.05)$ 
in SAS, version 9.2 (SAS Institute Inc, USA). Penalized splines were prepared in R software, version 2.7.2.

\subsection{Results}

Three subcohorts (males, females, and never smokers) used in the Cox models are described in Table 2.1. The table shows the duration of follow-up for morbidity and mortality (15-20 years), the mean and quartile values for $\mathrm{FEV}_{1} /$ height $^{2}$ (level of lung function) and the two types of slopes (lung function decline), and the frequency of symptoms and asthma. For never smokers, we presented gender-specific quartiles because females represented $75 \%$ of the never-smoker subcohort. (Sample sizes for the analysis of lung function decline were smaller because members had to participate at both examinations one and two.) Baseline characteristics for subjects who were lost to follow-up by examination two were a higher average age, lower average $\mathrm{FEV}_{1} /$ height $^{2}$, and a higher percentage of self-reported current asthma, symptoms of chronic bronchitis, and smoking.

\subsubsection{COPD Morbidity}

Estimated hazard ratios (HR) for COPD morbidity (hospital diagnosis of COPD) are presented in Table 2.2. The level of lung function $\left(\mathrm{FEV}_{1} /\right.$ height $\left.^{2}\right)$ was a significant predictor of COPD morbidity for males and females starting from the second quartile. A similar, but less significant trend was observed in never smokers, where significance was observed only at the fourth quartile.

A similar increasing trend in COPD morbidity risk was seen for the $\mathrm{FEV}_{1}$ slope, except significance began at the third quartile for never smokers. Females had the highest HRs for 
COPD morbidity for the slope, the relative slope, the LNL, and the $90 \mathrm{ml} / \mathrm{yr}$ limit (Figure 2.2a). Results from the penalized spline analysis showed that the log HR for COPD morbidity (males and females combined) started to increase at an $\mathrm{FEV}_{1}$ slope of $-54 \mathrm{ml} / \mathrm{yr}$ (Figure 2.3a). The parameter for the linear rate was highly significant $(p<0.0001)$.

Comparing results for the dichotomous outcomes, the LNL was significantly associated with COPD morbidity for all three subcohorts, as was the $90 \mathrm{ml} / \mathrm{yr}$ limit. When compared using the AIC ${ }^{74}$ the fit of these two models were similar. The LNL limit provided a slightly better fit for females, but the $90 \mathrm{ml} / \mathrm{yr}$ limit was slightly better for males and never smokers.

Respiratory symptoms and asthma were often significantly associated with COPD morbidity in the models with the level of lung function and lung function decline. The risks associated with chronic bronchitis and shortness of breath were higher than for asthma for males and females. In never smokers, the risk associated with asthma was the highest with a four-fold increase.

\subsubsection{Mortality Due to COPD or CHD, and All-cause Mortality}

For mortality outcomes, there was significant association with decreasing level of lung function for all three subcohorts (Tables 2.3 and 2.4). The risk of COPD or CHD mortality in relation to the level of lung function was slightly higher than for all-cause mortality, with females and never smokers often having higher risks than males. For COPD or CHD mortality, females in the fourth quartile had more than a five-fold increase in risk, never smokers a nearly four-fold increase in risk, and males a three-fold increase in risk as compared to the reference. Hazard ratios for all-cause mortality were more similar for the three subcohorts. 
Increased risk of mortality began at the second quartile of the $\mathrm{FEV}_{1}$ slope and relative slope for females, and most often at the third quartile for males and the fourth quartile for never smokers. Figures $2.3 \mathrm{~b}$ and $2.3 \mathrm{c}$ show the penalized splines for the slope for the mortality outcomes in males and females combined. The nonlinear terms were significant $(p=0.01$ for COPD or CHD mortality and $p=0.0001$ for all-causes) and the spline crossed above zero for the $\log \mathrm{HR}$ at -72 $\mathrm{ml} / \mathrm{yr}$ for COPD or CHD mortality and at $-77 \mathrm{ml} / \mathrm{yr}$ for all-cause mortality.

As for the dichotomous criteria (the LNL and the $90 \mathrm{ml} / \mathrm{yr}$ limit), there were significant associations with both mortality outcomes, with the exception of the LNL and COPD or CHD mortality in never smokers. The AIC values for these two criteria were similar by subcohort and outcome, but the models with the LNL fit best overall, with the exceptions of COPD or CHD mortality in males and in never smokers. A comparison of all of the results by subcohort demonstrates that females had the highest HRs for both mortality outcomes for all four measures of lung function decline.

Asthma was not significantly associated with increased risk of mortality. Chronic bronchitis was significantly associated with mortality, but not for never smokers; COPD or CHD mortality was approximately $30 \%$ more likely for males and $60 \%$ for females who reported chronic bronchitis. In the models with the level of lung function, shortness of breath was significantly associated with mortality for all three subcohorts; doubling the risk of COPD or CHD mortality for males and females, and increasing the risk of all-cause mortality by $50 \%$. Similar results were observed in the models with lung function decline. 


\subsection{Discussion}

Unlike studies concerned with identifying morbidity and mortality risk factors, our interest was examining the relationship of respiratory status (lung function, respiratory symptoms, and asthma) with morbidity and mortality. This study relates to clinical practice and prevention as it focuses on the predictive, rather than causative, nature of these respiratory factors. The level of lung function was associated with high risks for morbidity and mortality, supporting the need for prevention. For example, in at-risk occupational populations, monitoring lung function over time can be used to identify individuals at risk of lung function impairment and initiate appropriate intervention. Our results add to current knowledge on lung function decline as a predictor of morbidity and mortality in aging populations.

\subsubsection{Evaluating the Usefulness of the Measures of Lung Function Decline}

The results demonstrate the predictive capacities of the four criteria for lung function decline (the $\mathrm{FEV}_{1}$ slope and relative slope, the ACOEM LNL based on the ATS 15\% year-to-year limit, and a $\mathrm{FEV}_{1}$ decline of $90 \mathrm{ml} / \mathrm{yr}$ or more) for morbidity and mortality. ${ }^{51,52}$ Associations were stronger with COPD morbidity, as might be expected, given its identification using a hospital diagnosis which would focus on greater disease severity (Figure 2.2a). Also as expected, associations with mortality due to COPD or CHD were slightly higher than with all-cause mortality (Figures $2.2 \mathrm{~b}$ and 2.2c); possibly due to a more direct relationship of COPD and CHD mortality with lung function. Females were at greater risk of morbidity and mortality than males across all measures of lung function decline. This may be partially explained by smaller lung capacity in females; loss of lung function could pose more risk. ${ }^{75}$ Also, given that females in this study had lower tobacco consumption than males, this may suggest higher susceptibility in females. Other studies also find females more susceptible to the effects of smoking and with more rapid decline than 
males, and possibly with greater propensity for the development of COPD. ${ }^{76,77}$ HRs for never smokers showed an increasing trend for all outcomes, but less statistical significance (Tables $2.2-2.4)$

Among the four criteria, quartiles for the slope had higher HRs, but goodness of fit (AIC) was generally better for the relative slope. The slightly lower risk and better fit for the relative slope are reasonable given the adjustment for baseline lung function level. As for the two limits (the LNL and the $90 \mathrm{ml} / \mathrm{yr}$ limit), the HRs were similar, but often slightly higher for the LNL, and the goodness of fit was often better for the LNL.

\subsubsection{Investigating Critical Rates of Lung Function Decline}

HRs for COPD morbidity suggest a critical rate at the third quartile of the slope with risks greater than 2.5 times those for the reference (Figure 2.2a), corresponding to declines starting at $60 \mathrm{ml} / \mathrm{yr}$ for males and $57 \mathrm{ml} / \mathrm{yr}$ for females (Table 2.1). For COPD or CHD mortality, the critical rate is more likely at the fourth quartile of the slope, with risks nearly doubled for males and tripled for females (Figure 2.2b). For all-cause mortality, fourth quartile risks were increased by $67 \%$ and $88 \%$ for males and females, respectively (Figure $2.2 \mathrm{c}$ ), corresponding to declines starting at $118 \mathrm{ml} / \mathrm{yr}$ for males and $95 \mathrm{ml} / \mathrm{yr}$ for females (Table 2.1).

Our results generally agree with several community-based and occupational studies of lung function decline and respiratory morbidity and mortality. ${ }^{29,33,36,57,68}$ Similarities include slightly higher increased risks in females of COPD hospitalization and all-cause mortality associated with rapid decline as compared to males ${ }^{29,33}$ and increased risk of cardiac mortality with

increasing decline in males. ${ }^{57}$ Also similar to our results, underground coal miners had a two-fold 
risk of cardiovascular and nonmalignant respiratory disease mortality with rapid decline, ${ }^{36}$ and declines above $90 \mathrm{ml} / \mathrm{yr}$ were statistically significant for mortality as were declines of $60 \mathrm{ml} / \mathrm{yr}$ or more in never smokers. ${ }^{68}$

\subsubsection{Assessing the Usefulness of Respiratory Symptoms and Asthma Relative to Lung Function}

Risks associated with lung function decline were often greater than with respiratory symptoms and asthma, though their risks remained statistically significant after adjustment for lung function. Asthma predicted COPD morbidity in males and never smokers. Chronic bronchitis and shortness of breath predicted morbidity and mortality in males and females, with higher HRs in females for chronic bronchitis which could relate to increased susceptibility to the effects of respiratory hazards. ${ }^{77}$

Other studies have also reported increased risks of morbidity and mortality in relation to reported respiratory symptoms (chronic bronchitis, cough, phlegm, and shortness of breath) and asthma, ${ }^{78-}$ ${ }^{82}$ even after controlling for $\mathrm{FEV}_{1} \cdot{ }^{83}$ In contrast, we did not find increased risks between asthma and respiratory mortality; however, our study targeted COPD specifically. ${ }^{78}$ Chronic bronchitis often remained a significant predictor of mortality in our study, even with adjustment for shortness of breath and height-adjusted baseline lung function. ${ }^{83}$

There are several limitations including the necessary adjustment in the $\mathrm{FEV}_{1}$ values collected in 1981. Therefore, influences of the adjusted data were investigated by excluding it from the analysis, and significant overestimation of the HRs was not identified. Rather, the inclusion of the adjusted data resulted in an underestimation of the HRs. In particular, COPD morbidity HRs were underestimated for the level of lung function, the slope, and the relative slope, but mainly 
for never smokers and more often for females than for males. There was also underestimation of the HRs for COPD or CHD mortality for the level of lung function for never smokers.

There are other possible limitations related to lung function decline. One is the use of only two spirometry measurements for the rate of change in $\mathrm{FEV}_{1}$, leaving the slope vulnerable to instability in either measurement, but the quartile groupings could have reduced this effect. The limit of $90 \mathrm{ml} / \mathrm{yr}$ or more was from literature pertaining to males. ${ }^{52}$ Although females generally have smaller lungs ${ }^{75}$ results using this criterion were similar to those for the LNL and the fourth quartile of the slope for females, a corresponding decline of $95 \mathrm{ml} / \mathrm{yr}$ or more. In studying lung function decline, we did not account for possible mixing between cause and effect with regard to steeply declining lung function and COPD outcomes, but our interest was in prediction rather than causation.

Underdiagnosis of COPD and underreporting of COPD as a cause of death could have resulted in misclassification, biasing the results toward the null, and reducing the associations. ${ }^{84}$ This was addressed by combining the often interrelated COPD and CHD mortality into one outcome..$^{60,61}$ As COPD morbidity was limited to a hospital diagnosis, our results generally represent risks for individuals with more severe disease, but also likely the highest costs. Other possible selection bias includes subjects lost to follow-up by examination two who were older at baseline, had a lower lung function level, and a higher proportion of self-reported current asthma, chronic bronchitis, and smoking as compared those who participated at examinations one and two. This could have resulted in an underestimation of risk.

Our findings are relevant to clinical and workplace disease prevention and cost-reduction. ${ }^{66,67}$ Investment in health promotion and protection for COPD could create savings for employers. ${ }^{85}$, 
${ }^{86}$ The strong associations of COPD morbidity with lung function level and decline correspond with high medical costs for COPD and indicate that effective monitoring and interpretation of lung function decline for early disease detection could lead to cost-savings.

In conclusion, the risks of COPD morbidity, COPD or CHD mortality, and all-cause mortality showed an increasing trend with lung function decline after adjustment for baseline lung function, respiratory symptoms, and asthma. The level and rate of lung function decline generally demonstrated higher risks of morbidity and mortality than the respiratory symptoms and asthma. These results provide further evidence that evaluation of lung function decline, in addition to the lung function level, is important in spirometry monitoring programs. 


\begin{tabular}{ll}
$\begin{array}{l}\text { Examination 1 } \\
1976-78\end{array}$ & $\begin{array}{c}\text { Examination } 2 \\
1981-83 \\
13,831 \text { Subjects }\end{array}$ \\
\hline $\begin{array}{l}\text { Study cohort: } \\
9,679 \text { Subjects }\end{array}$ & $\begin{array}{l}\text { Spirometry and ascertainment } \\
\text { of asthma, chronic bronchitis, } \\
\text { and shortness of breath }\end{array}$ \\
\hline
\end{tabular}

Figure 2.1 Sample size and morbidity and mortality follow-up. Above the timeline are the numbers of subjects who participated in spirometry testing by Copenhagen City Heart Study examination. Below the timeline is the number of subjects who participated in spirometry testing at examinations one and two and the numbers of health outcomes that occurred during the morbidity and mortality follow-up periods. Note that the follow-up periods are represented as dashed lines. COPD morbidity follow-up lasted until 2003. COPD or CHD mortality follow-up lasted until 2006. All-cause mortality follow-up lasted until 2007. 


\begin{tabular}{|c|c|c|c|c|c|c|}
\hline \multirow{2}{*}{$\begin{array}{l}\text { Data for lung function level } \\
\text { (Examination two) } \\
\text { Subjects }(\mathrm{n}, \%)^{*}\end{array}$} & \multicolumn{2}{|c|}{ Males } & \multicolumn{2}{|c|}{ Females } & \multicolumn{2}{|c|}{ Never smokers } \\
\hline & 5,494 & 44.8 & 6,771 & 55.2 & 2,555 & 20.8 \\
\hline Age at examination two (mean, $\mathrm{SD} \dagger$ ) & 56.1 & 12.6 & 56.7 & 12.1 & 56.4 & 13.8 \\
\hline \multicolumn{7}{|l|}{ Follow-up, years (mean, SD) } \\
\hline COPD morbidity & 15.4 & 6.9 & 17.3 & 6.0 & 18.1 & 5.7 \\
\hline All-cause mortality & 17.1 & 8.1 & 19.5 & 7.2 & 20.3 & 7.0 \\
\hline Height, $\mathrm{cm} \dagger$ (mean, SD) & 174 & 7 & 161 & 6 & 164 & 9 \\
\hline $\mathrm{FEV}_{1}, 1 \dagger$ (mean, $\left.\mathrm{SD}\right)$ & 2.82 & 0.91 & 2.05 & 0.63 & 2.39 & 0.85 \\
\hline $\mathrm{FEV}_{1} /$ height $^{2} \mathrm{x}$ mean height ${ }^{2}, 1$ (mean, SD) & 2.57 & 0.75 & 2.17 & 0.60 & 2.42 & 0.67 \\
\hline 25th percentile & 2.07 & & 1.78 & & $2.43 ; 1.87 \ddagger$ & \\
\hline Chronic bronchitis (n, \%) & 872 & 15.9 & 722 & 10.7 & 148 & 5.8 \\
\hline Shortness of breath (n, \%) & 543 & 9.9 & 728 & 10.8 & 159 & 6.2 \\
\hline Never smokers (n, \%) & 629 & 11.5 & 1,926 & 28.5 & $629 ; 1,926 \div$ & $24.6 ; 75.4 \ddagger$ \\
\hline Former smokers (n, \%) & 1,352 & 24.6 & 1,244 & 18.4 & & \\
\hline Current smokers (n, \%) & 3,510 & 63.9 & 3,592 & 53.1 & & \\
\hline \multicolumn{7}{|l|}{$\begin{array}{l}\text { Data for lung function decline } \\
\text { (Examination one to two) } \S\end{array}$} \\
\hline Subjects $(\mathrm{n}, \%)^{*}$ & 4,253 & 43.9 & 5,426 & 56.1 & 1,822 & 18.8 \\
\hline Baseline age (mean, SD) & 52.5 & 11.4 & 52.7 & 10.8 & 52.8 & 12.2 \\
\hline \multicolumn{7}{|l|}{ Follow-up, years (mean, SD) } \\
\hline 25th percentile & -118 & & -95 & & $-114 ;-94 \$$ & \\
\hline Median & -60 & & -57 & & $-57 ;-40 \ddagger$ & \\
\hline 75th percentile & 0 & & 0 & & $19 ; 0 \%$ & \\
\hline Slope $\mathrm{FEV}_{1} /$ baseline $\mathrm{FEV}_{1}, \% / \mathrm{yr}$ (mean, SD) & -2.0 & 3.4 & -1.9 & 3.4 & -1.5 & 3.4 \\
\hline 25th percentile & -4.1 & & -4.1 & & $-3.2 ;-3.8 \ddagger$ & \\
\hline Median & -2.2 & & -2.2 & & $-1.5 ;-2.0 \ddagger$ & \\
\hline 75th percentile & 0.0 & & 0.0 & & $0.4 ; 0.0 \ddagger$ & \\
\hline $\mathrm{FEV}_{1}$ below $\mathrm{LNL} \dagger(\mathrm{n}, \%)$ & 743 & 17.4 & 1,016 & 18.7 & 346 & 19.0 \\
\hline $\mathrm{FEV}_{1}$ decline of $90 \mathrm{ml} / \mathrm{yr}$ or more $(\mathrm{n}, \%)$ & 1,717 & 40.4 & 1,444 & 26.6 & 505 & 27.7 \\
\hline Asthma (n, \%) & 125 & 2.9 & 168 & 3.1 & 40 & 2.2 \\
\hline Chronic bronchitis (n, \%) & 665 & 15.6 & 558 & 10.3 & 96 & 5.3 \\
\hline Shortness of breath (n, \%) & 401 & 9.4 & 550 & 10.1 & 104 & 5.7 \\
\hline
\end{tabular}

*A total of 12,265 subjects at examination two and 9,679 subjects at examinations one and two. $\dagger$ SD, standard deviation; cm, centimeters; 1, liters; ml/yr, milliliters/year; LNL, Longitudinal Normal Limit. †ales; females. $\S$ Includes subjects with approximately five years between spirometry tests. 


\begin{tabular}{|c|c|c|c|c|c|c|}
\hline \multirow{3}{*}{$\begin{array}{l}\text { Data for lung function level* } \\
\text { (Examination two) }\end{array}$} & \multicolumn{2}{|c|}{ Males } & \multirow{2}{*}{\multicolumn{2}{|c|}{$\begin{array}{c}\text { Females } \\
(\mathrm{n}=6,735 ; \text { COPD }=678)\end{array}$}} & \multirow{2}{*}{\multicolumn{2}{|c|}{$\begin{array}{c}\text { Never smokers } \\
(\mathrm{n}=2,548 ; \mathrm{COPD}=73)\end{array}$}} \\
\hline & \multicolumn{2}{|c|}{$(\mathrm{n}=5,442 ; \mathrm{COPD}=563)$} & & & & \\
\hline & HR & $95 \%$ CI & HR & $95 \% \mathrm{CI}$ & HR & \multirow[t]{2}{*}{$95 \% \mathrm{Cl}$} \\
\hline $\mathrm{Q} 1 \mathrm{FEV}_{1} /$ height $^{2}$ & \multicolumn{2}{|l|}{1.00} & \multicolumn{2}{|l|}{1.00} & 1.00 & \\
\hline Q2 $\mathrm{FEV}_{1} /$ height $^{2}$ & 2.69 & $(1.73-4.18)$ & 2.66 & $(1.85-3.83)$ & 1.64 & $(0.55-4.87)$ \\
\hline Q3 $\mathrm{FEV}_{1} /$ height $^{2}$ & 6.10 & $(3.99-9.33)$ & 5.09 & $(3.57-7.25)$ & 1.41 & $(0.46-4.34)$ \\
\hline Q4 FEV ${ }_{1} /$ height $^{2}$ & 16.36 & $(10.67-25.07)$ & 16.55 & $(11.65-23.53)$ & 5.18 & $(1.81-14.80)$ \\
\hline Asthma $\dagger$ & 2.21 & $(1.66-2.93)$ & 1.20 & $(0.91-1.58)$ & 3.69 & $(1.67-8.15)$ \\
\hline Chronic bronchitis $\dagger$ & 2.04 & $(1.68-2.47)$ & 2.05 & $(1.70-2.48)$ & 2.35 & $(1.26-4.38)$ \\
\hline Shortness of breath $\dagger$ & 2.48 & $(2.00-3.08)$ & 2.77 & $(2.29-3.36)$ & 2.36 & $(1.21-4.58)$ \\
\hline Data for lung function decline & \multicolumn{2}{|c|}{$(\mathrm{n}=4,214 ; \mathrm{COPD}=442)$} & \multicolumn{2}{|c|}{$(\mathrm{n}=5,402 ; \mathrm{COPD}=537)$} & \multicolumn{2}{|c|}{$(\mathrm{n}=1,817 ; \mathrm{COPD}=49)$} \\
\hline (Examination one to two) & HR & $95 \% \mathrm{CI}$ & HR & $95 \% \mathrm{CI}$ & HR & $95 \% \mathrm{CI}$ \\
\hline Q1 FEV Flope & 1.00 & & 1.00 & & 1.00 & \\
\hline Q2 $\mathrm{FEV}_{1}$ slope & 1.61 & $(1.18-2.19)$ & 2.48 & $(1.89-3.27)$ & 1.80 & $(0.73-4.45)$ \\
\hline Q3 $\mathrm{FEV}_{1}$ slope & 2.54 & $(1.87-3.45)$ & 3.37 & $(2.55-4.44)$ & 2.53 & $(1.01-6.33)$ \\
\hline Q4 FEV ${ }_{1}$ slope & 3.77 & $(2.76-5.15)$ & 6.12 & $(4.63-8.10)$ & 3.58 & $(1.34-9.62)$ \\
\hline Asthma & 1.41 & $(1.00-1.99)$ & 0.80 & $(0.58-1.12)$ & 4.45 & $(1.40-14.11)$ \\
\hline Chronic bronchitis & 1.92 & $(1.53-2.40)$ & 2.24 & $(1.82-2.77)$ & 2.00 & $(0.84-4.79)$ \\
\hline Shortness of breath & 2.00 & $(1.54-2.61)$ & 2.22 & $(1.76-2.78)$ & 0.69 & $(0.21-2.22)$ \\
\hline Q1 $\mathrm{FEV}_{1}$ relative slope & 1.00 & & 1.00 & & 1.00 & \\
\hline $\mathrm{Q}_{2} \mathrm{FEV}_{1}$ relative slope & 1.50 & $(1.09-2.07)$ & 1.99 & $(1.48-2.68)$ & 1.73 & $(0.68-4.41)$ \\
\hline Q3 $\mathrm{FEV}_{1}$ relative slope & 2.17 & $(1.58-3.00)$ & 2.60 & $(1.95-3.46)$ & 1.65 & $(0.60-4.57)$ \\
\hline $\mathrm{Q}_{4} \mathrm{FEV}_{1}$ relative slope & 3.48 & $(2.59-4.67)$ & 5.27 & $(4.06-6.85)$ & 3.92 & $(1.63-9.44)$ \\
\hline Asthma & 1.32 & $(0.93-1.86)$ & 0.80 & $(0.57-1.11)$ & 4.39 & $(1.38-13.92)$ \\
\hline Chronic bronchitis & 1.95 & $(1.56-2.44)$ & 2.26 & $(1.82-2.79)$ & 2.03 & $(0.87-4.73)$ \\
\hline Shortness of breath & 2.04 & $(1.57-2.65)$ & 2.21 & $(1.76-2.78)$ & 0.69 & $(0.22-2.18)$ \\
\hline $\mathrm{FEV}_{1}$ below LNL & 2.32 & $(1.86-2.88)$ & 3.01 & $(2.49-3.64)$ & 2.33 & $(1.22-4.44)$ \\
\hline Asthma & 1.52 & $(1.08-2.14)$ & 0.86 & $(0.62-1.20)$ & 4.49 & $(1.46-13.78)$ \\
\hline Chronic bronchitis & 1.98 & $(1.58-2.48)$ & 2.37 & $(1.91-2.93)$ & 2.18 & $(0.93-5.10)$ \\
\hline Shortness of breath & 2.10 & $(1.62-2.74)$ & 2.27 & $(1.81-2.86)$ & 0.76 & $(0.24-2.39)$ \\
\hline $\mathrm{FEV}_{1}$ decline of $90 \mathrm{ml} / \mathrm{yr}$ or more & 2.11 & $(1.73-2.57)$ & 2.87 & $(2.37-3.48)$ & 2.32 & $(1.19-4.53)$ \\
\hline Asthma & 1.48 & $(1.05-2.09)$ & 0.82 & $(0.59-1.15)$ & 4.74 & $(1.56-14.46)$ \\
\hline Chronic bronchitis & 2.02 & $(1.61-2.52)$ & 2.34 & $(1.89-2.89)$ & 1.97 & $(0.81-4.80)$ \\
\hline Shortness of breath & 2.12 & $(1.63-2.75)$ & 2.38 & $(1.90-2.98)$ & 0.78 & $(0.25-2.41)$ \\
\hline
\end{tabular}

*Models adjusted for age at examination two.

$\dagger$ Asthma, chronic bronchitis, and shortness of breath represented as dichotomous variables in all models.

$\$$ Models adjusted for baseline age, height-adjusted baseline lung function $\left(\mathrm{FEV}_{1} /\right.$ height $^{2}$ ), and height. See Table 2.1 for quartile values.

$\mathrm{FEV}_{1}$ relative slope, slope $\mathrm{FEV}_{1}$ /baseline $\mathrm{FEV}_{1}$; LNL, Longitudinal Normal Limit. 


\begin{tabular}{|c|c|c|c|c|c|c|}
\hline \multirow{3}{*}{$\begin{array}{l}\text { Data for lung function level* } \\
\text { (Examination two) }\end{array}$} & \multirow{2}{*}{\multicolumn{2}{|c|}{$\begin{array}{c}\text { Males } \\
(\mathrm{n}=5,494 ; \text { Deaths }=1,201)\end{array}$}} & \multirow{2}{*}{\multicolumn{2}{|c|}{$\begin{array}{c}\text { Females } \\
(\mathrm{n}=6,771 ; \text { Deaths }=983)\end{array}$}} & \multirow{2}{*}{\multicolumn{2}{|c|}{$\begin{array}{c}\text { Never smokers } \\
(\mathrm{n}=2,555 ; \text { Deaths }=269)\end{array}$}} \\
\hline & & & & & & \\
\hline & HR & $95 \% \mathrm{CI}$ & HR & $95 \% \mathrm{CI}$ & HR & $95 \%$ CI \\
\hline Q1 $\mathrm{FEV}_{1} /$ height $^{2}$ & \multicolumn{2}{|l|}{1.00} & \multicolumn{2}{|l|}{1.00} & \multicolumn{2}{|l|}{1.00} \\
\hline Q2 $\mathrm{FEV}_{1} /$ height $^{2}$ & 1.46 & $(1.15-1.85)$ & 1.88 & $(1.38-2.57)$ & 1.92 & $(0.95-3.89)$ \\
\hline Q3 $\mathrm{FEV}_{1} /$ height $^{2}$ & 2.02 & $(1.60-2.56)$ & 2.79 & $(2.06-3.77)$ & 2.54 & $(1.29-5.01)$ \\
\hline Q4 FEV $1 /$ height $^{2}$ & 3.02 & $(2.38-3.84)$ & 5.49 & $(4.07-7.41)$ & 3.94 & $(2.00-7.76)$ \\
\hline Asthma † & 1.14 & $(0.88-1.47)$ & 0.87 & $(0.66-1.15)$ & 1.03 & $(0.49-2.14)$ \\
\hline Chronic bronchitis $\dagger$ & 1.32 & $(1.14-1.53)$ & 1.62 & $(1.37-1.93)$ & 1.32 & $(0.88-1.99)$ \\
\hline Shortness of breath $\dagger$ & 2.15 & $(1.82-2.54)$ & 2.30 & $(1.94-2.72)$ & 1.57 & $(1.04-2.35)$ \\
\hline Data for lung function declinet & \multicolumn{2}{|c|}{$(\mathrm{n}=4,253 ;$ Deaths $=974)$} & \multicolumn{2}{|c|}{$(\mathrm{n}=5,426 ;$ Deaths $=796)$} & \multicolumn{2}{|c|}{$(\mathrm{n}=1,822 ;$ Deaths $=202)$} \\
\hline (Examination one to two) & HR & $95 \%$ CI & HR & $95 \%$ CI & HR & $95 \% \mathrm{CI}$ \\
\hline Q1 FEV 1 slope & 1.00 & & 1.00 & & 1.00 & \\
\hline Q2 $\mathrm{FEV}_{1}$ slope & 1.20 & $(0.99-1.45)$ & 1.48 & $(1.19-1.83)$ & 1.17 & $(0.77-1.78)$ \\
\hline Q3 $\mathrm{FEV}_{1}$ slope & 1.34 & $(1.10-1.63)$ & 1.73 & $(1.39-2.15)$ & 1.42 & $(0.93-2.17)$ \\
\hline Q4 FEV ${ }_{1}$ slope & 1.91 & $(1.57-2.33)$ & 2.95 & $(2.38-3.67)$ & 1.42 & $(0.90-2.25)$ \\
\hline Asthma & 0.94 & $(0.70-1.27)$ & 0.74 & $(0.54-1.03)$ & 1.30 & $(0.56-3.03)$ \\
\hline Chronic bronchitis & 1.29 & $(1.10-1.52)$ & 1.58 & $(1.31-1.92)$ & 1.33 & $(0.80-2.22)$ \\
\hline Shortness of breath & 1.92 & $(1.58-2.33)$ & 1.86 & $(1.52-2.28)$ & 1.21 & $(0.70-2.09)$ \\
\hline Q1 FEV 1 relative slope & 1.00 & & 1.00 & & 1.00 & \\
\hline $\mathrm{Q}^{2} \mathrm{FEV}_{1}$ relative slope & 1.15 & $(0.94-1.40)$ & 1.51 & $(1.21-1.90)$ & 1.08 & $(0.69-1.67)$ \\
\hline Q3 $\mathrm{FEV}_{1}$ relative slope & 1.31 & $(1.07-1.60)$ & 1.27 & $(1.00-1.60)$ & 1.15 & $(0.74-1.80)$ \\
\hline $\mathrm{Q} \mathrm{FEV}_{1}$ relative slope & 1.75 & $(1.45-2.11)$ & 2.68 & $(2.19-3.28)$ & 1.61 & $(1.06-2.43)$ \\
\hline Asthma & 0.90 & $(0.67-1.22)$ & 0.68 & $(0.49-0.94)$ & 1.31 & $(0.56-3.04)$ \\
\hline Chronic bronchitis & 1.29 & $(1.10-1.53)$ & 1.60 & $(1.32-1.95)$ & 1.32 & $(0.79-2.19)$ \\
\hline Shortness of breath & 1.95 & $(1.61-2.37)$ & 1.90 & $(1.56-2.33)$ & 1.17 & $(0.68-2.02)$ \\
\hline $\mathrm{FEV}_{1}$ below LNL & 1.56 & $(1.34-1.82)$ & 2.14 & $(1.84-2.50)$ & 1.34 & $(0.97-1.85)$ \\
\hline Asthma & 0.95 & $(0.70-1.28)$ & 0.73 & $(0.52-1.01)$ & 1.27 & $(0.55-2.95)$ \\
\hline Chronic bronchitis & 1.31 & $(1.11-1.54)$ & 1.63 & $(1.34-1.98)$ & 1.32 & $(0.79-2.21)$ \\
\hline Shortness of breath & 1.95 & $(1.60-2.36)$ & 1.86 & $(1.51-2.27)$ & 1.22 & $(0.71-2.10)$ \\
\hline $\mathrm{FEV}_{1}$ decline of $90 \mathrm{ml} / \mathrm{yr}$ or more & 1.54 & $(1.35-1.75)$ & 2.12 & $(1.81-2.48)$ & 1.43 & $(1.03-1.99)$ \\
\hline Asthma & 0.94 & $(0.70-1.27)$ & 0.76 & $(0.55-1.05)$ & 1.33 & $(0.57-3.08)$ \\
\hline Chronic bronchitis & 1.30 & $(1.11-1.54)$ & 1.62 & $(1.33-1.96)$ & 1.31 & $(0.78-2.18)$ \\
\hline Shortness of breath & 1.98 & $(1.63-2.39)$ & 1.89 & $(1.55-2.31)$ & 1.19 & $(0.69-2.05)$ \\
\hline
\end{tabular}

*Models adjusted for age at examination two.

$\dagger$ Asthma, chronic bronchitis, and shortness of breath represented as dichotomous variables in all models.

$\$$ Models adjusted for baseline age, height-adjusted baseline lung function $\left(\mathrm{FEV}_{1} /\right.$ height $\left.^{2}\right)$, and height. See Table 2.1 for quartile values.

$\mathrm{FEV}_{1}$ relative slope, slope $\mathrm{FEV}_{1}$ /baseline $\mathrm{FEV}_{1}$; LNL, Longitudinal Normal Limit. 


\begin{tabular}{|c|c|c|c|c|c|c|}
\hline \multirow{3}{*}{$\begin{array}{l}\text { Data for lung function level* } \\
\text { (Examination two })\end{array}$} & \multicolumn{2}{|c|}{ Males } & \multirow{2}{*}{\multicolumn{2}{|c|}{$\begin{array}{c}\text { Females } \\
(\mathrm{n}=6,771 ; \text { Deaths }=3,632)\end{array}$}} & \multirow{2}{*}{\multicolumn{2}{|c|}{$\begin{array}{c}\text { Never smokers } \\
(\mathrm{n}=2,555 ; \text { Deaths }=1,181)\end{array}$}} \\
\hline & \multicolumn{2}{|c|}{$(\mathrm{n}=5,494 ;$ Deaths $=3,535)$} & & & & \\
\hline & HR & $95 \% \mathrm{CI}$ & HR & $95 \% \mathrm{CI}$ & HR & $95 \% \mathrm{CI}$ \\
\hline Q1 $\mathrm{FEV}_{1} /$ height $^{2}$ & \multicolumn{2}{|l|}{1.00} & \multicolumn{2}{|l|}{1.00} & \multicolumn{2}{|l|}{1.00} \\
\hline Q2 $\mathrm{FEV}_{1} /$ height $^{2}$ & 1.27 & $(1.13-1.44)$ & 1.39 & $(1.22-1.57)$ & 1.54 & $(1.20-1.98)$ \\
\hline Q3 $\mathrm{FEV}_{1} /$ height $^{2}$ & 1.60 & $(1.42-1.81)$ & 1.83 & $(1.62-2.07)$ & 1.70 & $(1.33-2.16)$ \\
\hline Q4 FEV $1 /$ height $^{2}$ & 2.31 & $(2.04-2.62)$ & 2.65 & $(2.33-3.01)$ & 2.15 & $(1.68-2.76)$ \\
\hline Asthma $\dagger$ & 0.98 & $(0.82-1.17)$ & 0.99 & $(0.83-1.18)$ & 1.13 & $(0.78-1.63)$ \\
\hline Chronic bronchitis $\dagger$ & 1.29 & $(1.18-1.40)$ & 1.32 & $(1.20-1.46)$ & 1.16 & $(0.94-1.44)$ \\
\hline Shortness of breath $\dagger$ & 1.52 & $(1.36-1.70)$ & 1.50 & $(1.36-1.67)$ & 1.38 & $(1.11-1.70)$ \\
\hline Data for lung function decline $\ddagger$ & \multicolumn{2}{|c|}{$(\mathrm{n}=4,253 ;$ Deaths $=2,842)$} & \multicolumn{2}{|c|}{$(\mathrm{n}=5,426 ;$ Deaths $=2,958)$} & \multicolumn{2}{|c|}{$(\mathrm{n}=1,822 ;$ Deaths $=860)$} \\
\hline (Examination one to two) & HR & $95 \% \mathrm{CI}$ & HR & $95 \% \mathrm{CI}$ & HR & $95 \% \mathrm{CI}$ \\
\hline Q1 FEV Flope & 1.00 & & 1.00 & & 1.00 & \\
\hline Q2 $\mathrm{FEV}_{1}$ slope & 1.14 & $(1.02-1.27)$ & 1.24 & $(1.12-1.39)$ & 1.17 & $(0.95-1.43)$ \\
\hline Q3 $\mathrm{FEV}_{1}$ slope & 1.20 & $(1.08-1.35)$ & 1.37 & $(1.23-1.53)$ & 1.31 & $(1.06-1.61)$ \\
\hline Q4 $\mathrm{FEV}_{1}$ slope & 1.67 & $(1.49-1.87)$ & 1.88 & $(1.68-2.10)$ & 1.53 & $(1.23-1.91)$ \\
\hline Asthma & 0.87 & $(0.71-1.07)$ & 0.96 & $(0.78-1.16)$ & 1.15 & $(0.73-1.80)$ \\
\hline Chronic bronchitis & 1.24 & $(1.12-1.38)$ & 1.27 & $(1.14-1.43)$ & 1.10 & $(0.84-1.45)$ \\
\hline Shortness of breath & 1.43 & $(1.26-1.63)$ & 1.36 & $(1.21-1.54)$ & 1.27 & $(0.97-1.66)$ \\
\hline Q1 $\mathrm{FEV}_{1}$ relative slope & 1.00 & & 1.00 & & 1.00 & \\
\hline Q2 $\mathrm{FEV}_{1}$ relative slope & 1.10 & $(0.98-1.23)$ & 1.25 & $(1.11-1.39)$ & 1.18 & $(0.96-1.46)$ \\
\hline Q3 $\mathrm{FEV}_{1}$ relative slope & 1.23 & $(1.09-1.37)$ & 1.22 & $(1.09-1.37)$ & 1.13 & $(0.91-1.40)$ \\
\hline $\mathrm{Q} 4 \mathrm{FEV}_{1}$ relative slope & 1.55 & $(1.39-1.73)$ & 1.81 & $(1.63-2.01)$ & 1.59 & $(1.29-1.95)$ \\
\hline Asthma & 0.84 & $(0.68-1.04)$ & 0.91 & $(0.74-1.10)$ & 1.11 & $(0.71-1.74)$ \\
\hline Chronic bronchitis & 1.24 & $(1.12-1.37)$ & 1.28 & $(1.15-1.44)$ & 1.09 & $(0.83-1.44)$ \\
\hline Shortness of breath & 1.45 & $(1.28-1.65)$ & 1.38 & $(1.22-1.55)$ & 1.26 & $(0.97-1.65)$ \\
\hline $\mathrm{FEV}_{1}$ below LNL & 1.48 & $(1.35-1.62)$ & 1.58 & $(1.46-1.72)$ & 1.42 & $(1.22-1.66)$ \\
\hline Asthma & 0.87 & $(0.71-1.07)$ & 0.94 & $(0.77-1.15)$ & 1.11 & $(0.71-1.74)$ \\
\hline Chronic bronchitis & 1.25 & $(1.13-1.39)$ & 1.30 & $(1.16-1.45)$ & 1.11 & $(0.84-1.46)$ \\
\hline Shortness of breath & 1.44 & $(1.27-1.64)$ & 1.36 & $(1.21-1.54)$ & 1.29 & $(0.99-1.69)$ \\
\hline $\mathrm{FEV}_{1}$ decline of $90 \mathrm{ml} / \mathrm{yr}$ or more & 1.37 & $(1.27-1.48)$ & 1.55 & $(1.42-1.68)$ & 1.37 & $(1.17-1.61)$ \\
\hline Asthma & 0.87 & $(0.70-1.07)$ & 0.97 & $(0.80-1.18)$ & 1.14 & $(0.73-1.79)$ \\
\hline Chronic bronchitis & 1.25 & $(1.13-1.39)$ & 1.29 & $(1.15-1.44)$ & 1.10 & $(0.83-1.45)$ \\
\hline Shortness of breath & 1.47 & $(1.30-1.67)$ & 1.37 & $(1.22-1.55)$ & 1.29 & $(0.99-1.68)$ \\
\hline
\end{tabular}

*Models adjusted for age at examination two.

$\dagger$ Asthma, chronic bronchitis, and shortness of breath represented as dichotomous variables in all models.

$\ddagger$ Models adjusted for baseline age, height-adjusted baseline lung function $\left(\mathrm{FEV}_{1} /\right.$ height $\left.^{2}\right)$, and height. See Table 2.1 for quartile values.

$\mathrm{FEV}_{1}$ relative slope, slope $\mathrm{FEV}_{1}$ /baseline $\mathrm{FEV}_{1}$; LNL, Longitudinal Normal Limit. 

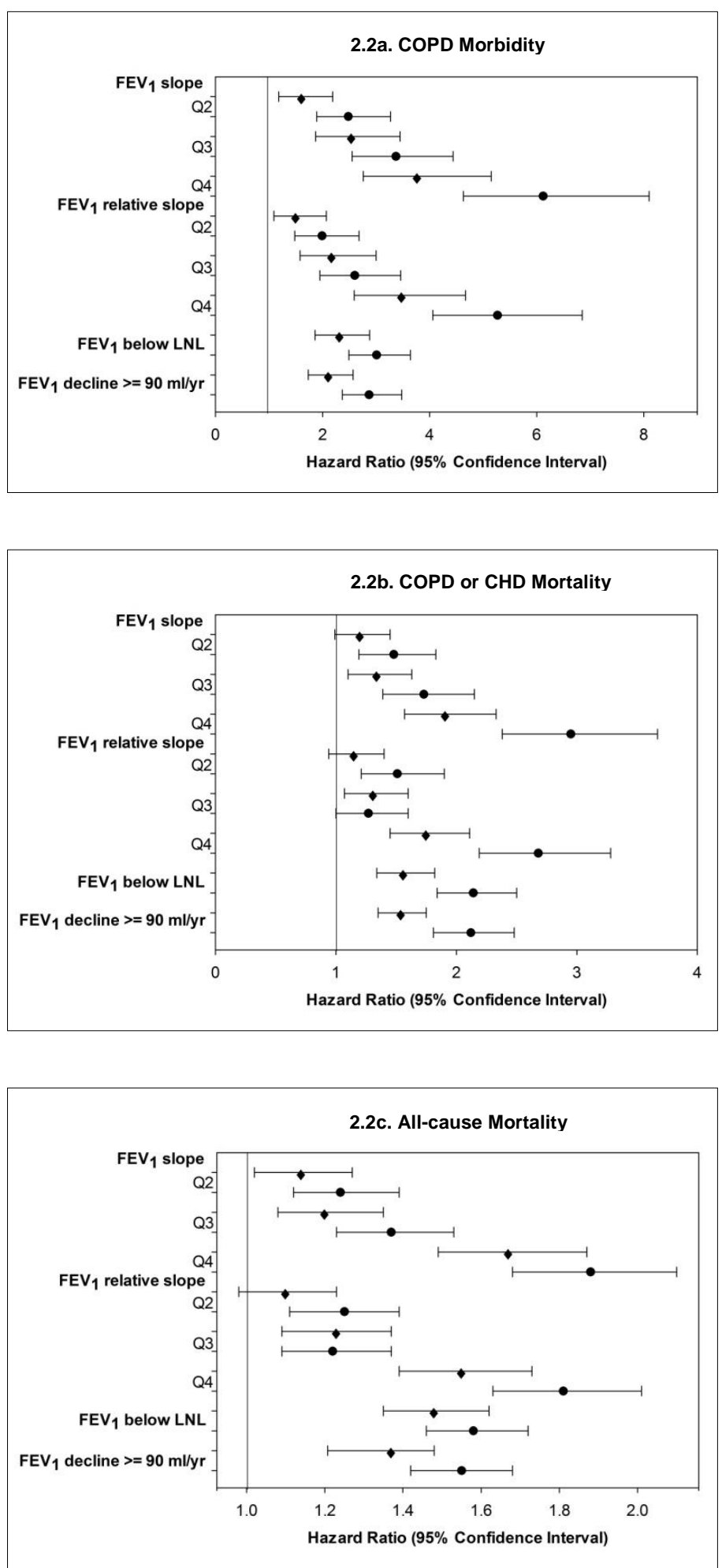

Figure 2.2 Cox proportional hazards model results by gender $(\bullet=$ males and $\bullet=$ females) for (a) COPD morbidity, (b) COPD or CHD mortality, and (c) all-cause mortality. Models adjusted for baseline age, height-adjusted baseline lung function ( $F E V_{1} /$ height $\left.^{2}\right)$, height, and respiratory symptoms and asthma as dichotomous variables. See Table 2.1 for quartile values. FEV ${ }_{1}$ relative slope, slope FEV /baseline FEV $_{1}$; LNL, Longitudinal Normal Limit. 

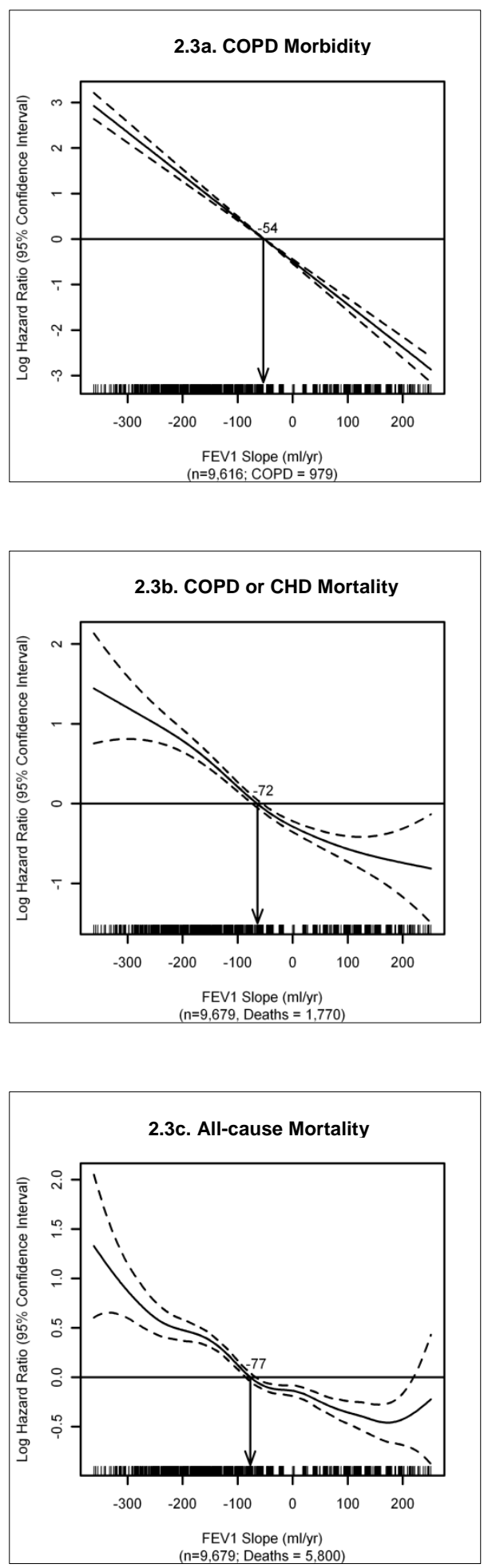

Figure 2.3 Cox proportional hazards model using a penalized spline for the FEV 1 slope, males and females combined. The spline crossed above zero for the log HR at $-54 \mathrm{ml} / \mathrm{yr}$ for (a) COPD morbidity, at $-72 \mathrm{ml} / \mathrm{yr}$ for (b) COPD or CHD mortality, and at $-77 \mathrm{ml} / \mathrm{yr}$ for (c) all-cause mortality. Models adjusted for baseline age, height-adjusted baseline lung function ( $\mathrm{FEV}_{1} /$ height $\left.^{2}\right)$, and height. Tick marks on the $\mathrm{x}$ axis represent the frequency of the various $\mathrm{FEV}_{1}$ slope values. 
Chapter 3 


\section{Chapter 3}

\subsection{Introduction}

Lung function is a significant predictor of increased COPD and CHD morbidity and mortality and all-cause mortality. ${ }^{32-34,37,38}$ Spirometry is recommended for secondary prevention through early detection of abnormal lung function. ${ }^{87}$ As COPD onset is typically during middle age, spirometry testing is recommended for individuals age 45 or older who are current or former smokers or with chronic environmental or occupational exposures. Spirometry testing is also recommended for individuals who have respiratory symptoms that may suggest COPD, including chronic cough, sputum production, wheezing, or shortness of breath on exertion. ${ }^{88,89}$

Occupational respiratory health monitoring for obstructive lung disease can include spirometry and/or administering a respiratory symptom questionnaire. ${ }^{3,4}$ Longitudinal spirometry is recommended for at-risk worker populations, many of whom have lung function above predicted values, for early detection of excessive declines due to exposures such as occupational hazards and tobacco smoking. ${ }^{51,90}$ Prevention based on longitudinal spirometry interpretation requires knowledge of morbidity and mortality risks associated with excessive lung function decline, respiratory symptoms, and asthma.

COPD prevention ${ }^{91}$ is crucial with its prevalence, costs, and disability projected to increase with aging populations, ${ }^{92-94}$ yet questions remain about the development and course of disease and associated morbidity and mortality. The Lung Health Study demonstrated that sustained smoking cessation leads to decreased rates of lung function decline. ${ }^{95}$ A more recent study of the natural history of chronic airflow obstruction in cohorts beginning at adolescence and young adulthood 
demonstrated benefits of early smoking cessation over later cessation on the rate of $\mathrm{FEV}_{1}$ decline and showed that susceptible smokers often had respiratory symptoms at a young age and/or a respiratory diagnosis during follow-up. ${ }^{96}$ Another prospective study showed that low lung function in young, non-asthmatic adults predicted airflow obstruction at middle age. ${ }^{97}$ In our previous study, decreased level of lung function and increased rate of decline were both associated with increased risks of hospital diagnosis of COPD, COPD or CHD mortality, and allcause mortality with $15-20$ years of follow-up, ${ }^{98}$ suggesting that early COPD prevention is beneficial.

The purpose of this study is to further investigate associations observed in our previous study between respiratory health outcomes (lung function, current respiratory symptoms, and current asthma) and subsequent COPD morbidity based on hospitalizations, COPD or CHD mortality, and all-cause mortality. ${ }^{98}$ We studied the above associations using extended morbidity and mortality follow-up. We also studied the effects of age when excessive lung function decline was identified on the above associations, and the effects of the pattern and persistency of the excessive lung function decline, respiratory symptoms, and asthma ascertained during 25 years of spirometry follow-up on subsequent morbidity and mortality.

\subsection{Methods}

\subsubsection{Study Population}

The Copenhagen City Heart Study is a prospective study of cardiovascular disease in males and females age 20 years and older with four examinations (1976-78, 1981-83, 1991-94, and 200103). The initial sample $(19,698)$ was supplemented with 500 subjects age $20-24$ years at examination two and 3,000 subjects age 20-49 years at examination three. Each examination 
included spirometry and a self-administered questionnaire. An electronic spirometer (N 403 Monaghan, United States) was used at examinations one and two and was replaced by a dry wedge spirometer (Vitalograph, United Kingdom) at examination three. Three spirometric measurements were collected at each examination with at least two within $5 \%$ of one another. The highest values of $\mathrm{FEV}_{1}$ and FVC were included in the data set. Current asthma, current respiratory symptoms of chronic bronchitis, and smoking status were ascertained by selfadministered questionnaire. ${ }^{72}$ Ascertainment of shortness of breath was initiated at examination two and of cough and wheeze by examination three.

Morbidity data were from the National Patient Register. Mortality data were from the Civil Registration System and causes of death from the National Register of Causes of Death. Health outcomes in the current study were 1) primary and secondary COPD hospital diagnoses (ICD-8 491-492, and ICD-10 J41-J44); 2) COPD or CHD mortality (ICD-8 410-414, and ICD-10 I20I25) as the underlying or contributing cause; and 3) all-cause mortality. (Denmark transitioned directly from the $8^{\text {th }}$ to the $10^{\text {th }}$ revision of ICD in 1993.) Follow-up was through $5 / 8 / 2009$ for COPD morbidity, 12/31/2006 for cause-specific mortality, and 5/17/2009 for all-cause mortality. Further information on the Copenhagen City Heart Study is available elsewhere. ${ }^{69,}, 99$

\subsubsection{Statistical Methods}

We used Cox proportional hazards models to investigate associations between lung function decline and morbidity and mortality through three objectives: 1 ) reassessing the overall risks and critical cut-points at which morbidity and mortality start to increase using extended follow-up data; 2) evaluating the effect of age on the above associations; and 3) evaluating the effect of 
temporal patterns in lung function decline and reported respiratory symptoms on subsequent morbidity and mortality. Figure 3.1 describes the subcohorts used for studying each objective.

\subsubsection{Overall Risks and Critical Cut-points}

We related lung function decline to morbidity and mortality using spirometry data from examinations three and four (1991-94 and 2001-03) with extended morbidity and mortality follow-up through 2009 to reassess the critical cut-points in lung function decline associated with morbidity and mortality risks. These spirometry data are more in accord with recent American Thoracic Society/European Respiratory Society standards (Figure 3.1, Objective 1). ${ }^{69}$ The midstudy change in model of spirometer prevented calculation of a regression slope using data from all four examinations. ${ }^{98}$

Cox models were used to estimate the risks of morbidity or mortality in relation to quartiles of height-adjusted baseline $\mathrm{FEV}_{1}\left(\mathrm{FEV}_{1} /\right.$ height $^{3}$, a power of height found appropriate for these data), ${ }^{53}$ quartiles of $\mathrm{FEV}_{1}$ slope (difference in $\mathrm{FEV}_{1}$ between the two examinations, divided by time between the examinations), respiratory symptoms (yes/no), and asthma (yes/no). The reference category was the $75^{\text {th }}$ percentile and above for lung function. Data for respiratory symptoms and asthma were from examination four. Models were stratified by gender, conducted for never smokers separately, and adjusted for baseline age and height. In addition, Cox models using penalized splines identified specific rates of decline where risks for morbidity and mortality began to increase for males and females combined ${ }^{68,100}$ These and the following models were adjusted for baseline age, baseline $\mathrm{FEV}_{1} /$ height $^{3}$, and height. 


\subsubsection{Effect of Age}

To investigate the effect of age on the association between the respiratory outcomes and subsequent morbidity and mortality risks, Cox models were stratified by the age at baseline

spirometry using an age threshold relevant to COPD development ( $\leq 45$ and $>45$ years) ${ }^{88,89}$ For never smokers, the age cut-point was 50 years. Quartiles of the $\mathrm{FEV}_{1}$ slope were calculated separately for males, females, and never-smoker males and females. To obtain a sufficient number of events by stratum, lung function measurements from examinations one and two (1976-78 and 1981-83) (Figure 3.1, Objective 2) were used to establish the respiratory outcomes. As in the previous study, sensitivity analyses assessed the adjustment to the FEV 1 measurements for $1981 .^{98}$

\subsubsection{Effect of Temporal Patterns in Lung Function Decline}

To determine the effect of temporal patterns in respiratory outcomes over the 1976-2003 period on subsequent morbidity and mortality, Cox models included subjects present at all examinations and their patterns of lung function decline and respiratory symptoms (Figure 3.1, Objective 3). Temporal patterns were established using $\mathrm{FEV}_{1}$ slopes in two periods: 1) examination one to two [1976-78 and 1981-83] and 2) examination three to four [1991-94 and 2001-03]. Slopes were dichotomized as either less than or equal to the median (excessive decline) or above the median (normal decline). Four temporal patterns of lung function decline were established: 1) normal decline over both periods [reference category]; 2) excessive decline over period one only; 3) excessive decline over period two only; and 4) excessive decline over both periods. Similar patterns were established for respiratory symptoms with symptoms reported at examination two corresponding to period one and symptoms reported at examination three or four corresponding to period two. Baseline symptoms were excluded because shortness of breath was not 
ascertained. Analyses were conducted with PROC PHREG $(p<0.05)$ in SAS, version 9.2 (SAS Institute Inc, USA), and R software, version 2.7.2, for the penalized splines.

\subsection{Results}

Characteristics of the three subcohorts used for the study objectives (Figure 3.1) are shown in Table 3.1. The table describes the number of subjects in each subcohort, duration of follow-up, lung function and $\mathrm{FEV}_{1}$ slope values, and the frequency of respiratory symptoms and asthma. Subjects lost to follow-up in objectives one (51.7\%), two (21.1\%), and three (78.0\%) had a higher average age, lower average $\mathrm{FEV}_{1} /$ height $^{3}$, and a higher percentage of self-reported current asthma, respiratory symptoms, and smoking at baseline; each of these characteristics was statistically different $(p<0.001)$ from those for the studied subjects. Loss to follow-up was mainly due to mortality.

\subsubsection{Overall Risks and Critical Cut-points in Lung Function Decline}

Quartiles of the level of lung function $\left(\mathrm{FEV}_{1} /\right.$ height $\left.^{3}\right)$ at examination three and the $\mathrm{FEV}_{1}$ slope from examination three to four were associated with increasing risks of COPD morbidity and allcause mortality (Table 3.2). For the slope and COPD morbidity, risks were significantly elevated at the fourth quartile, losses of -77 and $-57 \mathrm{ml} / \mathrm{yr}$ or more in males and females, respectively. For all-cause mortality, risks increased from the third quartile of the slope for males and females, declines of -54 and $-41 \mathrm{ml} / \mathrm{yr}$, respectively. For symptoms and asthma, females had higher associated risks for COPD morbidity than males. There were too few events to model COPD or CHD mortality because of short follow-up (four-year average) and morbidity in never smokers (six-year average). Based on penalized splines, the cut-points where morbidity and mortality risks began to increase approximated an overall decline of $-49 \mathrm{ml} / \mathrm{yr}$ (Figures 3.2a and 3.2b). 


\subsubsection{Age-related Risks Associated with Lung Function Decline, Symptoms, and Asthma}

Overall, there was an increasing trend in morbidity and mortality risks with increasing quartiles of the $\mathrm{FEV}_{1}$ slope for both age strata and gender groups, after adjustment for baseline $\mathrm{FEV}_{1}$ (Table 3.3). The risks of COPD morbidity and all-cause mortality were elevated significantly from the second quartile of the slope regardless of age in males and females, but these risks were often highest in the younger female group. The risk of COPD or CHD mortality was significantly elevated mainly for the highest quartile. Chronic bronchitis was a significant predictor of COPD morbidity in males and females across both ages, and in younger never smokers, while shortness of breath tended to be significant in males and older females for morbidity and mortality. For never smokers, the risk of COPD morbidity was significantly increased in older individuals at the highest quartile of the slope, while asthma and chronic bronchitis demonstrated the greatest risks in the younger never smokers. Although there was often an increasing trend in the HRs, they did not reach statistical significance.

\subsubsection{Risks Associated with Temporal Patterns of Lung Function Decline and Symptoms}

There was an increasing trend in COPD morbidity and all-cause mortality risks across the four temporal patterns of the slopes from the first two and last two examinations (Figures 3.3a and 3.3b), but there were too few events to model COPD or CHD mortality. For both COPD morbidity and all-cause mortality, the risks were higher and the trend was steeper for males. Patterns for chronic bronchitis and shortness of breath demonstrated lower risks for morbidity and mortality than the slope patterns. Overall, morbidity risks from respiratory symptoms were greater than for mortality. 
There was not a clear increasing trend in risk with shortness breath with patterns 2,3 , and 4 . COPD morbidity risks in males were (no events for pattern 2), 3.20 (1.86-5.52), and 3.72 (1.01$10.00)$ and in females these were $1.70(0.52-5.54), 4.23$ (2.65-6.75), 3.18 (1.43-7.04), respectively. All-cause mortality risks were $0.41(0.06-2.97), 1.83(1.27-2.63)$, and $1.22(0.52-$ 2.86) in males and $0.62(0.20-1.95), 1.48(1.05-2.09)$, and $1.72(0.91-3.26)$ in females, respectively. Asthma was excluded from these models because it did not contribute significantly. The only significant HRs for never smokers were for all-cause mortality and slope pattern 2 [2.28 (1.18-4.42)] and pattern 4 [2.06 (1.04-4.07)].

\subsection{Discussion}

This study is a continued investigation of the relationship of respiratory health status (lung function, respiratory symptoms, and asthma) with subsequent COPD morbidity and mortality. ${ }^{98}$ Extended outcome follow-up through 2009 permitted reassessment of critical cut-points where lung function decline became associated with increased risks of morbidity and mortality, using spirometry data from the last two examinations (1991-94 and 2001-03). We also investigated the effects of age at which lung function decline was ascertained on the association and of temporal patterns in lung function decline and respiratory symptoms on subsequent morbidity and mortality. Our study results could be useful in clinical practice and disease prevention, providing information about the predictive nature of lung function measurements, respiratory symptoms, and asthma for morbidity and mortality. This study also adds to current knowledge on respiratory status as a predictor of morbidity and mortality in younger and aging populations.

For prevention, it is important to establish the morbidity and mortality risks associated with the lung function level and rate of decline. Our current results (based on data from examinations 
three and four) are generally consistent with our previous findings (based on data from examinations one and two), ${ }^{98}$ showing high COPD morbidity and mortality risks associated with lower lung function levels and excessive decline (Table 3.2). Morbidity and mortality risks increased with declines starting at -77 and $-54 \mathrm{ml} / \mathrm{yr}$ in males and -57 and $-41 \mathrm{ml} / \mathrm{yr}$ in females, respectively (Table 3.2). A cut-point of $-49 \mathrm{ml} / \mathrm{yr}$ was associated with both increased morbidity and mortality risk, overall (Figures 3.2a and 3.2b). Outcome follow-up in the current study was too brief to investigate risks in never smokers, as was possible in the previous study where we found significant associations.

The current results are similar to those of prior studies of lung function decline and all-cause mortality. There are very few population-based morbidity studies, even though morbidity indicates the cost of disease, regardless of whether disease increases mortality. Parallels to earlier studies include significant associations between $\mathrm{FEV}_{1}$ decline and COPD hospitalizations and mortality, with some studies reporting higher associations for females than for males. ${ }^{27-29,33}$ As for cut-points of increased risk, our results for males demonstrated increased mortality risks similar to those in a study of underground coal miners where the risk increased at $-60 \mathrm{ml} / \mathrm{yr}$ and was statistically significant at $-90 \mathrm{ml} / \mathrm{yr}$ or more. ${ }^{68}$

Recent evidence indicates that individuals susceptible to COPD tend to have respiratory symptoms and lower lung function at younger ages, ${ }^{96}$ but spirometry testing is usually recommended for individuals 45 years of age or older. Our results for individuals 45 years of age or younger demonstrate excessive decline was a significant predictor of morbidity and mortality (Table 3.3), comparable to the risks for the older individuals. By gender and age, morbidity and mortality risks were higher in females. Among younger individuals, females had higher risks, 
even with lower smoking prevalence, a possible manifestation of greater susceptibility to the effects of smoking. ${ }^{76,77}$

The high COPD morbidity and mortality risks associated with excessive lung function decline found in younger individuals ( $\leq 45$ years) is relevant to screening practices as spirometry testing is currently recommended for individuals age 45 or older who are current or former smokers or with chronic environmental or occupational exposures or for individuals who have respiratory symptoms that may suggest disease. ${ }^{88,89}$ Susceptible smokers in the Framingham Offspring cohort tended to have respiratory symptoms at a young age and/or a respiratory diagnosis during follow-up. ${ }^{96}$ In our study, symptoms of chronic bronchitis were significant predictors of COPD morbidity in younger individuals. Given that lung function decline was a significant predictor of morbidity and mortality after adjusting for asthma and respiratory symptoms in our study, and that low lung function in young, non-asthmatic adults predicts airflow obstruction at middle age, ${ }^{97}$ screening for abnormal lung function decline at earlier ages may be useful, especially in at-risk occupational populations where spirometry-based medical monitoring is conducted. These results also have implications for the criteria used to identify disease such as the GOLD Stage criteria which generally tend to underdiagnose COPD in individuals younger than 50 years of age. $^{101}$

Although individuals susceptible to COPD development tended to have respiratory symptoms and lower lung function at a young age, ${ }^{96}$ it is well-known that intervention on risk factors such as smoking cessation helps to reduce the rate of lung function decline. Our results confirm that excessive decline in those 45 years of age or younger is associated with increased, and possibly preventable, morbidity and mortality. A question then arises about how risks associated with 
temporal patterns of lung function decline compare, such as early excessive decline followed by normal decline versus persistent excessive decline when compared with normal lung function decline.

Our results show increasing morbidity and mortality risks with increasing persistence of excessive decline and respiratory symptoms in males and females. Even never smokers had a two-fold mortality risk with persistent excessive decline. Detection of early declines, such as these in the first 4-7 years, and intervention could help prevent the risks observed in the temporal patterns that demonstrated even greater risk. The morbidity and mortality risks associated with the temporal patterns of chronic bronchitis and shortness of breath did not demonstrate a clear trend, but were often significant.

Among the study's limitations, the age-stratified analyses and those for temporal patterns in lung function decline and respiratory symptoms included adjusted $\mathrm{FEV}_{1}$ values for 1981 as in the previous study. ${ }^{98}$ From sensitivity analyses, the adjustment appears to have resulted in an underestimation of the HRs. Three clear cases were the fourth quartiles of the slope for COPD morbidity in males over age 45 and for COPD or CHD mortality in females age 45 years and younger, and slope pattern 4 (excessive decline over both periods) for COPD morbidity in males. Also, the morbidity and mortality follow-up for the temporal pattern analyses was relatively short (six-year average), risks related to the patterns of lung function decline would likely increase with longer follow-up and aging of the cohort.

Calculation of the slope using only two spirometry measurements could be susceptible to measurement error, but if not excessive, the quartile groupings could have reduced this bias. Misclassification was possible through underdiagnosis of COPD morbidity and underreporting of 
COPD mortality which would bias the results toward the null and weaken associations. ${ }^{84} \mathrm{We}$ combined COPD and CHD mortality to address this limitation, as these diseases are often linked. ${ }^{60,61}$ Generalizability of the COPD morbidity results is limited to individuals with more severe disease because cases were defined by hospital COPD diagnosis. Risks of morbidity and mortality could have been underestimated due to self-selection of healthier subjects into the Copenhagen City Heart Study and due to subjects lost to follow-up, increasingly overrepresenting healthier subjects over time.

In conclusion, this study showed that both the level of lung function and lung function decline were significant risk factors for excess morbidity and mortality, demonstrating the need for prevention on both. Intervention on excessive lung function loss could help prevent these outcomes and prevent lung function impairment. Associations between lung function decline and morbidity and mortality were also demonstrated at younger ages, providing evidence that serial lung function testing before middle age may identify a number of at-risk individuals who could benefit from medical intervention. The increasing morbidity and mortality risks associated with the temporal patterns of lung function decline suggest that early detection of lung function decline and appropriate intervention could result in substantial risk reduction. 


\begin{tabular}{|c|c|c|c|c|}
\hline $\begin{array}{l}\text { Examination } 1 \\
1976-78\end{array}$ & $\begin{array}{l}\text { Examination } 2 \\
1981-83\end{array}$ & $\begin{array}{l}\text { Examination } 3 \\
1991-94\end{array}$ & $\begin{array}{l}\text { Examination } 4 \\
2001-03\end{array}$ & $\begin{array}{l}\text { End of Follow-up } \\
2006 \quad 2009\end{array}$ \\
\hline \multirow[t]{2}{*}{13,830 Subjects } & 12,354 Subjects & 9,521 Subjects & 5,326 Subjects & \\
\hline & & $\begin{array}{l}\text { Objective } 1 \\
\text { subcohort: } \\
4,557 \text { Subjects }\end{array}$ & $\begin{array}{l}\text { Spirometry and ascertainment } \\
\text { of asthma, chronic bronchitis, } \\
\text { cough, shortness of breath, } \\
\text { and wheeze }\end{array}$ & $\begin{array}{l}-\longrightarrow \\
-\longrightarrow\end{array} 219$ COPD Morbidity \\
\hline $\begin{array}{l}\text { Objective } 2 \\
\text { subcohort: } \\
\text { 10,311 Subjects }\end{array}$ & $\begin{array}{l}\text { Spirometry and ascertainment } \\
\text { of asthma, chronic bronchitis, } \\
\text { and shortness of breath }\end{array}$ & & & $\begin{array}{l}-\rightarrow 1,347 \text { COPD Morbidity } \\
7 \text { COPD or CHD Mortality } \\
-\rightarrow 6,537 \text { All-cause Mortality }\end{array}$ \\
\hline $\begin{array}{l}\text { Objective } 3 \\
\text { subcohort: } \\
\text { 2,719 Subjects }\end{array}$ & $\begin{array}{l}\text { Spirometry and ascertainment } \\
\text { of chronic bronchitis and } \\
\text { shortness of breath }\end{array}$ & & & $\begin{array}{l}-\rightarrow 175 \text { COPD Morbidity } \\
-\rightarrow 510 \text { All-cause Mortality }\end{array}$ \\
\hline
\end{tabular}




\begin{tabular}{|c|c|c|c|c|c|c|}
\hline \multirow{2}{*}{\multicolumn{2}{|c|}{ Subcohort 1: Subjects present for examinations three and four* }} & \multirow[t]{2}{*}{ Males } & \multicolumn{2}{|c|}{ Females } & \multicolumn{2}{|c|}{ Never smokers } \\
\hline & & & & & & \\
\hline Subjects $(\mathrm{n}, \%) \dagger$ & 1,924 & 42.2 & 2,633 & 57.8 & 1,254 & 27.5 \\
\hline Baseline age (mean, SD) & 52.1 & 13.5 & 54.2 & 13.6 & 51.9 & 14.6 \\
\hline \multicolumn{7}{|l|}{ Years of follow-up (mean, SD) } \\
\hline COPD morbidity & 6.2 & 1.5 & 6.3 & 1.4 & 6.5 & 1.1 \\
\hline All-cause mortality & 6.3 & 1.4 & 6.4 & 1.2 & 6.5 & 1.1 \\
\hline Baseline $\mathrm{FEV}_{1} /$ height $^{3} \mathrm{x}$ mean height ${ }^{3}, 1$ (median, SD) & 3.17 & 0.63 & 2.87 & 0.63 & $3.46 ; 2.92 \ddagger$ & $0.58 ; 0.58 \ddagger$ \\
\hline Slope $\mathrm{FEV}_{1}, \mathrm{ml} / \mathrm{yr}$ (median, SD) & -54 & 35 & -41 & 27 & $-49 ;-38+$ & $34 ; 27 \ddagger$ \\
\hline Asthma $(\mathrm{n}, \%) \S$ & 100 & 5.2 & 201 & 7.6 & 63 & 5.0 \\
\hline Chronic bronchitis (n, \%)§ & 295 & 15.3 & 345 & 13.1 & 97 & 7.7 \\
\hline Never smokers (n, \%)§ & 416 & 22.0 & 838 & 32.1 & $416 ; 838 \ddagger$ & $33.2 ; 66.8 *$ \\
\hline Former smokers $(\mathrm{n}, \%) \S$ & 803 & 42.6 & 955 & 36.6 & & \\
\hline Current smokers (n, \%)§ & 668 & 35.4 & 816 & 31.3 & & \\
\hline \multicolumn{7}{|c|}{ Subcohort 2: Subjects present for examinations one and two* } \\
\hline Subjects with baseline age $\leq 45($ or $\leq 50)(\mathrm{n}, \% \dagger)$ & 1,262 & 12.2 & 1,445 & 14.0 & 751 & 7.3 \\
\hline Baseline age (mean, SD) & 37.5 & 6.1 & 37.6 & 5.9 & 39.3 & 7.5 \\
\hline Subjects with baseline age $>45($ or $>50)(\mathrm{n}, \% \dagger)$ & 3,271 & 31.7 & 4,333 & 42.0 & 1,184 & 11.5 \\
\hline Baseline age (mean, SD) & 57.8 & 7.5 & 57.4 & 7.2 & 60.6 & 6.5 \\
\hline Slope $\mathrm{FEV}_{1}, \mathrm{ml} / \mathrm{yr}$ (median, SD)ף & -59 & 91 & -55 & 69 & $-57 ;-39 \ddagger$ & $95 ; 71+$ \\
\hline \multicolumn{7}{|l|}{ Subcohort 3: Subjects present for all examinations* } \\
\hline
\end{tabular}

*Spirometry tests were 4 to 7 years apart from examination one to two and 7 to 12 years apart from examination three to four.

$\dagger$ A total of 10,311 subjects were present for examinations one and two, 4,557 for examinations three and four, and 2,719 for all examinations.

\$ales; females.

$\S$ Ascertained at examination four.

ๆOverall median and standard deviation values, not age-stratified.

$\mathrm{FEV}_{1}$, forced expiratory volume in one second; 1, liters; $\mathrm{ml} / \mathrm{yr}$, milliliters/year; $\mathrm{SD}$, standard deviation. 


\begin{tabular}{|c|c|c|c|c|}
\hline \multirow{3}{*}{ COPD Morbidity } & \multirow{2}{*}{\multicolumn{2}{|c|}{$\begin{array}{c}\text { Males } \\
(\mathrm{n}=1,878 ; \text { COPD }=91)\end{array}$}} & \multirow{2}{*}{\multicolumn{2}{|c|}{$\begin{array}{c}\text { Females } \\
(\mathrm{n}=2,554 ; \mathrm{COPD}=128)\end{array}$}} \\
\hline & & & & \\
\hline & HR & $95 \% \mathrm{CI}$ & HR & $95 \% \mathrm{CI}$ \\
\hline Q1 Baseline $\mathrm{FEV}_{1} /$ height $^{3}$ & 1.00 & & 1.00 & \\
\hline Q2 Baseline $\mathrm{FEV}_{1} /$ height $^{3}$ & 2.46 & $(0.47-12.81)$ & 5.61 & $(1.25-25.13)$ \\
\hline Q3 Baseline $\mathrm{FEV}_{1} /$ height $^{3}$ & 12.91 & $(2.93-56.82)$ & 11.42 & $(2.64-49.38)$ \\
\hline Q4 Baseline $\mathrm{FEV}_{1} /$ height $^{3}$ & 27.14 & $(6.12-120.31)$ & 23.31 & $(5.29-102.66)$ \\
\hline Q1 FEV 1 slope & 1.00 & & 1.00 & \\
\hline Q2 $\mathrm{FEV}_{1}$ slope & 1.87 & $(0.93-3.73)$ & 1.16 & $(0.65-2.10)$ \\
\hline Q3 FEV 1 slope & 1.28 & $(0.60-2.72)$ & 1.71 & $(0.99-2.97)$ \\
\hline Q4 $\mathrm{FEV}_{1}$ slope & 3.87 & $(1.99-7.55)$ & 2.56 & $(1.55-4.24)$ \\
\hline Asthma & 1.75 & $(0.92-3.32)$ & 2.48 & $(1.59-3.88)$ \\
\hline Chronic bronchitis & 1.11 & $(0.69-1.80)$ & 1.62 & $(1.08-2.44)$ \\
\hline Cough & 1.44 & $(0.87-2.38)$ & 1.80 & $(1.17-2.77)$ \\
\hline Shortness of breath & 1.60 & $(0.91-2.83)$ & 2.18 & $(1.39-3.40)$ \\
\hline Wheeze & 2.53 & $(1.55-4.13)$ & 2.03 & $(1.31-3.14)$ \\
\hline \multirow[t]{2}{*}{ All-cause Mortality } & \multicolumn{2}{|c|}{$(\mathrm{n}=1,924 ;$ Deaths $=292)$} & \multicolumn{2}{|c|}{$(n=2,633 ;$ Deaths $=313)$} \\
\hline & HR & $95 \% \mathrm{CI}$ & HR & $95 \% \mathrm{CI}$ \\
\hline Q1 Baseline $\mathrm{FEV}_{1} /$ height $^{3}$ & 1.00 & & 1.00 & \\
\hline Q2 Baseline $\mathrm{FEV}_{1} /$ height $^{3}$ & 2.13 & $(1.20-3.77)$ & 1.50 & $(0.77-2.91)$ \\
\hline Q3 Baseline $\mathrm{FEV}_{1} /$ height $^{3}$ & 1.82 & $(1.03-3.22)$ & 2.13 & $(1.12-4.06)$ \\
\hline Q4 Baseline $\mathrm{FEV}_{1} /$ height $^{3}$ & 3.04 & $(1.72-5.36)$ & 3.68 & $(1.92-7.07)$ \\
\hline Q1 FEV Flope $_{1}$ & 1.00 & & 1.00 & \\
\hline Q2 $\mathrm{FEV}_{1}$ slope & 1.00 & $(0.69-1.45)$ & 1.19 & $(0.85-1.67)$ \\
\hline Q3 FEV 1 slope & 1.63 & $(1.15-2.32)$ & 1.53 & $(1.09-2.15)$ \\
\hline Q4 $\mathrm{FEV}_{1}$ slope & 1.96 & $(1.38-2.77)$ & 1.91 & $(1.37-2.67)$ \\
\hline Asthma & 0.63 & $(0.38-1.06)$ & 1.27 & $(0.86-1.87)$ \\
\hline Chronic bronchitis & 0.92 & $(0.68-1.26)$ & 1.21 & $(0.89-1.65)$ \\
\hline Cough & 1.06 & $(0.75-1.50)$ & 1.17 & $(0.84-1.63)$ \\
\hline Shortness of breath & 1.60 & $(1.10-2.33)$ & 1.37 & $(0.97-1.95)$ \\
\hline Wheeze & 1.30 & $(0.96-1.77)$ & 0.94 & $(0.69-1.28)$ \\
\hline
\end{tabular}

*Adjusted for baseline age and height.

$\dagger$ Asthma and respiratory symptoms ascertained at examination four and represented as dichotomous variables in the models. 

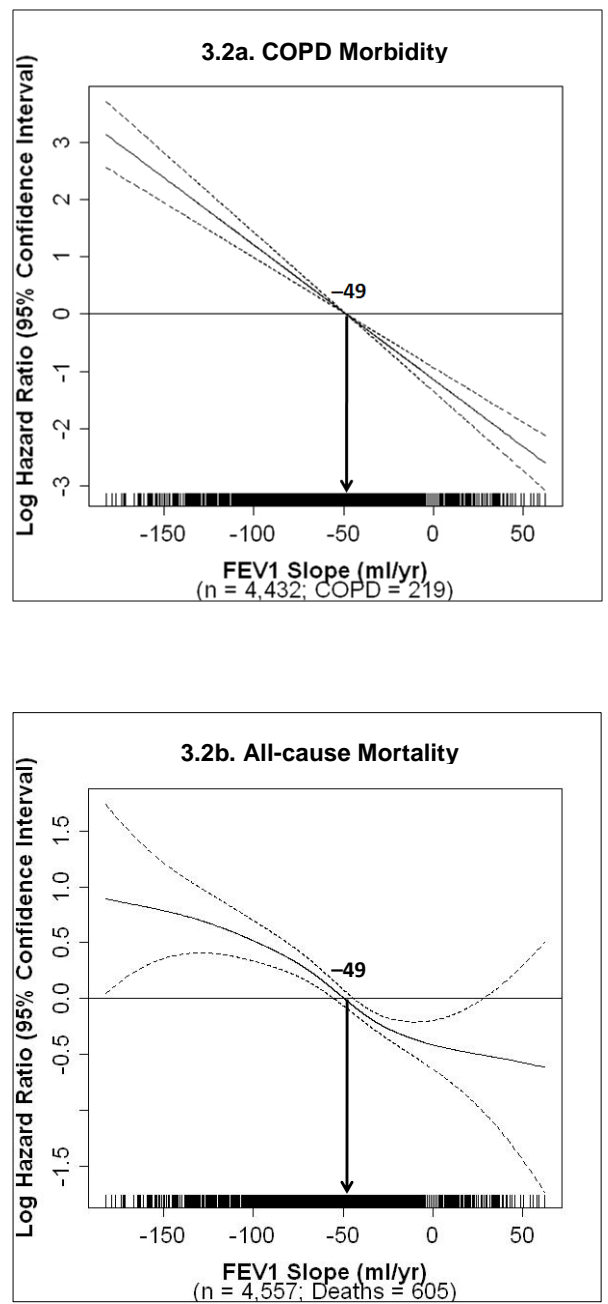

Figure 3.2 Cox proportional hazards model using a penalized spline for the FEV 1 slope, males and females combined. The spline crossed above zero for the log HR at $-49 \mathrm{ml} / \mathrm{yr}$ for (a) COPD morbidity and for (b) all-cause mortality. Models adjusted for baseline age, height-adjusted baseline lung function $\left(\mathrm{FEV}_{1} /\right.$ height $\left.^{3}\right)$, and height. Tick marks on the $\mathrm{x}$ axis represent the frequency of the various $\mathrm{FEV}_{1}$ slope values. 


\begin{tabular}{|c|c|c|c|c|c|c|c|c|c|c|c|c|}
\hline & \multicolumn{4}{|c|}{ Males } & \multicolumn{4}{|c|}{ Females } & \multicolumn{4}{|c|}{ Never Smokers } \\
\hline & \multicolumn{2}{|c|}{$\begin{array}{c}\text { Baseline Age } \\
\leq 45 \text { Years }\end{array}$} & \multicolumn{2}{|c|}{$\begin{array}{c}\text { Baseline Age } \\
>45 \text { Years }\end{array}$} & \multicolumn{2}{|c|}{$\begin{array}{c}\text { Baseline Age } \\
\leq 45 \text { Years }\end{array}$} & \multicolumn{2}{|c|}{$\begin{array}{c}\text { Baseline Age } \\
>45 \text { Years }\end{array}$} & \multicolumn{2}{|c|}{$\begin{array}{l}\text { Baseline Age } \\
\leq 50 \text { Years }\end{array}$} & \multicolumn{2}{|c|}{$\begin{array}{c}\text { Baseline Age } \\
>50 \text { Years }\end{array}$} \\
\hline COPD Morbidity & HR & $95 \% \mathrm{CI}$ & HR & $95 \% \mathrm{CI}$ & HR & $95 \% \mathrm{CI}$ & HR & $95 \% \mathrm{CI}$ & HR & $95 \% \mathrm{CI}$ & HR & $95 \% \mathrm{Cl}$ \\
\hline Q1 FEV 1 slope & 1.00 & & 1.00 & & 1.00 & & 1.00 & & 1.00 & & 1.00 & \\
\hline Q2 $\mathrm{FEV}_{1}$ slope & 1.30 & $(0.79-2.15)$ & 1.59 & $(1.17-2.15)$ & 2.18 & $(1.34-3.57)$ & 1.78 & $(1.36-2.33)$ & 0.82 & $(0.11-6.12)$ & 1.29 & $(0.57-2.89)$ \\
\hline Q4 $\mathrm{FEV}_{1}$ slope & 2.09 & $(1.18-3.70)$ & 4.03 & $(2.96-5.47)$ & 5.76 & $(3.41-9.74)$ & 4.57 & $(3.44-6.07)$ & 2.99 & $(0.48-18.72)$ & 2.86 & $(1.23-6.65)$ \\
\hline Asthma & 1.62 & $(0.56-4.65)$ & 1.54 & $(1.10-2.16)$ & 1.18 & $(0.57-2.43)$ & 0.96 & $(0.69-1.35)$ & 11.79 & $(2.22-62.63)$ & 2.60 & $(0.80-8.42)$ \\
\hline Chronic bronchitis & 1.90 & $(1.20-3.02)$ & 1.79 & $(1.43-2.24)$ & 3.09 & $(2.01-4.75)$ & 1.95 & $(1.59-2.40)$ & 6.06 & $(1.18-31.19)$ & 1.88 & $(0.82-4.31)$ \\
\hline Shortness of breath & 1.46 & $(0.75-2.85)$ & 1.73 & $(1.32-2.26)$ & 1.38 & $(0.85-2.26)$ & 2.24 & $(1.80-2.80)$ & 1.06 & $(0.11-9.87)$ & 1.26 & $(0.49-3.23)$ \\
\hline \multirow[t]{2}{*}{ COPD or CHD Mortality } & \multicolumn{2}{|c|}{$(\mathrm{n}=1,262 ;$ Deaths $=88)$} & \multicolumn{2}{|c|}{$(\mathrm{n}=3,271 ;$ Deaths=935) } & \multicolumn{2}{|c|}{$(\mathrm{n}=1,445 ;$ Deaths $=45)$} & \multicolumn{2}{|c|}{$(\mathrm{n}=4,333 ;$ Deaths $=789)$} & \multicolumn{2}{|c|}{$(\mathrm{n}=751 ;$ Deaths $=12)$} & \multicolumn{2}{|c|}{$(n=1,184 ;$ Deaths $=194)$} \\
\hline & HR & $95 \%$ CI & HR & $95 \%$ CI & HR & $95 \%$ CI & HR & $95 \%$ CI & HR & $95 \%$ CI & HR & $95 \% \mathrm{CI}$ \\
\hline Q1 $\mathrm{FEV}_{1}$ slope & 1.00 & & 1.00 & & 1.00 & & 1.00 & & 1.00 & & 1.00 & \\
\hline Q3 $\mathrm{FEV}_{1}$ slope & 1.80 & $(0.92-3.53)$ & 1.33 & $(1.09-1.63)$ & 1.59 & $(0.60-4.23)$ & 1.72 & $(1.36-2.17)$ & 2.91 & $(0.55-15.53)$ & 1.19 & $(0.77-1.83)$ \\
\hline Q4 FEV 1 slope & 2.03 & $(1.03-4.00)$ & 1.83 & $(1.50-2.25)$ & 4.29 & $(1.72-10.72)$ & 2.71 & $(2.14-3.44)$ & 0.58 & $(0.05-6.62)$ & 1.39 & $(0.88-2.19)$ \\
\hline Asthma & 2.09 & $(0.85-5.13)$ & 0.91 & $(0.67-1.23)$ & 1.08 & $(0.35-3.34)$ & 0.66 & $(0.47-0.92)$ & 1.79 & $(0.20-15.91)$ & 1.14 & $(0.46-2.84)$ \\
\hline Chronic bronchitis & 1.07 & $(0.60-1.89)$ & 1.33 & $(1.12-1.57)$ & 1.88 & $(0.86-4.12)$ & 1.50 & $(1.23-1.82)$ & 3.81 & $(0.72-20.10)$ & 1.15 & $(0.67-1.96)$ \\
\hline Shortness of breath & 3.58 & $(1.93-6.63)$ & 1.80 & $(1.48-2.19)$ & 1.78 & $(0.76-4.15)$ & 1.97 & $(1.61-2.40)$ & 2.32 & $(0.27-19.98)$ & 1.24 & $(0.72-2.14)$ \\
\hline \multirow[t]{2}{*}{ All-cause Mortality } & \multicolumn{2}{|c|}{$(\mathrm{n}=1,262 ;$ Deaths $=406)$} & \multicolumn{2}{|c|}{$(\mathrm{n}=3,271 ;$ Deaths $=2,750)$} & \multicolumn{2}{|c|}{$(\mathrm{n}=1,445 ;$ Deaths $=293)$} & \multicolumn{2}{|c|}{$(\mathrm{n}=4,333 ;$ Deaths $=3,088)$} & $(\mathrm{n}=75$ & ; Deaths $=110$ ) & $(\mathrm{n}=1,1$ & Deaths $=866$ ) \\
\hline & HR & $95 \% \mathrm{CI}$ & HR & $95 \% \mathrm{CI}$ & HR & $95 \% \mathrm{CI}$ & HR & $95 \%$ CI & HR & $95 \% \mathrm{CI}$ & HR & $95 \% \mathrm{Cl}$ \\
\hline Q1 FEV 1 slope & 1.00 & & 1.00 & & 1.00 & & 1.00 & & 1.00 & & 1.00 & \\
\hline Q2 $\mathrm{FEV}_{1}$ slope & 1.05 & $(0.80-1.38)$ & 1.16 & $(1.04-1.30)$ & 1.51 & $(1.08-2.10)$ & 1.23 & $(1.10-1.37)$ & 1.13 & $(0.66-1.95)$ & 1.12 & $(0.91-1.37)$ \\
\hline Q3 $\mathrm{FEV}_{1}$ slope & 1.43 & $(1.07-1.91)$ & 1.20 & $(1.07-1.34)$ & 1.53 & $(1.07-2.18)$ & 1.38 & $(1.23-1.55)$ & 1.33 & $(0.76-2.30)$ & 1.12 & $(0.91-1.38)$ \\
\hline Q4 FEV 1 slope & 1.77 & $(1.33-2.37)$ & 1.58 & $(1.41-1.78)$ & 2.15 & $(1.48-3.12)$ & 1.89 & $(1.68-2.12)$ & 1.38 & $(0.77-2.47)$ & 1.42 & $(1.14-1.76)$ \\
\hline Asthma & 1.03 & $(0.54-1.99)$ & 0.87 & $(0.70-1.07)$ & 0.98 & $(0.54-1.77)$ & 0.94 & $(0.76-1.15)$ & 1.41 & $(0.49-4.06)$ & 1.00 & $(0.62-1.61)$ \\
\hline Chronic bronchitis & 1.34 & $(1.03-1.76)$ & 1.24 & $(1.12-1.38)$ & 1.19 & $(0.80-1.76)$ & 1.27 & $(1.14-1.42)$ & 1.11 & $(0.40-3.06)$ & 1.12 & $(0.86-1.47)$ \\
\hline Shortness of breath & 1.78 & $(1.24-2.56)$ & 1.40 & $(1.23-1.60)$ & 1.37 & $(0.93-2.01)$ & 1.35 & $(1.20-1.52)$ & 1.25 & $(0.53-2.99)$ & 1.25 & $(0.96-1.63)$ \\
\hline
\end{tabular}

*Adjusted for baseline age, height-adjusted baseline lung function $\left(\mathrm{FEV}_{1} /\right.$ height $\left.^{3}\right)$, and height.

$\dagger$ Asthma and respiratory symptoms ascertained at examination two and represented as dichotomous variables in all models. 

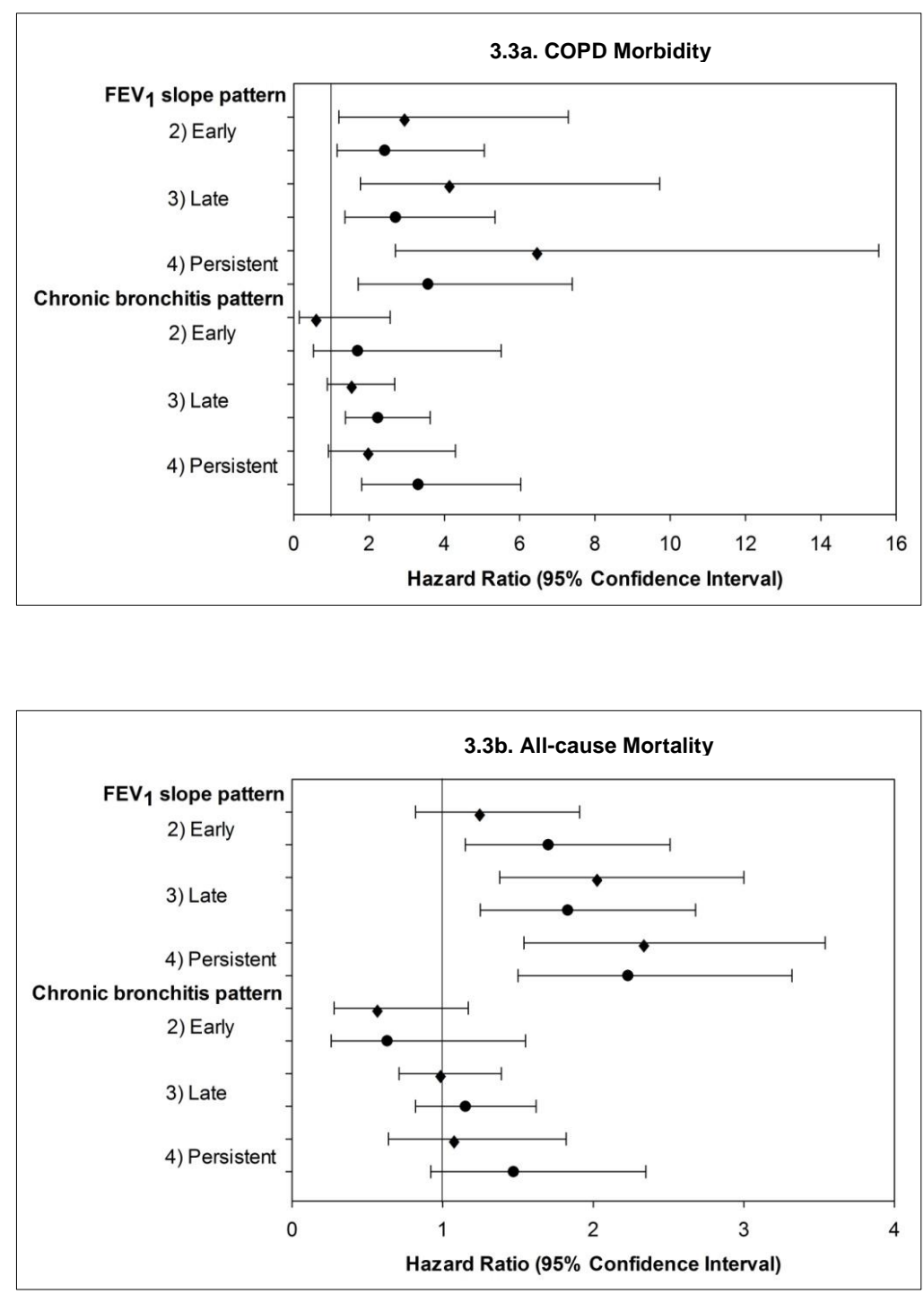

Figure 3.3 Cox proportional hazards models for patterns in lung function decline and respiratory symptoms (examination one to two and examination three to four) by gender $(=$ males and $\bullet=$ females) for (a) COPD morbidity and (b) all-cause mortality. Temporal patterns in the slope during the two follow-up periods: 1) normal decline over both periods [reference]; 2) excessive decline over period one only; 3 ) excessive decline over period two only; and 4) excessive decline over both periods. Temporal patterns in respiratory symptoms in the two follow-up periods: 1) absent in both periods [reference]; 2) present in period one only; 3) present in period two only; and 4) present in both periods. Models adjusted for baseline age, height-adjusted baseline lung function $\left(\mathrm{FEV}_{1} /\right.$ height $\left.^{3}\right)$, and height. 
Chapter 4 


\section{Chapter 4}

\subsection{Introduction}

Lung function is a significant predictor of COPD and CHD morbidity and mortality and all-cause mortality. ${ }^{24-39,55-57,102}$ Generally, the absolute level of forced expiratory volume in one second $\left(\mathrm{FEV}_{1}\right)$ is indicative of severity of airflow obstruction and COPD, while the rate of FEV $\mathrm{C}_{1}$ decline is a marker of disease progression. COPD is a costly, highly-prevalent disease worldwide $7,11,58$, 59, 103 and multifactorial in nature. COPD is generally caused by inflammatory processes in the lungs initiated by hazardous exposures that may lead to chronic bronchitis, small airways disease, or emphysema. 1,104

Both emphysema and small airways disease are associated with gradual loss of tissue elasticity combined with collagen deposition, leading to airflow limitation. ${ }^{105}$ Changes usually occur gradually, generally before abnormality can be detected with spirometry or radiology. Early markers suitable for detection of the disease processes are not yet available for widespread application, although research in this area is progressing. ${ }^{106,107}$ Furthermore, the strong association between COPD and cardiovascular disease suggests that impaired lung function caused by inflammatory changes in the lungs may lead to increased systemic inflammation contributing to non-pulmonary disease. ${ }^{108}$

COPD is complex, with several early indicators of risk, including airflow limitation, respiratory symptoms, and asthma, and known risk factors such as smoking, and environmental and occupational exposures. Spirometry testing is essential for diagnosis and monitoring of COPD progression. ${ }^{1}$ Monitoring lung function over time in at-risk individuals can help identify 
underlying progressive disease before lung function becomes abnormal. Combining the results of cross-sectional and longitudinal spirometry could be informative for early identification of disease and allow for intervention on patterns that may be associated with increased morbidity or mortality risks. This approach would be particularly important for individuals who have lung function levels above predicted values. ${ }^{51,90,109,110}$

The purpose of this study was to evaluate the morbidity and mortality risks associated with the combined effect of the level of lung function and the rate of lung function decline. Lung function categories, from baseline forced expiratory volume in one second $\left(\mathrm{FEV}_{1 \mathrm{~b}}\right)$ compared to predicted values, and quartiles of $\mathrm{FEV}_{1}$ slope, were used to identify potential high-risk groups for prevention. Furthermore, the relationship between the lung function categories and the prevalence rates of respiratory symptoms was investigated.

\subsection{Methods}

\subsubsection{Study Population}

The Copenhagen City Heart Study is a 28-year cardiovascular disease study with four examinations of males and females age 20 years and older. The primary sample $(19,698)$ was drawn randomly from the Copenhagen Population Register in five-year age groups. Clinical examinations (with spirometry) and a self-administered questionnaire were conducted. An electronic spirometer (N 403 Monaghan, United States) was used for examinations one and two (1976-78 and 1981-83). At each examination, three spirometric measurements were obtained with at least two within $5 \%$ of one another. The highest values of $\mathrm{FEV}_{1}$ and $\mathrm{FVC}$ were retained in the data set. Age and gender data were from the Copenhagen Population Register at study enrollment and height was measured during the clinical examination. Smoking status and current 
respiratory symptoms of chronic bronchitis were ascertained by questionnaire at each examination, and shortness of breath was ascertained beginning at examination two. ${ }^{69,72}$

Morbidity data were from the National Patient Register. Mortality data were from the Civil Registration System and cause-specific mortality data were from the National Register of Causes of Death. In the current study, health outcomes are 1) primary and secondary COPD hospital diagnoses (ICD-8 491-492, and ICD-10 J41-J44); 2) COPD or CHD mortality (ICD-8 410-414, and ICD-10 I20-I25) as the underlying or contributing cause; and 3) all-cause mortality.

(Denmark transitioned directly from the $8^{\text {th }}$ to the $10^{\text {th }}$ revision of ICD in 1993.) Follow-up lasted through 5/8/2009 for COPD morbidity, 12/31/2006 for cause-specific mortality, and 5/17/2009 for all-cause mortality. Additional Copenhagen City Heart Study information is available elsewhere. $^{69,99}$

\subsubsection{Statistical Methods}

We used Cox proportional hazards models to estimate morbidity and mortality risks associated with the combined effect of the $\mathrm{FEV}_{1 \mathrm{~b}}$ and subsequent $\mathrm{FEV}_{1}$ slope (difference in $\mathrm{FEV}_{1}$ between the first two examinations, divided by time between the examinations) with an average follow-up of five years (Figure 4.1). Combinations of $\mathrm{FEV}_{1 \mathrm{~b}}$ in comparison to predicted values ${ }^{111}$ and quartiles of $\mathrm{FEV}_{1}$ slope formed nine categories in a single variable representing a continuum of respiratory health status (Figure 4.2). The reference category included individuals with the most robust lung function. Analyses were based on $\mathrm{FEV}_{1}$ measurements because these are the most effective for evaluation of lung function decline. ${ }^{71}$ Measurements from examinations one and two (1976-78 and 1981-83) were used to achieve a sufficient number of health outcomes by category. 
Hazard ratios (HR) were estimated and corresponding 95\% confidence intervals (CI) were constructed overall, by gender, for never smokers, and by baseline age ( $\leq 45$ and $>45)$, an age breakpoint related to COPD development. ${ }^{88,89}$ Time to event (or censor) was from examination two until COPD hospital diagnosis, death, or end of follow-up for COPD morbidity and until death or end of follow-up for mortality. All models used gender-specific quartile values for the $\mathrm{FEV}_{1}$ slope, and were adjusted for baseline age and height. Other possible confounders, such as smoking, were not included because we were interested purely in the relationships of the lung function categories with morbidity and mortality for prevention purposes.

We also calculated Spearman's rho rank correlation coefficients between the nine categories and the overall prevalence rates of current respiratory symptoms of chronic bronchitis and shortness of breath at examination two. Population attributable risks (PAR) were calculated with Levin's formula ${ }^{112}$ for category 9 and jointly for categories 8 and 9 as an example of the potential contribution of an accelerated slope in those with a 'normal' $\mathrm{FEV}_{1 \mathrm{~b}}$. As in our previous study, sensitivity analyses assessed the effect of an adjustment to $\mathrm{FEV}_{1}$ measurements for 1981 that were initially higher on average than those at examinations one (1976-78) and two (1982 and 1983). ${ }^{98}$

All Copenhagen City Heart Study subjects gave informed consent to participate. The Study was performed in accordance with the $2^{\text {nd }}$ Helsinki Declaration and approved by the regional ethics committee. Current analyses were approved by the National Institute for Occupational Safety and Health Human Subjects Review Board and the West Virginia University Institutional Review Board. All analyses were conducted with PROC PHREG $(p<0.05)$ in SAS, version 9.2 (SAS Institute Inc, USA). 


\subsection{Results}

The characteristics of the 10,457 individuals with spirometry testing at examinations one and two are summarized by subcohort (males, females, and never smokers) in Table 4.1. The table describes the sample sizes, duration of morbidity and mortality follow-up (16-21 years), genderspecific values for $\mathrm{FEV}_{1 \mathrm{~b}}$ and the quartiles of $\mathrm{FEV}_{1}$ slope, and smoking status. Of note are the high prevalence rates of current and former smoking, making this largely an at-risk sample. Individuals lost to follow-up by examination two (22.9\%) had a higher average age, lower average height-adjusted $\mathrm{FEV}_{1}$, and higher prevalence rates of self-reported current respiratory symptoms of chronic bronchitis, current asthma, and smoking at baseline; each of these characteristics was significantly different $(p<0.001)$ as compared to study subjects.

\subsubsection{Overall Morbidity and Mortality Risks}

Significant overall trends in the risks of morbidity and mortality outcomes across the nine lung function categories are demonstrated in Figures 4.3a-c. Risks were higher for COPD morbidity as compared to the mortality outcomes. Statistically significant increases in the morbidity and mortality risks began at category $4\left(\mathrm{FEV}_{1 \mathrm{~b}}\right.$ at or above predicted and the fourth quartile of the slope). By category 8 (FEV $1 \mathrm{~b}$ below predicted but at or above the LLN and the fourth quartile of the slope), HRs reached 7.29 (4.24-12.52) for COPD morbidity, 4.07 (2.70-6.13) for COPD or CHD mortality, and 2.13 (1.80-2.53) for all-cause mortality. The PARs for category 8 and above were considerably increased from those for category 9, even though the HRs were similar (Table 4.2). The overall prevalence rates of symptoms of chronic bronchitis and shortness of breath also generally increased with worsening lung function categories (Figure 4.4). The Spearman's rho rank correlations were both $-0.98(p<0.0001)$. 


\subsubsection{Morbidity and Mortality Risks by Gender, for Never Smokers, and by Baseline Age at Spirometry Testing}

Morbidity and mortality risks by gender and for never smokers are presented in Table 4.3. In general, an increasing trend in the HRs across the lung function categories was also observed for these subcohorts. By gender, the risk of COPD morbidity was higher overall for females, where the risk was statistically significant starting at category $4\left(\mathrm{FEV}_{1 \mathrm{~b}}\right.$ at or above predicted and the fourth quartile of the slope). Among never smokers, there was a clear trend for increasing COPD morbidity risk with worsening lung function category, however the risk was only significant with category $9\left(\mathrm{FEV}_{1 \mathrm{~b}}\right.$ below the LLN). When stratified by gender, females demonstrated higher COPD or CHD mortality risks than males; this pattern was not as clearly seen for all-cause mortality.

Morbidity and mortality risks stratified by baseline age $(\leq 45$ and $>45)$ at spirometry testing are shown in Table 4.4. The general increasing trend in the HRs across the lung function categories was found in both the younger and older age groups. Among each subcohort (males, females, never smokers, and ages $\leq 45$ and $>45$ ), cause-specific and all-cause mortality risks were significantly increased for those with 'normal' lung function ( $\mathrm{FEV}_{1 \mathrm{~b}}$ at or above the LLN), but with accelerated $\mathrm{FEV}_{1}$ decline (fourth quartile of the slope).

Throughout these models, our method of stratification by combinations of lung function level and rate of decline created the potential for a relatively small sample size in the reference category that could influence the results. For example, this may partially explain the genderspecific differences in the morbidity and mortality risks. To explore this issue, we created a new reference category by combining categories 1 and 2 . The addition of category 2 narrowed the 
gender gap in risks slightly and reduced the HRs. Cumulative incidence of morbidity and mortality within each lung function category was calculated for males, females, and never smokers to provide more information related to this issue (Figure 4.5). To test the possibility that the elevated risks in those with 'normal' $\mathrm{FEV}_{1 \mathrm{~b}}$ was due to airflow obstruction, we repeated the overall analyses excluding those with $\mathrm{FEV}_{1 \mathrm{~b}}$ above the $\mathrm{LLN}$, but with a ratio of $\mathrm{FEV}_{1 \mathrm{~b}} /$ baseline FVC $\left(\mathrm{FVC}_{\mathrm{b}}\right)$ below the LLN. The results changed very little.

\subsection{Discussion}

This study shows that excessive rates of $\mathrm{FEV}_{1}$ decline are associated with increased morbidity and mortality risks, even with a 'normal' baseline $\mathrm{FEV}_{1}$ (Figure 4.3). Increasing trends in risk were consistently identified with baseline $\mathrm{FEV}_{1}$ at or above the LLN and increasing quartiles of decline in males, females, never smokers, and with a baseline age of 45 or under or above 45 (Tables 4.3-4.4). To estimate the contribution of an accelerated decline in those with a 'normal' $\mathrm{FEV}_{1}$, we calculated and compared the PARs ${ }^{112}$ for $\mathrm{FEV}_{1}$ below the LLN, and then jointly with a 'normal' $\mathrm{FEV}_{1}$ and the steepest declines (categories 8 and 9). The additional contribution was $12.0 \%$ for COPD morbidity (49.6-37.6\%, Table 4.2$), 9.6 \%$ for COPD or CHD mortality, and $7.1 \%$ for all-cause mortality; indicating that the contribution of accelerated $\mathrm{FEV}_{1}$ decline in those with 'normal' $\mathrm{FEV}_{1}$ has public health significance.

Although previous studies show associations between morbidity and mortality and $\mathrm{FEV}_{1}$ and the rate of decline, ${ }^{24-39,55-57,102}$ evaluating their combined effect is a new approach and adds to current knowledge regarding prevention. Combining baseline $\mathrm{FEV}_{1}$ and the rate of decline, we could investigate the importance of accelerated decline despite 'normal' baseline FEV (Figure $^{2}$ 4.2). Similar to prior studies, we also identified higher morbidity and mortality risks among 
females (Table 4.3), ${ }^{29,33}$ which may result from increased susceptibility to the effects of smoking, ${ }^{77}$ and increased COPD morbidity risk with lower lung function at younger ages ${ }^{96}$ (Table 4.4).

Most chronic conditions affecting lung function progress and accelerate with age. With COPD, chronic inflammatory processes in the lungs lead to elastic tissue loss and small airways remodeling, ${ }^{105}$ progressive conditions starting years before abnormalities are detectible with spirometry or radiology. It has been suggested that inflammation in the lungs may contribute to systemic inflammation, promoting non-pulmonary disease, such as ischemic heart disease. ${ }^{108}$ Currently there are no useful early markers of these disease processes. Many individuals with abnormal spirometry results may follow a normal trajectory with age, while others with 'normal' results may display excessive decline likely reflecting progressive disease. Early COPD detection may be improved through longitudinal spirometry testing in at-risk populations (smokers, occupationally-exposed groups, individuals with respiratory symptoms [Figure 4.4]). ${ }^{21,98}$ Well-established risk factors for excessive decline are similar to those for COPD (smoking, asthma, childhood respiratory diseases, occupational and environmental exposures). ${ }^{1}$ ${ }^{33,34,96}$ Longitudinal spirometry may identify individuals with a high level of lung function but excessive decline, whose disease could progress undetected over many years and eventually lead to increased morbidity and mortality risks (Tables 4.3-4.4). ${ }^{51,109}$

Barriers to the implementation of longitudinal spirometry include cost, data standardization and quality, and effective interpretation of results. ${ }^{109}$ However, longitudinal spirometry could result in both future cost-savings in at-risk or previously-diagnosed populations and improved quality 
of life through interventions that preserve lung function. Additionally, computerized spirometry data can facilitate data interpretation and higher data quality. ${ }^{113}$

Despite this study's strengths, such as a large, age-stratified population and long outcome follow-up, there were limitations including the adjustment of $\mathrm{FEV}_{1}$ values for $1981 .^{98}$ Sensitivity analyses indicated under- and overestimation of COPD morbidity and COPD or CHD mortality risks but was not possible for COPD or CHD mortality risk in never smokers because there were no events in the reference category of the comparison model. The overestimations of risk were for COPD morbidity risk in never smokers (categories 8 and 9) and for COPD or CHD mortality risk in individuals with a baseline age of 45 or younger (categories 6, 8, and 9) (Tables 4.3 and 4.4). Underestimation of morbidity and mortality risks could also have occurred through selfselection of healthier subjects into the Copenhagen City Heart Study and subjects lost to followup.

As COPD morbidity is generally underdiagnosed and mortality underreported, misclassification could have biased the results toward the null and decreased associations. ${ }^{84}$ Thus, the COPD or CHD mortality outcome was used. ${ }^{60,61,102}$ COPD morbidity defined as a hospital diagnosis limits the generalizability of the results to individuals with more severe disease. The $\mathrm{FEV}_{1}$ slope calculated from only two measurements is vulnerable to measurement error, although use of quartiles may have lessened this effect. Also, individuals with abnormal baseline lung function were included in the models. Overall Cox models were conducted post hoc excluding: 1) individuals with a ratio of $\mathrm{FEV}_{1 \mathrm{~b}} / \mathrm{FVC}_{\mathrm{b}}$ below the $\mathrm{LLN}$ and then 2) individuals with $\mathrm{FEV}_{1 \mathrm{~b}}$ and $\mathrm{FEV}_{\mathrm{lb}} / \mathrm{FVC}_{\mathrm{b}}$ below the LLN. These results differed mainly by lower HRs in category 9 . 
In conclusion, this study demonstrates the usefulness of a combined cross-sectional and longitudinal evaluation of $\mathrm{FEV}_{1}$ in an aging, community-based cohort. Combinations of decreasing baseline $\mathrm{FEV}_{1}$ and increasing $\mathrm{FEV}_{1}$ slope showed steadily increasing trends in morbidity and mortality risks. The combined approach may provide more useful information for early identification of disease, even in individuals with a 'normal' $\mathrm{FEV}_{1}$. These study results may be useful to health care providers who evaluate patients at risk for lung function decline. 


$\begin{array}{ll}\begin{array}{l}\text { Examination } 1 \\ 1976-78\end{array} & \begin{array}{l}\text { Examination } 2 \\ 1981-83\end{array} \\ 13,830 \text { Subjects } & 12,354 \text { Subjects } \\ 2006 & \text { End of Follow-up } \\ 2009\end{array}$

Figure 4.1 Sample size and morbidity and mortality follow-up. Above the timeline are the numbers of subjects who participated in spirometry testing by Copenhagen City Heart Study examination. Below the timeline is the number of subjects who participated in spirometry testing at examinations one and two and the numbers of health outcomes that occurred during the morbidity and mortality follow-up periods. Note that the follow-up periods are represented as dashed lines and that followup for COPD or CHD mortality ended in 2006. 


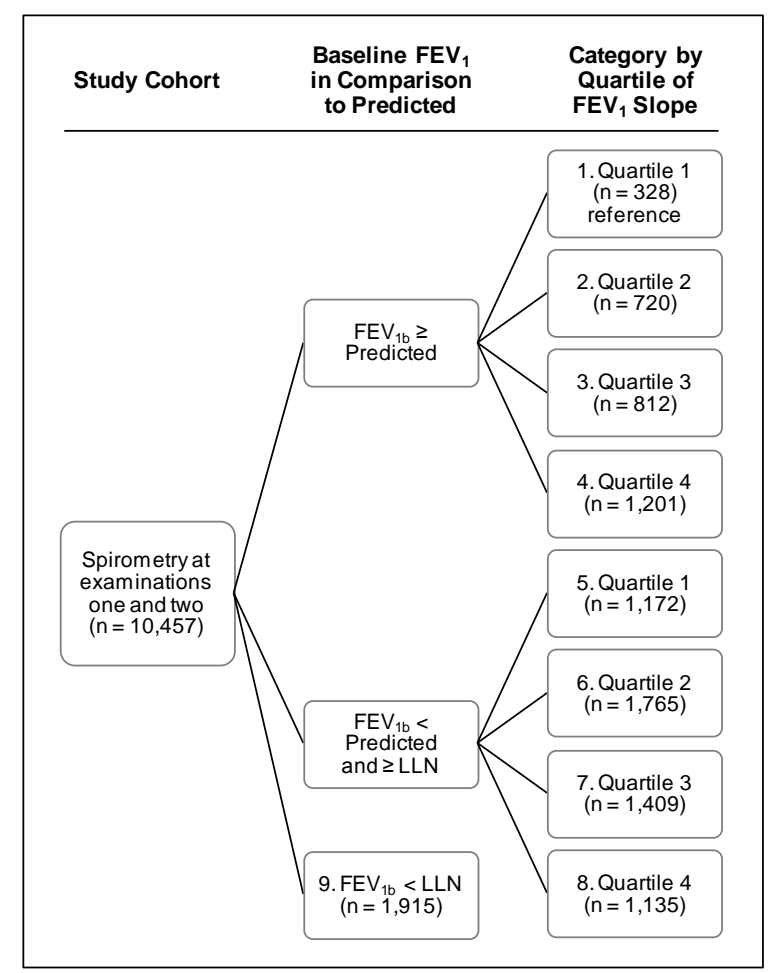

Figure 4.2 Nine lung function categories based on baseline $\mathrm{FEV}_{1}$ values in comparison to predicted values and quartiles of $F E V_{1}$ slope. European reference equations were used to calculate the predicted $F E V_{1}$ values. ${ }^{111} \mathrm{FEV}_{1 \mathrm{~b}}$, baseline forced expiratory volume in one second; LLN, lower limit of normal. 


\begin{tabular}{|c|c|c|c|c|c|c|}
\hline \multirow{2}{*}{ Subjects present for examinations one and twe } & \multicolumn{2}{|c|}{ Males } & \multicolumn{2}{|c|}{ Females } & \multicolumn{2}{|c|}{ Never smokers } \\
\hline & & & & & & \\
\hline Subjects $(\mathrm{n}, \%) \dagger$ & 4,598 & 44.0 & 5,859 & 56.0 & 1,949 & 18.6 \\
\hline Baseline age (mean, SD) & 52.2 & 11.6 & 52.4 & 11.0 & 52.3 & 12.4 \\
\hline Subjects with baseline age $\leq 45$ (mean, SD) & 37.5 & 6.0 & 37.6 & 5.9 & 36.4 & 6.4 \\
\hline Subjects with baseline age $>45$ (mean, SD) & 57.8 & 7.5 & 57.4 & 7.2 & 58.8 & 7.5 \\
\hline COPD morbidity & 17.0 & 8.8 & 19.6 & 7.9 & 21.1 & 7.7 \\
\hline COPD or CHD mortality & 16.7 & 7.9 & 19.2 & 6.9 & 20.1 & 6.7 \\
\hline All-cause mortality & 17.5 & 8.7 & 20.2 & 7.8 & 21.3 & 7.6 \\
\hline $\mathrm{FEV}_{1 \mathrm{~b}}, 1$ (mean, SD) & 3.09 & 0.83 & 2.25 & 0.55 & $3.48 ; 2.31 \ddagger$ & $0.85 ; 0.55 t$ \\
\hline $\mathrm{FEV}_{\mathrm{lb}}, \%$ predicted (mean, SD) & 88.6 & 18.2 & 91.9 & 17.8 & $95.0 ; 96.6 \$$ & $15.9 ; 18.0 \%$ \\
\hline Q3 Slope FEV $1(n, \%)$ & 317 & 26.7 & 495 & 26.4 & 212 & 26.8 \\
\hline Q4 Slope FEV $1(n, \%)$ & 449 & 37.8 & 752 & 40.2 & 322 & 40.7 \\
\hline $\mathrm{FEV}_{1 \mathrm{~b}}<$ predicted and $\geq \operatorname{LLN}(\mathrm{n}, \%)$ & 2,420 & 52.6 & 3,061 & 52.2 & 962 & 49.4 \\
\hline Q1 Slope FEV 1 (n, \%) & 528 & 21.8 & 644 & 21.0 & 241 & 25.1 \\
\hline Q2 Slope FEV $1(n, \%)$ & 735 & 30.4 & 1,030 & 33.6 & 320 & 33.3 \\
\hline Q3 Slope FEV 1 (n, \%) & 606 & 25.0 & 803 & 26.2 & 248 & 25.8 \\
\hline Q4 Slope FEV 1 (n, \%) & 551 & 22.8 & 584 & 19.1 & 153 & 15.9 \\
\hline $\mathrm{FEV}_{1 \mathrm{~b}}<\mathrm{LLN}(\mathrm{n}, \%)$ & 989 & 21.5 & 926 & 15.8 & 196 & 10.1 \\
\hline Slope $\mathrm{FEV}_{1}, \mathrm{ml} / \mathrm{yr}$ (mean, SD) & -61 & 91 & -44 & 69 & $-48 ;-40 \ddagger$ & $95 ; 71+$ \\
\hline $25^{\text {th }}$ percentile & -117 & & -94 & & $-113 ;-81 \ddagger$ & \\
\hline
\end{tabular}

*Spirometry tests were 4 to 7 years apart from examination one to two with a mean follow-up of 5 years.

$\dagger$ A total of 10,457 subjects were present for examinations one and two.

\$ales; females.

$\S$ Ascertained at examination two.

$\mathrm{FEV}_{1 \mathrm{~b}}$, baseline forced expiratory volume in one second; 1, liters; LLN, lower limit of normal; ml/yr, milliliters/year; SD, standard deviation. 

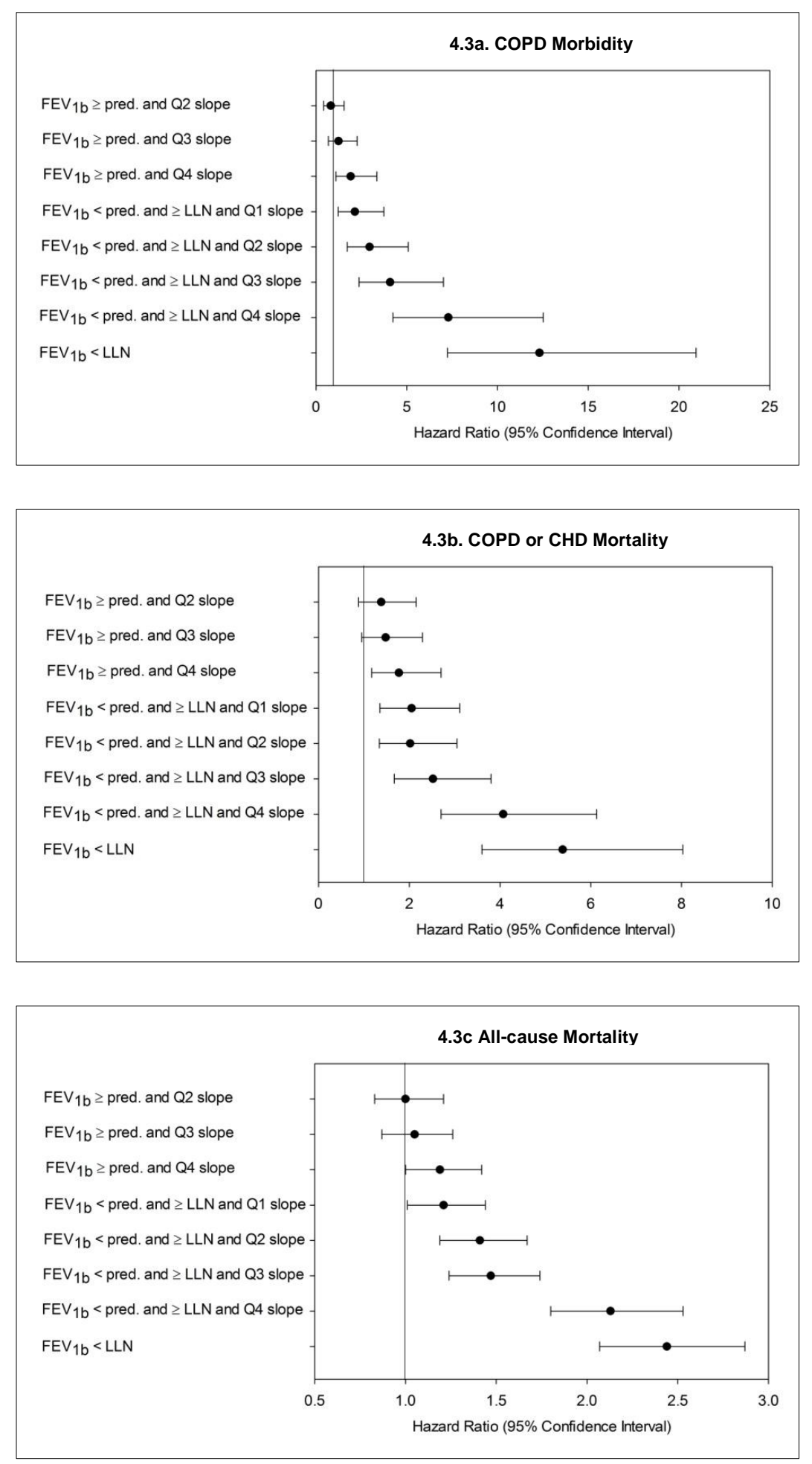

Figure 4.3. Cox proportional hazards model results for overall risks of (a) COPD morbidity, (b) COPD or CHD mortality, and c) allcause mortality by lung function category. Models adjusted for baseline age and height. FEV $\mathrm{fb}_{\mathrm{b}}$, baseline forced expiratory volume in one second; LLN, lower limit of normal; pred., predicted value. 


\begin{tabular}{|c|c|c|c|}
\hline & HR & $95 \% \mathrm{CI}$ & PAR (\%) \\
\hline COPD Morbidity & $(\mathrm{n}=10,38$ & $\mathrm{OPD}=1,405)$ & \\
\hline Categories 1-8 & 1.00 & & \\
\hline Category 9 & 4.35 & $(3.90-4.85)$ & 37.6 \\
\hline Categories 1-7 & 1.00 & & \\
\hline Categories 8 and 9 & 4.42 & $(3.97-4.91)$ & 49.6 \\
\hline COPD or CHD Mortality & $(\mathrm{n}=10,45$ & eaths $=1,915)$ & \\
\hline Categories 1-8 & 1.00 & & \\
\hline Category 9 & 2.48 & $(2.24-2.74)$ & 21.3 \\
\hline Categories 1-7 & 1.00 & & \\
\hline Categories 8 and 9 & 2.53 & $(2.31-2.78)$ & 30.9 \\
\hline All-cause Mortality & $(\mathrm{n}=10,45$ & eaths $=6,666)$ & \\
\hline Categories 1-8 & 1.00 & & \\
\hline Category 9 & 1.80 & $(1.70-1.91)$ & 12.8 \\
\hline Categories 1-7 & 1.00 & & \\
\hline Categories 8 and 9 & 1.85 & $(1.75-1.95)$ & 19.9 \\
\hline
\end{tabular}

*Models adjusted for baseline age and height. 


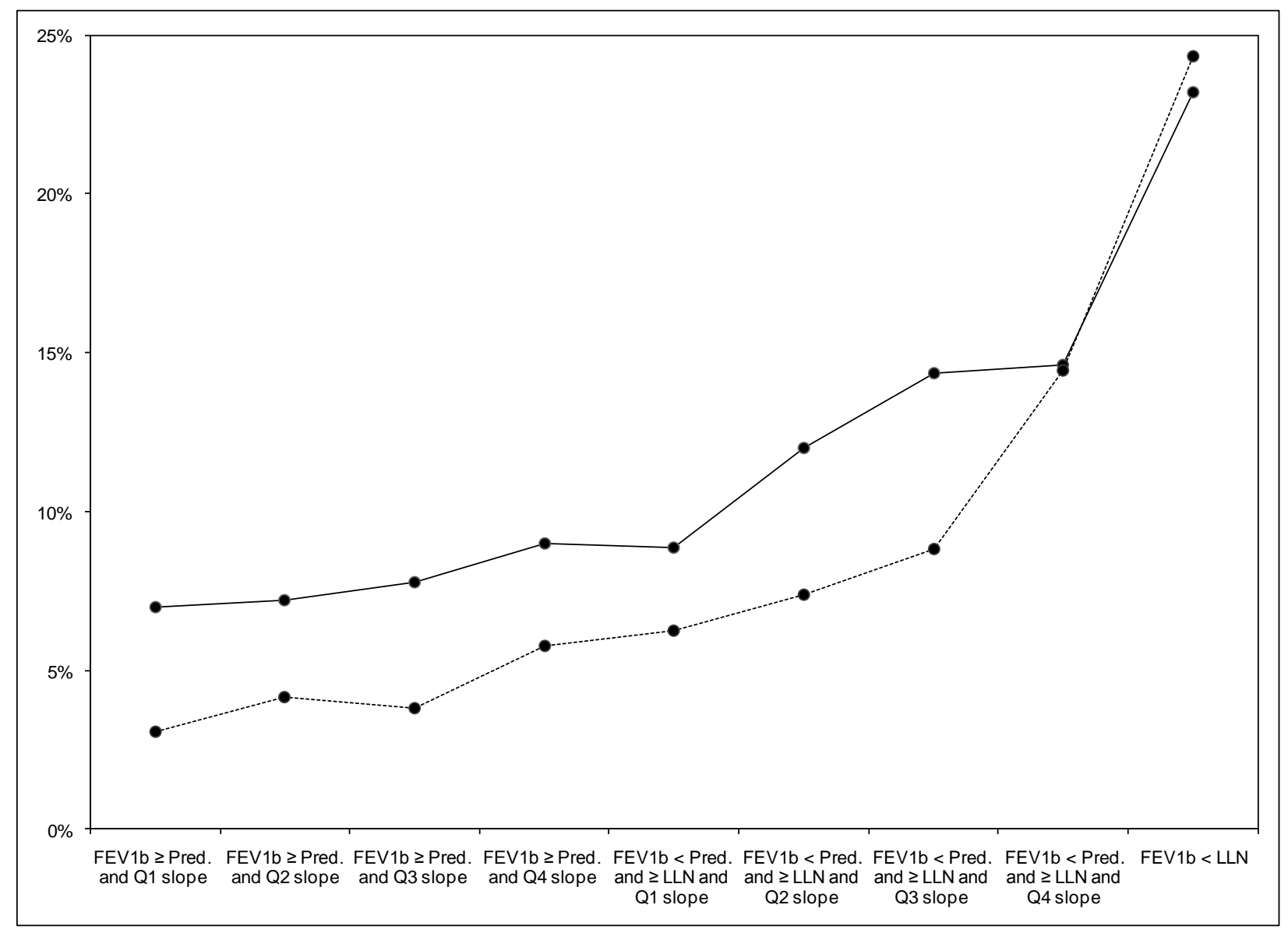

Figure 4.4 Overall prevalence rates of self-reported respiratory symptoms (chronic bronchitis = solid line; shortness of breath = dashed line) at examination two by lung function category. FEV ${ }_{1 b}$, baseline forced expiratory volume in one second; LLN, lower limit of normal; pred., predicted value. 


\begin{tabular}{|c|c|c|c|c|c|c|}
\hline & \multicolumn{2}{|c|}{ Males } & \multicolumn{2}{|c|}{ Females } & \multicolumn{2}{|c|}{ Never Smokers } \\
\hline & HR & $95 \% \mathrm{CI}$ & HR & $95 \% \mathrm{CI}$ & HR & $95 \%$ CI \\
\hline COPD Morbidity & \multicolumn{2}{|c|}{$(\mathrm{n}=4,550 ; \mathrm{COPD}=616)$} & \multicolumn{2}{|c|}{$(\mathrm{n}=5,833 ; \mathrm{COPD}=789)$} & \multicolumn{2}{|c|}{$(\mathrm{n}=1,943 ; \mathrm{COPD}=75$} \\
\hline $\mathrm{FEV}_{1 \mathrm{~b}} \geq$ pred. and Q1 slope & \multicolumn{2}{|c|}{1.00} & \multicolumn{2}{|c|}{1.00} & \multicolumn{2}{|c|}{1.00} \\
\hline $\mathrm{FEV}_{1 \mathrm{~b}} \geq$ pred. and Q2 slope & 0.68 & $(0.27-1.71)$ & 1.15 & $(0.42-3.15)$ & 0.27 & $(0.05-1.64)$ \\
\hline $\mathrm{FEV}_{1 \mathrm{~b}} \geq$ pred. and Q3 slope & 1.18 & $(0.54-2.58)$ & 1.52 & $(0.58-4.01)$ & 0.12 & $(0.01-1.12)$ \\
\hline $\mathrm{FEV}_{1 \mathrm{~b}} \geq$ pred. and Q4 slope & 1.38 & $(0.65-2.90)$ & 2.99 & $(1.20-7.44)$ & 0.68 & $(0.18-2.57)$ \\
\hline $\mathrm{FEV}_{1 \mathrm{~b}}<$ pred. and $\geq$ LLN and Q1 slope & 1.25 & $(0.60-2.60)$ & 3.68 & $(1.48-9.15)$ & 0.80 & $(0.21-3.10)$ \\
\hline $\mathrm{FEV}_{1 \mathrm{~b}}<$ pred. and $\geq$ LLN and Q2 slope & 1.85 & $(0.92-3.72)$ & 4.90 & $(2.00-11.97)$ & 1.15 & $(0.32-4.08)$ \\
\hline $\mathrm{FEV}_{1 \mathrm{~b}}<$ pred. and $\geq$ LLN and Q3 slope & 3.10 & $(1.56-6.17)$ & 6.06 & $(2.47-14.86)$ & 1.44 & $(0.41-5.11)$ \\
\hline $\mathrm{FEV}_{1 \mathrm{~b}}<$ pred. and $\geq$ LLN and Q4 slope & 5.11 & $(2.58-10.13)$ & 11.63 & $(4.75-28.46)$ & 3.09 & $(0.88-10.86)$ \\
\hline $\mathrm{FEV}_{1 \mathrm{~b}}<\mathrm{LLN}$ & 9.38 & $(4.82-18.24)$ & 18.05 & $(7.44-43.78)$ & 3.44 & $(1.00-11.77)$ \\
\hline COPD or CHD Mortality & \multicolumn{2}{|c|}{$(\mathrm{n}=4,598 ;$ Deaths $=1,053)$} & \multicolumn{2}{|c|}{$(\mathrm{n}=5,859 ;$ Deaths $=862)$} & \multicolumn{2}{|c|}{$(n=1,949 ;$ Deaths $=208) \dagger$} \\
\hline $\mathrm{FEV}_{1 \mathrm{~b}} \geq$ pred. and Q1 slope & \multicolumn{2}{|c|}{1.00} & \multicolumn{2}{|l|}{1.00} & \multicolumn{2}{|l|}{1.00} \\
\hline $\mathrm{FEV}_{1 \mathrm{~b}} \geq$ pred. and Q2 slope & 1.33 & $(0.77-2.31)$ & 1.93 & $(0.87-4.29)$ & 2.33 & $(0.68-7.91)$ \\
\hline $\mathrm{FEV}_{1 \mathrm{~b}} \geq$ pred. and Q3 slope & 1.23 & $(0.72-2.10)$ & 2.19 & $(0.99-4.84)$ & 2.39 & $(0.71-7.99)$ \\
\hline $\mathrm{FEV}_{1 \mathrm{~b}} \geq$ pred. and Q4 slope & 1.43 & $(0.85-2.39)$ & 3.04 & $(1.41-6.55)$ & 2.10 & $(0.64-6.90)$ \\
\hline $\mathrm{FEV}_{1 \mathrm{~b}}<$ pred. and $\geq$ LLN and Q1 slope & 1.48 & $(0.90-2.45)$ & 3.58 & $(1.65-7.75)$ & 2.92 & $(0.88-9.61)$ \\
\hline $\mathrm{FEV}_{1 \mathrm{~b}}<$ pred. and $\geq$ LLN and Q2 slope & 1.53 & $(0.93-2.50)$ & 3.46 & $(1.62-7.40)$ & 3.18 & $(0.98-10.33)$ \\
\hline $\mathrm{FEV}_{1 \mathrm{~b}}<$ pred. and $\geq$ LLN and Q3 slope & 1.96 & $(1.20-3.20)$ & 4.22 & $(1.97-9.05)$ & 2.78 & $(0.84-9.19)$ \\
\hline $\mathrm{FEV}_{1 \mathrm{~b}}<$ pred. and $\geq$ LLN and Q4 slope & 3.03 & $(1.86-4.95)$ & 7.47 & $(3.49-16.00)$ & 4.90 & $(1.48-16.30)$ \\
\hline $\mathrm{FEV}_{1 \mathrm{~b}}<\mathrm{LLN}$ & 3.71 & $(2.30-5.96)$ & 11.11 & $(5.22-23.62)$ & 4.50 & $(1.36-14.95)$ \\
\hline All-cause Mortality & \multicolumn{2}{|c|}{$(\mathrm{n}=4,598 ;$ Deaths $=3,217)$} & \multicolumn{2}{|c|}{$(\mathrm{n}=5,859 ;$ Deaths $=3,449)$} & \multicolumn{2}{|c|}{$(\mathrm{n}=1,949 ;$ Deaths $=987)$} \\
\hline $\mathrm{FEV}_{1 \mathrm{~b}} \geq$ pred. and Q1 slope & \multicolumn{2}{|l|}{1.00} & \multicolumn{2}{|l|}{1.00} & \multicolumn{2}{|l|}{1.00} \\
\hline $\mathrm{FEV}_{1 \mathrm{~b}} \geq$ pred. and Q2 slope & 1.03 & $(0.79-1.35)$ & 1.05 & $(0.81-1.37)$ & 1.05 & $(0.70-1.58)$ \\
\hline $\mathrm{FEV}_{1 \mathrm{~b}} \geq$ pred. and Q3 slope & 0.93 & $(0.72-1.21)$ & 1.16 & $(0.90-1.51)$ & 1.12 & $(0.76-1.66)$ \\
\hline $\mathrm{FEV}_{1 \mathrm{~b}} \geq$ pred. and Q4 slope & 1.12 & $(0.88-1.44)$ & 1.34 & $(1.05-1.72)$ & 1.22 & $(0.84-1.77)$ \\
\hline $\mathrm{FEV}_{1 \mathrm{~b}}<$ pred. and $\geq$ LLN and Q1 slope & 1.14 & $(0.90-1.45)$ & 1.30 & $(1.01-1.68)$ & 1.11 & $(0.75-1.64)$ \\
\hline $\mathrm{FEV}_{1 \mathrm{~b}}<$ pred. and $\geq$ LLN and Q2 slope & 1.34 & $(1.06-1.68)$ & 1.55 & $(1.22-1.98)$ & 1.38 & $(0.95-2.00)$ \\
\hline $\mathrm{FEV}_{1 \mathrm{~b}}<$ pred. and $\geq$ LLN and Q3 slope & 1.40 & $(1.11-1.77)$ & 1.62 & $(1.26-2.07)$ & 1.28 & $(0.88-1.88)$ \\
\hline $\mathrm{FEV}_{1 \mathrm{~b}}<$ pred. and $\geq$ LLN and Q4 slope & 2.01 & $(1.59-2.54)$ & 2.40 & $(1.87-3.08)$ & 1.89 & $(1.27-2.80)$ \\
\hline $\mathrm{FEV}_{1 \mathrm{~b}}<\mathrm{LLN}$ & 2.24 & $(1.79-2.80)$ & 2.85 & $(2.23-3.63)$ & 1.66 & $(1.12-2.47)$ \\
\hline
\end{tabular}

\footnotetext{
*Adjusted for baseline age and height.

$\dagger$ Sensitivity analysis was not possible due to a lack of cases in the reference category after excluding the adjusted $1981 \mathrm{FEV}_{1}$ values.

$\mathrm{FEV}_{1 \mathrm{~b}}$, baseline forced expiratory volume in one second; LLN, lower limit of normal; pred., predicted value.
} 


\begin{tabular}{|c|c|c|c|c|}
\hline & \multicolumn{2}{|c|}{ Baseline Age $\leq 45$} & \multicolumn{2}{|c|}{ Baseline Age $>45$} \\
\hline & HR & $95 \% \mathrm{CI}$ & HR & $95 \% \mathrm{CI}$ \\
\hline COPD Morbidity & \multicolumn{2}{|c|}{$(\mathrm{n}=2,726 ; \mathrm{COPD}=269)$} & \multicolumn{2}{|c|}{$(\mathrm{n}=7,657 ; \mathrm{COPD}=1,136)$} \\
\hline $\mathrm{FEV}_{1 \mathrm{~b}} \geq$ pred. and Q1 slope & 1.00 & & 1.00 & \\
\hline $\mathrm{FEV}_{1 \mathrm{~b}} \geq$ pred. and Q2 slope & 0.75 & $(0.23-2.47)$ & 0.91 & $(0.41-2.02)$ \\
\hline $\mathrm{FEV}_{1 \mathrm{~b}} \geq$ pred. and Q3 slope & 1.19 & $(0.41-3.43)$ & 1.35 & $(0.65-2.83)$ \\
\hline $\mathrm{FEV}_{1 \mathrm{~b}} \geq$ pred. and Q4 slope & 1.69 & $(0.63-4.50)$ & 2.15 & $(1.07-4.31)$ \\
\hline $\mathrm{FEV}_{1 \mathrm{~b}}<$ pred. and $\geq \mathrm{LLN}$ and Q1 slope & 1.92 & $(0.74-4.97)$ & 2.35 & $(1.17-4.71)$ \\
\hline $\mathrm{FEV}_{1 \mathrm{~b}}<$ pred. and $\geq \mathrm{LLN}$ and Q2 slope & 2.66 & $(1.06-6.71)$ & 3.29 & $(1.68-6.46)$ \\
\hline $\mathrm{FEV}_{1 \mathrm{~b}}<$ pred. and $\geq \mathrm{LLN}$ and Q3 slope & 2.64 & $(1.03-6.76)$ & 4.91 & $(2.51-9.60)$ \\
\hline $\mathrm{FEV}_{1 \mathrm{~b}}<$ pred. and $\geq \mathrm{LLN}$ and Q4 slope & 4.79 & $(1.88-12.17)$ & 8.48 & $(4.34-16.57)$ \\
\hline $\mathrm{FEV}_{1 \mathrm{~b}}<\mathrm{LLN}$ & 6.21 & $(2.52-15.33)$ & 15.26 & $(7.88-29.54)$ \\
\hline COPD or CHD Mortality & \multicolumn{2}{|c|}{$(\mathrm{n}=2,732 ;$ Deaths $=136)$} & \multicolumn{2}{|c|}{$(\mathrm{n}=7,725 ;$ Deaths $=1,779)$} \\
\hline $\mathrm{FEV}_{1 \mathrm{~b}} \geq$ pred. and Q1 slope & \multicolumn{2}{|c|}{1.00} & \multicolumn{2}{|c|}{1.00} \\
\hline $\mathrm{FEV}_{1 \mathrm{~b}} \geq$ pred. and Q2 slope & 1.12 & $(0.27-4.67)$ & 1.41 & $(0.88-2.27)$ \\
\hline $\mathrm{FEV}_{1 \mathrm{~b}} \geq$ pred. and Q3 slope & 1.69 & $(0.46-6.24)$ & 1.47 & $(0.93-2.35)$ \\
\hline $\mathrm{FEV}_{1 \mathrm{~b}} \geq$ pred. and Q4 slope & 0.86 & $(0.22-3.44)$ & 1.87 & $(1.19-2.91)$ \\
\hline $\mathrm{FEV}_{1 \mathrm{~b}}<$ pred. and $\geq$ LLN and Q1 slope & 1.71 & $(0.49-5.90)$ & 2.10 & $(1.35-3.28)$ \\
\hline $\mathrm{FEV}_{1 \mathrm{~b}}<$ pred. and $\geq$ LLN and Q2 slope & 2.67 & $(0.81-8.83)$ & 1.98 & $(1.28-3.07)$ \\
\hline $\mathrm{FEV}_{1 \mathrm{~b}}<$ pred. and $\geq$ LLN and Q3 slope & 1.59 & $(0.45-5.64)$ & 2.61 & $(1.69-4.03)$ \\
\hline $\mathrm{FEV}_{1 \mathrm{~b}}<$ pred. and $\geq \mathrm{LLN}$ and $\mathrm{Q} 4$ slope & 3.77 & $(1.12-12.71)$ & 4.09 & $(2.65-6.33)$ \\
\hline $\mathrm{FEV}_{1 \mathrm{~b}}<\mathrm{LLN}$ & 4.39 & $(1.36-14.23)$ & 5.46 & $(3.56-8.37)$ \\
\hline All-cause Mortality & \multicolumn{2}{|c|}{$(n=2,732 ;$ Deaths $=715)$} & \multicolumn{2}{|c|}{$(\mathrm{n}=7,725 ;$ Deaths $=5,951)$} \\
\hline $\mathrm{FEV}_{1 \mathrm{~b}} \geq$ pred. and Q1 slope & \multicolumn{2}{|l|}{1.00} & \multicolumn{2}{|l|}{1.00} \\
\hline $\mathrm{FEV}_{1 \mathrm{~b}} \geq$ pred. and Q2 slope & 1.17 & $(0.66-2.08)$ & 0.97 & $(0.80-1.19)$ \\
\hline $\mathrm{FEV}_{1 \mathrm{~b}} \geq$ pred. and Q3 slope & 1.55 & $(0.90-2.66)$ & 0.99 & $(0.82-1.21)$ \\
\hline $\mathrm{FEV}_{1 \mathrm{~b}} \geq$ pred. and Q4 slope & 1.62 & $(0.96-2.72)$ & 1.14 & $(0.95-1.37)$ \\
\hline $\mathrm{FEV}_{1 \mathrm{~b}}<$ pred. and $\geq$ LLN and Q1 slope & 1.69 & $(1.02-2.81)$ & 1.15 & $(0.95-1.38)$ \\
\hline $\mathrm{FEV}_{1 \mathrm{~b}}<$ pred. and $\geq$ LLN and Q2 slope & 2.00 & $(1.22-3.29)$ & 1.35 & $(1.13-1.61)$ \\
\hline $\mathrm{FEV}_{1 \mathrm{~b}}<$ pred. and $\geq$ LLN and Q3 slope & 1.81 & $(1.09-3.02)$ & 1.42 & $(1.19-1.70)$ \\
\hline $\mathrm{FEV}_{1 \mathrm{~b}}<$ pred. and $\geq \mathrm{LLN}$ and Q4 slope & 2.66 & $(1.60-4.43)$ & 2.06 & $(1.72-2.47)$ \\
\hline $\mathrm{FEV}_{1 \mathrm{~b}}<\mathrm{LLN}$ & 3.21 & $(1.98-5.23)$ & 2.35 & $(1.97-2.80)$ \\
\hline
\end{tabular}

*Models adjusted for baseline age and height.

$\mathrm{FEV}_{1 \mathrm{~b}}$, baseline forced expiratory volume in one second; LLN, lower limit of normal; pred., predicted value. 


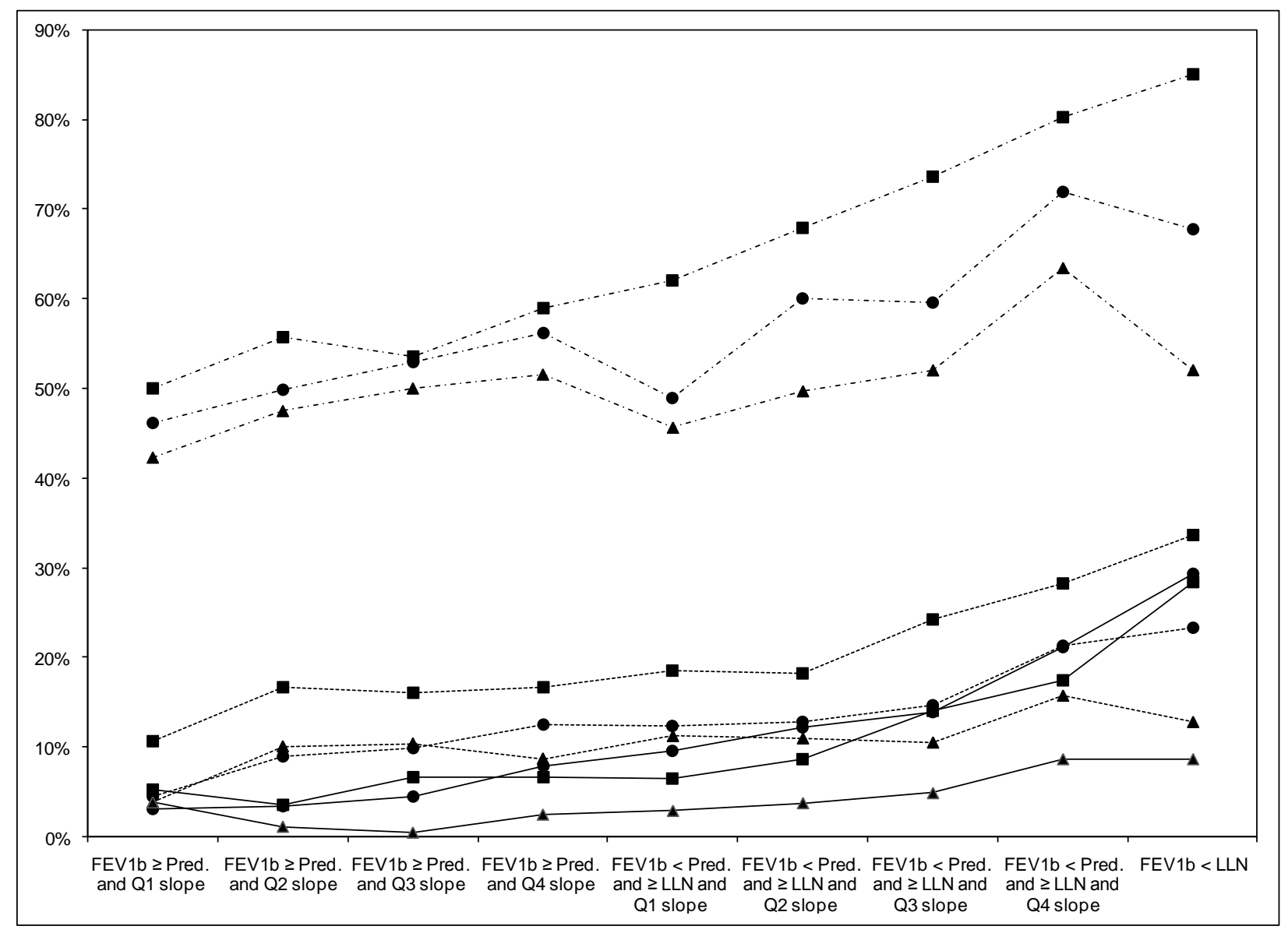

Figure 4.5 Cumulative incidence by lung function category, for three subcohorts $(\boldsymbol{\bullet}=$ males; $\bullet=$ females; $\boldsymbol{\Delta}=$ never smokers $)$ and three outcomes (COPD morbidity $=$ solid line; COPD or CHD mortality = dashed line; all-cause mortality $=$ dash dot line). $F E V_{1 b}$, baseline forced expiratory volume in one second; LLN, lower limit of normal; pred., predicted value. 
Chapter 5 


\section{Chapter 5}

\subsection{Summary}

Excessive lung function decline is an indicator of an underlying adverse health condition that may lead to the development of COPD. The principal risk factor for COPD is tobacco smoking, a highly-prevalent risk factor in the Copenhagen City Heart Study sample used for this study. ${ }^{69,70}$ Accordingly, this was a fitting sample for the current study because a majority of subjects were at risk for lung function decline. Previous literature on lung function decline has focused on characterizing lung function decline as an independent risk factor for morbidity and mortality, through the use of multivariate models adjusted for known risk factors. ${ }^{24-34,39,54-57}$ Although the risk of mortality has been studied most often, these studies demonstrate that lung function decline is a risk factor for morbidity and mortality. This earlier research provides the basis for the current study, one that followed a different approach to investigate lung function as a predictor of morbidity and mortality rather than as a causative factor among a multitude of other risk factors.

Morbidity and mortality risks associated with lung function decline in minimally-adjusted models are less often studied. However, there is a need to understand the relationships between the outcome of lung function decline with morbidity and mortality to help inform prevention activities that are based on observed lung function decline. Although longitudinal spirometry is conducted in clinical and occupational settings, the evaluation of the rate of decline in individuals in prospectively collected spirometry data is a subject of current research, 5,6 including such topics as how soon one can make sound decisions for prevention and intervention based upon these data. This study helped to address questions about the usefulness of periodic 
spirometry and screening criteria to make optimal use of periodic spirometry for disease prevention and intervention measures.

Specifically, the goal of this study was to evaluate the epidemiologic nature and utility of longitudinal measurements of $\mathrm{FEV}_{1}$ as predictors for COPD, COPD or CHD mortality, and allcause mortality in an aging, population-based sample. The results of this evaluation were intended to be applicable to community and workplace screening of at-risk individuals for respiratory disease and in the secondary prevention of disease. By design, the minimally-adjusted models were intended to maximize the observed effects of the respiratory health status outcomes (lung function level and rate of decline, respiratory symptoms, and asthma) on morbidity and mortality risks, as each can be used as indicators for prevention purposes. Inclusion of other possible risk factors, which often contribute to these respiratory health status outcomes, would have partially accounted for the effects of these outcomes and detracted from our study's purpose and the relationships of interest.

Three related studies accomplished various objectives related to the overall goal of this project. The first study addressed questions pertaining to methods used in occupational respiratory health monitoring programs, namely the value of longitudinal spirometry data as compared to selfreported respiratory symptom questionnaire data, and various representations and methods of assessing longitudinal spirometry data. ${ }^{51,52}$ The second study benefited from the agestratification of the sample, by examining morbidity and mortality risks among individuals below ages when spirometry is recommended and COPD typically becomes apparent. ${ }^{88,89}$ The second study also benefited from the necessary mid-study change in model of spirometer, investigating risks associated with patterns of decline created from the two periods. Building upon the 
observed associations between the level of lung function and the rate of decline with morbidity and mortality risks in the first two studies, a novel and more direct assessment was made with the combination of the level and the rate of decline as a predictor of morbidity and mortality.

Results from the first study demonstrated the predictive capacities of four criteria for lung function decline potentially usable in practice.$^{51,52}$ Among the quartile-based criteria, higher morbidity and mortality risks were associated with the slope than with the relative slope. As for the two limits of longitudinal decline, higher morbidity and mortality risks were associated with the ACOEM LNL than with the $90 \mathrm{ml} / \mathrm{yr}$ limit. In general, associations were strongest with COPD morbidity outcomes. Associations with COPD or CHD mortality were slightly higher than with all-cause mortality. Females were at greater risk than males across all measures of lung function decline, as found in the literature. ${ }^{75}$ With lower tobacco consumption than males, this may indicate higher susceptibility in females. ${ }^{76,77}$ Never smokers had increasing trends overall, but their trends were less often statistically significant.

Critical rates of decline associated with increased morbidity risk were observed at the third quartiles of the slope (declines starting at $60 \mathrm{ml} / \mathrm{yr}$ for males and $57 \mathrm{ml} / \mathrm{yr}$ for females) with risks greater than 2.5 times those for the reference. For mortality, the fourth quartiles of the slope (declines starting at $118 \mathrm{ml} / \mathrm{yr}$ for males and $95 \mathrm{ml} / \mathrm{yr}$ for females) nearly doubled COPD or CHD mortality risks for males and tripled them for females. Respective risks for all-cause mortality increased by $67 \%$ and $88 \%$. Similarities exist with community-based and occupational studies of lung function decline and respiratory morbidity and mortality, ${ }^{29,33,36,57,68}$ including slightly higher risks in females as compared to males for COPD hospitalization and all-cause mortality ${ }^{29,33}$ and increased risk of cardiac mortality in males. ${ }^{57}$ Similarities were also found in a 
study of underground coal miners with a two-fold cardiovascular and nonmalignant respiratory disease mortality risk, ${ }^{36}$ and statistically significant declines above $90 \mathrm{ml} / \mathrm{yr}$ for mortality as were declines of $60 \mathrm{ml} / \mathrm{yr}$ or more for never smokers. ${ }^{68}$

Lung function decline demonstrated often greater risks than respiratory symptoms and asthma, but their risks remained statistically significant in multivariate models with lung function. Reported respiratory symptoms (chronic bronchitis, cough, phlegm, and shortness of breath) and asthma have been associated with increased morbidity and mortality risks, ${ }^{78-82}$ even after controlling for $\mathrm{FEV}_{1}{ }^{83}$ In contrast, the current study did not identify increased risk between asthma and respiratory mortality, but this may be due to our specific focus on COPD. ${ }^{78}$ Additionally, chronic bronchitis was often a significant mortality predictor in our study, even after adjustment for shortness of breath and height-adjusted baseline lung function. ${ }^{83}$

In the second study's reassessment of critical cut-points where lung function decline was associated with increased morbidity and mortality risks using spirometry data from the last two examinations and extended outcome follow-up, the results were generally consistent with our previous assessment from examinations one and two, despite the adjustment to the $1981 \mathrm{FEV}_{1}$ values in the first study and possible influences of improvements in spirometry standards. High COPD morbidity and mortality risks were again associated with lower lung function levels and excessive decline. Morbidity and mortality risks increased starting at -77 and $-54 \mathrm{ml} / \mathrm{yr}$ in males and -57 and $-41 \mathrm{ml} / \mathrm{yr}$ in females, respectively. A cut-point in the $\mathrm{FEV}_{1}$ slope of $-49 \mathrm{ml} / \mathrm{yr}$ was associated with the start of increased morbidity and mortality risks in the overall cohort. General similarities to previous studies include significant associations between lung function decline and COPD hospitalizations and mortality, with some studies reporting higher associations for 
females than for males. ${ }^{27-29,33}$ Again, $\mathrm{FEV}_{1}$ slope cut-points demonstrating increased mortality risks in males were similar to those for underground coal miners, risks that increased at -60 $\mathrm{ml} / \mathrm{yr}$ and were statistically significant at $-90 \mathrm{ml} / \mathrm{yr}^{68}$

For individuals with a baseline age of 45 years or younger, excessive decline was a significant predictor of morbidity and mortality. These results are relevant to screening practices as spirometry testing is currently recommended for individuals age 45 or older with other risk factors. ${ }^{88,89}$ Further, considering gender and age, morbidity and mortality risks were higher among females, even with lower rates of smoking in younger females. ${ }^{76,77}$ As for symptoms, chronic bronchitis was a significant predictor of morbidity in younger individuals, as in the Framingham Offspring cohort. ${ }^{96}$ Given these results for younger individuals, and that low lung function in young, non-asthmatic adults predicts airflow obstruction at middle age, ${ }^{97}$ screening at earlier ages in at-risk populations (e.g., smokers, symptomatic individuals, occupationallyexposed groups) may be useful. Additional evidence for early screening resulted from the estimation of risks associated with early, late, and persistent patterns of lung function decline, where increasing morbidity and mortality risks were observed with increasing persistence of excessive decline and respiratory symptoms in males and females. Never smokers had a two-fold mortality risk with persistent excessive decline. Screening for early declines as these occurring over 4-7 years followed by intervention could help prevent the even greater risks observed with late or persistent declines.

The novel approach offered by the third study in the estimation of morbidity and mortality risks associated with lung function, whereby categories were created from the level and rate of lung function decline, showed one central and important finding. An excessive rate of decline is 
associated with increased morbidity and mortality risks, even with a 'normal' baseline level of lung function. Increasing trends in risk were consistently identified with baseline $\mathrm{FEV}_{1}$ at or above the LLN and increasing quartiles of decline across males, females, never smokers, and with a baseline age above and below 45 . Further, population attributable risks ${ }^{112}$ for an abnormal $\mathrm{FEV}_{1}$ (below the LLN), and then jointly with the category of a 'normal' $\mathrm{FEV}_{1}$ and the steepest declines demonstrated substantial additional contributions, indicating that accelerated $\mathrm{FEV}_{1}$ decline in those with 'normal' $\mathrm{FEV}_{1}$ is of public health significance and concern. Broad similarities to prior studies were higher morbidity and mortality risks among females, ${ }^{29,33}$ which may result from increased susceptibility to the effects of smoking, ${ }^{77}$ and increased COPD morbidity risks with lower lung function at younger ages. ${ }^{96}$

However, chronic inflammatory processes in the lungs that lead to elastic tissue loss and small airways remodeling with $\mathrm{COPD}^{105}$ progress over many years before disease can be detected. This inflammation in the lungs may also contribute to systemic inflammation and non-pulmonary disease. ${ }^{108}$ The consequences of a single abnormal spirometry test result differs for individuals, with some following a normal age trajectory, and others with normal results can have excessive decline. Early COPD detection may be improved through longitudinal spirometry testing in atrisk populations. ${ }^{21,98}$ Well-established risk factors for excessive decline are similar to those for COPD. ${ }^{1,33,34,96}$ Longitudinal spirometry may identify those individuals who have a high level of lung function but excessive decline, whose disease could progress undetected leading to increased morbidity and mortality risks. ${ }^{51,109}$

In summary, although it is well-know that utilization of longitudinal spirometry data is often complex due to issues of data standardization and quality, and effective interpretation of 
results, ${ }^{109}$ solutions have been offered to help overcome these challenges. ${ }^{113,114}$ This study acknowledges these challenges. Nonetheless, it also offers new knowledge to help advance research on lung function decline related to prevention of lung function decline, intervention on excessive lung function decline, secondary disease prevention, and reduction of premature mortality.

\subsection{Significance}

The goal of this study was to evaluate the epidemiologic nature and utility of longitudinal lung function measurements as predictors for COPD, COPD or CHD mortality, and all-cause mortality in minimally-adjusted models using an at-risk, aging, population-based sample for the purposes of improving disease prevention. The significance of this study lies not only in the estimation of the morbidity and mortality risks from a long outcome follow-up, but also in the applicability of the results for prevention. The first study compared multiple measures of longitudinal lung function decline $\left(\mathrm{FEV}_{1}\right.$ slope, $\mathrm{FEV}_{1}$ relative slope, $\mathrm{ACOEM} \mathrm{LNL}$, and a 90 $\mathrm{ml} / \mathrm{yr}$ limit), adjusted for respiratory symptoms and asthma, as predictors of morbidity and mortality. The logic behind these models was correspondence to data resulting from occupational respiratory monitoring programs and to demonstrate the relative value of the longitudinal measures and respiratory symptoms and asthma, and also the relative value of longitudinal and cross-sectional evaluation of spirometry data. The second study focused mainly on estimating risks for individuals younger than the recommended age for spirometry testing ${ }^{107}$ and for patterns of early, late, and persistent lung function decline. The message of this study was that earlier intervention may be beneficial in preventing the incidence of COPD morbidity and premature mortality. The third study involved a novel method of combining the level of lung function and the rate of decline to study their combined effect as a predictor of morbidity and mortality. These 
results may be very important. Significantly increased morbidity and mortality risks were observed for individuals with a normal level of lung function and a steep decline, demonstrating that use of only a cross-sectional comparison of the lung function level to a reference population to determine normality would fail to identify a number of individuals at increased risk for morbidity and mortality.

The significance of this study is relevant to healthcare practitioners, including both primary care practitioners with regard to recognition of persistent symptoms or exposures in patients, uses of spirometry, and timely referral for specialized care; and specialized care providers where the extent of the problem is determined with spirometry and a range of the patients are treated, from individuals with early signs of disease to those whose existing disease is managed and monitored for progression. This study is also relevant to workplace prevention where respiratory health screening and monitoring are conducted due to potentially hazardous exposures, and to public health through three broad Healthy People 2020 Respiratory Disease, COPD objectives: RD-10 (Reduce deaths from COPD among adults.), RD-11 (Reduce hospitalizations for COPD.), and RD-13 ([Developmental] Increase the proportion of adults with abnormal lung function whose underlying obstructive disease has been diagnosed.). ${ }^{49}$

\subsection{Strengths and Limitations}

The use of a large, age-stratified, population-based sample with a prospective study design and long outcome follow-up demonstrate significant strengths in the three studies. The high prevalence rates of smoking provided an excellent sample in which to study morbidity and mortality risks associated with lung function decline. Given that this is an at-risk sample, the

findings are relevant to both clinical and workplace disease prevention and cost-reduction. ${ }^{66,67}$ 
There are several limitations shared by the three studies. As described in the first study, preliminary analysis of mean $\mathrm{FEV}_{1}$ values by year of examination revealed a slightly excessive increase in mean $\mathrm{FEV}_{1}$ in $1981(\mathrm{n}=4,319)$, but not in $1982(\mathrm{n}=5,353)$ or $1983(\mathrm{n}=2,593)$, as compared to mean $\mathrm{FEV}_{1}$ at the baseline examinations in 1976-78. An a priori decision was made to devise a method of adjustment using a mean value, a generally statistically reasonable approach, in order to adjust and retain the 1981 data. The decision to adjust rather than to exclude the 1981 values was based on the potential loss of subjects and a reduction in power if these data were excluded. More importantly, we suspected COPD morbidity outcomes would be few in number for never smokers. Sensitivity analyses were conducted to assess the effect of the adjustment on the results from models that used the adjusted data values, whereby the Cox models were conducted on samples excluding the adjusted 1981 values (Appendices A-C).

Results of the sensitivity analysis for the first study indicated that the risks did not appear to be overestimated in the models with the adjusted data. The inclusion of the adjusted data resulted in an underestimation of the COPD morbidity risks for the level of lung function, the slope, and the relative slope and mainly so for never smokers and more often for females than for males as well as underestimation of COPD or CHD mortality risk for the level of lung function for never smokers. In the second study, the adjustment appeared to have resulted in an underestimation of risks, including the fourth quartiles of the slope for COPD morbidity risk in males over age 45 and for COPD or CHD mortality risk in females age 45 years and younger, and with a persistent pattern of decline in the slope for COPD morbidity risk in males. For the third study, sensitivity analyses indicated under- and overestimation of COPD morbidity and COPD or CHD mortality risks, where overestimation was found for COPD morbidity risk in never smokers and COPD or CHD mortality risk in individuals with a baseline age of 45 years and younger. 
The spirometry measurements were collected across a 28-year span. However, the necessary change in the model of spirometer after examination two, due to mechanical failure, ${ }^{98}$ prevented estimation of morbidity and mortality risks associated with lung function decline across the entire study period. Thus, only two spirometry measurements were used to calculate the rate of change in $\mathrm{FEV}_{1}$, creating a vulnerability to measurement error, but the use of quartile groupings was used to address this bias and could have reduced this effect, if the error was not excessive.

These studies were not designed to account for possible mixing between cause and effect with regard to steeply declining lung function and COPD outcomes. Nor did they adjust for many possible confounders. The research question was purely in the relationships of lung function decline with morbidity and mortality for prevention purposes rather than causation. For example, individuals with abnormal baseline lung function were included in the models for the third study, but post hoc analyses excluding these individuals showed little difference from the results of the original models. Lung function decline was a significant predictor of morbidity and mortality in either scenario.

The $90 \mathrm{ml} / \mathrm{yr}$ limit of decline used in the first study was from research on males. ${ }^{52}$ This limit was used for females as well, because no corresponding limit was found in the literature for females. Even though females generally have smaller lungs, ${ }^{75}$ in this at-risk population, the $90 \mathrm{ml} / \mathrm{yr}$ limit was appropriate as it was similar to the fourth quartile of the slope in females, starting at 95 $\mathrm{ml} / \mathrm{yr}$.

Two possible sources of bias in the outcomes are underdiagnosis of COPD and underreporting of COPD as a cause of death. These could have resulted in misclassification, biasing the results toward the null and decreasing the associations. ${ }^{84}$ This potential bias was addressed by 
combining the often interrelated COPD and CHD mortality into one cardiopulmonary mortality outcome. $^{60,61,102}$ Also, as COPD morbidity was defined with a hospital diagnosis, our results generally represent risks for individuals with more severe disease. Generalizability of the results may be limited to individuals with more severe disease, but also likely the individuals representing the highest costs and in need of the most care.

Other possible selection bias includes subjects lost to follow-up who were older at baseline, had a lower lung function level, and a higher proportion of self-reported current asthma, respiratory symptoms, and smoking as compared those who were included in the studies due to participation and spirometry testing at examinations one and two and/or examinations three and four. This bias could have caused an underestimation of risk, particularly in the second study with the estimation of risks associated with patterns of lung function decline using participants attending all four examinations. Risks of morbidity and mortality could also have been underestimated due to self-selection of healthier subjects into the Copenhagen City Heart Study.

\subsection{Future Research}

The findings of this study identify the need for further investigation of gender differences in morbidity and mortality risks associated with the level and rate of lung function decline and respiratory symptoms and asthma. Gender differences related to COPD are not fully clear or understood. $^{77,115}$ Additional measures of lung function could be considered in future research. This study focused upon $\mathrm{FEV}_{1}$ as it has been identified as the best lung function measurement to estimate the rate of decline, ${ }^{71}$ but recent research has reconsidered the value of $F E V_{1}$ in comparison to $\mathrm{FVC}$ and the ratio of $\mathrm{FEV}_{1} / \mathrm{FVC}$ in predicting survival. ${ }^{116}$ Additional analyses could be conducted using these two alternative measures to determine their value for prevention 
purposes in the context of the models investigated in this study. Other population-based data sets, such as the NHANES, could be used to assess whether the results of this study could be replicated or expanded upon using other populations. Finally, understanding the practical value and applicability of this research, closing a gap between research and practice, is of utmost importance. As there are barriers to the implementation of longitudinal spirometry such as cost, data standardization and quality, and effective interpretation of results, ${ }^{109}$ studies could be conducted pertaining to the feasibility, performance, and individual benefit of implementing longitudinal spirometry in healthcare and occupational settings, including evaluation of new and existing programs.

\subsection{Conclusion}

The findings of this study extend knowledge on lung function as a predictor of adverse health outcomes in an aging population, the need for early identification and prevention of lung function decline, and the possibilities to decrease associated risks for COPD morbidity and premature mortality in individuals who have significant exposures to known risk factors for lung function decline or the development of COPD. The results of this study build upon previous findings of lung function decline as a risk factor for adverse outcomes. They also add to our understanding of the epidemiologic nature and utility of lung function decline as a predictor. Results especially pertinent to workplace respiratory monitoring programs are that the level and rate of lung function decline generally demonstrated higher morbidity and mortality risks than respiratory symptoms and asthma. Comparison of the risks associated with the level and rate of lung function decline provided further evidence that evaluation of lung function decline is important in disease prevention. 
From a clinical and public health perspective, associations between lung function decline and increased morbidity and mortality risks in individuals at younger ages provided evidence that lung function testing before middle age may be a worthwhile investment. This could represent opportunities for early intervention and preservation of lung function. The risks associated with early, late, and persistent patterns of lung function decline lend further support that early detection of lung function decline coupled with appropriate intervention may reduce morbidity and mortality risks.

Estimates of the combined effect of the level of lung function and the rate of lung function decline as a predictor of morbidity and mortality further demonstrated that a combined approach to lung function assessment may provide more useful information for prevention and early identification of disease. This analysis also revealed the importance of monitoring for lung function decline even in individuals with a 'normal' level of lung function. The findings suggest that not only is lung function decline an important predictor of morbidity and mortality, but also that early monitoring may be beneficial in reducing the burden of COPD morbidity and mortality and premature mortality. Future research using other population-based studies may further elucidate the relationship of lung function decline with morbidity and mortality and how to translate results into practical applications that improve the effectiveness of spirometry monitoring and risk reduction in clinical and occupational settings. 
Appendix A: Sensitivity Analyses for Study 1 


\begin{tabular}{|ll}
$\begin{array}{l}\text { Examination 1 } \\
1976-78\end{array}$ & $\begin{array}{l}\text { Examination } 2 \\
1982-83\end{array}$ \\
13,826 Subjects & $\begin{array}{l}\text { End of Follow-up } \\
2,895 \text { Subjects }\end{array}$ \\
\hline $\begin{array}{l}\text { Study cohort: } \\
\text { 6,213 Subjects }\end{array}$ & $\begin{array}{l}\text { Spirometry and ascertainment } \\
\text { of asthma, chronic bronchitis, } \\
\text { and shortness of breath }\end{array}$ \\
\hline
\end{tabular}

Figure A.1 Sample size and morbidity and mortality follow-up. Above the timeline are the numbers of subjects who participated in spirometry testing by Copenhagen City Heart Study examination. Below the timeline is the number of subjects who participated in spirometry testing at examinations one and two and the numbers of health outcomes that occurred during the morbidity and mortality follow-up periods. Note that the follow-up periods are represented as dashed lines. COPD morbidity follow-up lasted until 2003. COPD or CHD mortality follow-up lasted until 2006. All-cause mortality follow-up lasted until 2007. 


\begin{tabular}{|c|c|c|c|c|c|c|}
\hline \multirow{2}{*}{$\begin{array}{l}\text { Data for lung function level } \\
\text { (Examination two) } \\
\text { Subjects }(\mathrm{n}, \%)^{*}\end{array}$} & \multicolumn{2}{|c|}{ Males } & \multicolumn{2}{|c|}{ Females } & \multicolumn{2}{|c|}{ Never smokers } \\
\hline & 3,542 & 44.6 & 4,404 & 55.4 & 1,669 & 21.0 \\
\hline Age at examination two (mean, $\mathrm{SD}_{\dagger}^{\dagger}$ ) & 56.3 & 12.5 & 56.7 & 12.0 & 56.5 & 13.7 \\
\hline \multicolumn{7}{|l|}{ Follow-up, years (mean, SD) } \\
\hline COPD morbidity & 14.9 & 7.0 & 17.0 & 6.0 & 17.9 & 5.6 \\
\hline COPD or CHD mortality & 16.7 & 7.8 & 19.0 & 6.8 & 19.8 & 6.5 \\
\hline All-cause mortality & 16.9 & 8.0 & 19.3 & 7.0 & 20.2 & 6.7 \\
\hline Height, $\mathrm{cm} \dagger$ (mean, SD) & 174 & 7 & 161 & 6 & 165 & 9 \\
\hline $\mathrm{FEV}_{1}, 1 \dagger($ mean, $\mathrm{SD})$ & 2.78 & 0.87 & 2.01 & 0.60 & 2.37 & 0.83 \\
\hline $\mathrm{FEV}_{1} /$ height $^{2} \mathrm{x}$ mean height ${ }^{2}, 1$ (mean, SD) & 2.54 & 0.72 & 2.13 & 0.58 & 2.39 & 0.66 \\
\hline 25th percentile & 2.07 & & 1.76 & & $2.40 ; 1.87 \ddagger$ & \\
\hline Median & 2.55 & & 2.12 & & $2.90 ; 2.20 \ddagger$ & \\
\hline 75th percentile & 3.03 & & 2.51 & & $3.36 ; 2.60 \%$ & \\
\hline Asthma (n, \%) & 113 & 3.2 & 152 & 3.5 & 35 & 2.1 \\
\hline Chronic bronchitis (n, \%) & 570 & 16.1 & 491 & 11.2 & 99 & 5.9 \\
\hline Shortness of breath (n, \%) & 349 & 9.9 & 483 & 11.0 & 103 & 6.2 \\
\hline Never smokers (n, \%) & 424 & 12.0 & 1,245 & 28.3 & $424 ; 1,245 \ddagger$ & $25.4 ; 74.6 \%$ \\
\hline Former smokers (n, \%) & 880 & 24.8 & 788 & 17.9 & & \\
\hline Current smokers (n, \%) & 2,237 & 63.2 & 2,366 & 53.7 & & \\
\hline \multicolumn{7}{|l|}{$\begin{array}{l}\text { Data for lung function decline } \\
\text { (Examination one to two }) \S\end{array}$} \\
\hline Subjects $(\mathrm{n}, * \%)$ & 2,716 & 43.7 & 3,497 & 56.3 & 1,172 & 18.9 \\
\hline Baseline age (mean, SD) & 52.7 & 11.4 & 52.7 & 10.9 & 52.9 & 12.2 \\
\hline \multicolumn{7}{|l|}{ Follow-up, years (mean, SD) } \\
\hline COPD morbidity & 15.0 & 6.8 & 17.1 & 5.8 & 18.0 & 5.4 \\
\hline COPD or CHD mortality & 16.7 & 8.0 & 19.0 & 6.8 & 19.9 & 6.5 \\
\hline All-cause mortality & 16.5 & 7.8 & 19.3 & 7.0 & 20.2 & 6.7 \\
\hline Baseline $\mathrm{FEV}_{1}, 1$ (mean, SD) & 3.09 & 0.82 & 2.24 & 0.56 & 2.57 & 0.80 \\
\hline Baseline $\mathrm{FEV}_{1} /$ height $^{2} \mathrm{x}$ mean height ${ }^{2}, 1$ (mean, SD) & 2.83 & 0.67 & 2.39 & 0.54 & 2.62 & 0.63 \\
\hline Slope $\mathrm{FEV}_{1}, \mathrm{ml} / \mathrm{yr} \dagger$ (mean, SD) & -69 & 91 & -51 & 66 & -50 & 77 \\
\hline 25th percentile & -132 & & -95 & & $-116 ;-95 \ddagger$ & \\
\hline Median & -76 & & -57 & & $-58 ;-57 \dagger$ & \\
\hline 75th percentile & -19 & & -19 & & $0 ; 0 \%$ & \\
\hline Slope $\mathrm{FEV}_{1} /$ baseline $\mathrm{FEV}_{1}, \% / \mathrm{yr}$ (mean, SD) & -2.2 & 3.3 & -2.2 & 3.2 & -1.8 & 3.3 \\
\hline 25th percentile & -4.1 & & -4.2 & & $-3.5 ;-3.9 \ddagger$ & \\
\hline Median & -2.4 & & -2.4 & & $-1.7 ;-2.2 \ddagger$ & \\
\hline 75th percentile & -0.6 & & -0.7 & & $0.0 ; 0.0 \ddagger$ & \\
\hline $\mathrm{FEV}_{1}$ below $\mathrm{LNL} \dagger(\mathrm{n}, \%)$ & 442 & 16.3 & 593 & 17.0 & 239 & 20.4 \\
\hline $\mathrm{FEV}_{1}$ decline of $90 \mathrm{ml} / \mathrm{yr}$ or more & 1,174 & 43.2 & 1,021 & 29.2 & 358 & 30.6 \\
\hline Asthma (n, \%) & 80 & 3.0 & 120 & 3.4 & 25 & 2.1 \\
\hline Chronic bronchitis (n, \%) & 434 & 16.0 & 380 & 10.9 & 64 & 5.5 \\
\hline Shortness of breath (n, \%) & 257 & 9.5 & 361 & 10.3 & 68 & 5.8 \\
\hline
\end{tabular}

*A total of 7,946 subjects were present for examination two and 6,213 subjects were present for examinations one and two. $\dagger \mathrm{SD}$ (standard deviation), cm (centimeters), 1 (liters), ml/yr (milliliters/year), and LNL (Longitudinal Normal Limit).

$\$$ Males; females.

$\S$ Includes subjects with approximately five years between spirometry tests. 


\begin{tabular}{|c|c|c|c|c|c|c|}
\hline \multirow{3}{*}{$\begin{array}{l}\text { Data for lung function level* } \\
\text { (Examination two) }\end{array}$} & \multicolumn{2}{|c|}{ Males } & \multirow{2}{*}{\multicolumn{2}{|c|}{$\begin{array}{c}\text { Females } \\
(\mathrm{n}=4,376 ; \text { COPD }=458)\end{array}$}} & \multirow{2}{*}{\multicolumn{2}{|c|}{$\begin{array}{c}\text { Never smokers } \\
(\mathrm{n}=1,665 ; \mathrm{COPD}=54)\end{array}$}} \\
\hline & \multicolumn{2}{|c|}{$(\mathrm{n}=3,506 ; \mathrm{COPD}=378)$} & & & & \\
\hline & HR & $95 \%$ CI & HR & $95 \% \mathrm{CI}$ & HR & \multirow[t]{2}{*}{$95 \% \mathrm{CI}$} \\
\hline Q1 FEV $1 /$ height $^{2}$ & \multicolumn{2}{|l|}{1.00} & \multicolumn{2}{|l|}{1.00} & 1.00 & \\
\hline Q2 $\mathrm{FEV}_{1} /$ height $^{2}$ & 2.95 & $(1.73-5.04)$ & 2.48 & $(1.56-3.93)$ & 2.12 & $(0.54-8.32)$ \\
\hline Q3 FEV Feight $^{2}$ & 6.71 & $(3.99-11.27)$ & 5.86 & $(3.77-9.12)$ & 2.33 & $(0.60-9.10)$ \\
\hline Asthma $\dagger$ & 2.03 & $(1.44-2.85)$ & 1.32 & $(0.95-1.85)$ & 4.13 & $(1.67-10.17)$ \\
\hline Chronic bronchitis $\dagger$ & 2.11 & $(1.67-2.67)$ & 2.32 & $(1.86-2.89)$ & 3.52 & $(1.78-6.99)$ \\
\hline Shortness of breath $\dagger$ & 2.55 & $(1.95-3.33)$ & 2.58 & $(2.04-3.27)$ & 2.36 & $(1.08-5.15)$ \\
\hline Data for lung function decline & \multicolumn{2}{|c|}{$(\mathrm{n}=2,683 ; \mathrm{COPD}=289)$} & \multicolumn{2}{|c|}{$(\mathrm{n}=3,448 ; \mathrm{COPD}=356)$} & \multicolumn{2}{|c|}{$(\mathrm{n}=1,162 ; \mathrm{COPD}=37)$} \\
\hline (Examination one to two) & HR & $95 \% \mathrm{CI}$ & HR & $95 \% \mathrm{CI}$ & HR & $95 \%$ CI \\
\hline Q4 FEV ${ }_{1}$ slope & 4.13 & $(2.83-6.03)$ & 7.07 & $(4.97-10.06)$ & 5.46 & $(1.31-22.74)$ \\
\hline Asthma & 1.30 & $(0.86-1.96)$ & 0.85 & $(0.57-1.27)$ & 4.60 & $(1.23-17.28)$ \\
\hline Chronic bronchitis & 1.79 & $(1.36-2.36)$ & 2.30 & $(1.78-2.99)$ & 3.52 & $(1.35-9.22)$ \\
\hline Shortness of breath & 2.32 & $(1.69-3.19)$ & 1.90 & $(1.42-2.54)$ & 0.65 & $(0.17-2.51)$ \\
\hline Q1 FEV 1 relative slope & 1.00 & & 1.00 & & 1.00 & \\
\hline Q2 $\mathrm{FEV}_{1}$ relative slope & 1.65 & $(1.12-2.45)$ & 2.42 & $(1.68-3.48)$ & 3.05 & $(0.81-11.46)$ \\
\hline Q3 $\mathrm{FEV}_{1}$ relative slope & 2.28 & $(1.55-3.33)$ & 2.97 & $(2.06-4.28)$ & 6.11 & $(1.62-23.09)$ \\
\hline $\mathrm{Q} \mathrm{FEV}_{1}$ relative slope & 3.46 & $(2.44-4.92)$ & 6.01 & $(4.31-8.39)$ & 6.80 & $(1.77-26.20)$ \\
\hline Asthma & 1.24 & $(0.81-1.89)$ & 0.91 & $(0.61-1.37)$ & 4.84 & $(1.28-18.34)$ \\
\hline Chronic bronchitis & 1.80 & $(1.37-2.36)$ & 2.34 & $(1.80-3.03)$ & 3.58 & $(1.37-9.37)$ \\
\hline Asthma & 1.38 & $(0.91-2.09)$ & 0.89 & $(0.59-1.34)$ & 4.78 & $(1.37-16.69)$ \\
\hline Chronic bronchitis & 1.91 & $(1.46-2.51)$ & 2.51 & $(1.94-3.24)$ & 3.37 & $(1.31-8.67)$ \\
\hline Shortness of breath & 2.59 & $(1.89-3.56)$ & 2.18 & $(1.64-2.90)$ & 0.82 & $(0.23-2.97)$ \\
\hline
\end{tabular}

*Models adjusted for age at examination two.

$\dagger$ Asthma, chronic bronchitis, and shortness of breath represented as dichotomous variables in all models.

$\left\lceil\right.$ Models adjusted for baseline age, height-adjusted baseline lung function $\left(\mathrm{FEV}_{1} /\right.$ height $\left.^{2}\right)$, and height. See Table A.1 for quartile values.

$\mathrm{FEV}_{1}$ relative slope, slope $\mathrm{FEV}_{1} /$ baseline $\mathrm{FEV}_{1}$; LNL, Longitudinal Normal Limit. 


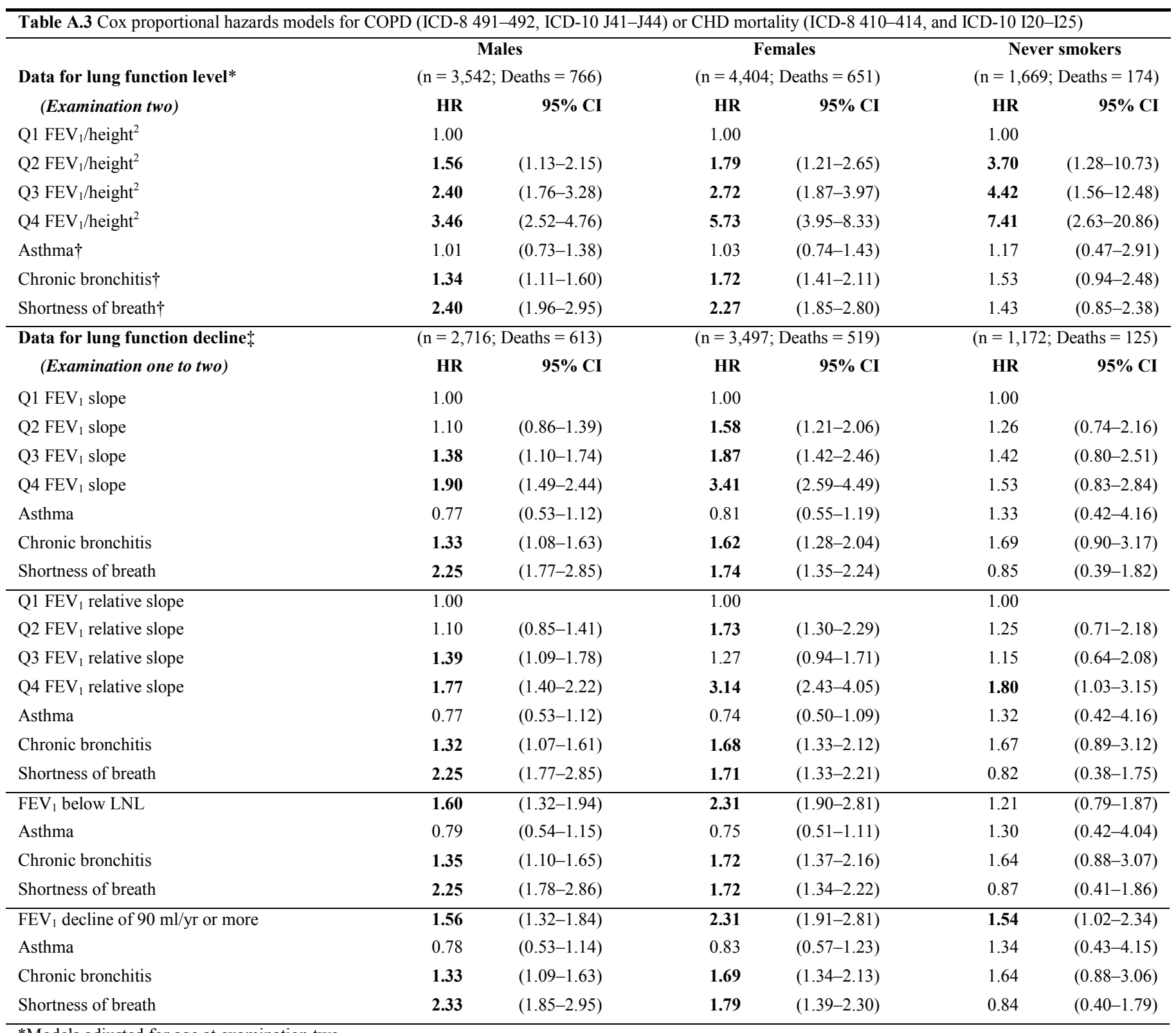

*Models adjusted for age at examination two.

$\uparrow$ Asthma, chronic bronchitis, and shortness of breath represented as dichotomous variables in all models.

$\ddagger$ Models adjusted for baseline age, height-adjusted baseline lung function $\left(\mathrm{FEV}_{1} /\right.$ height $^{2}$ ), and height. See Table A.1 for quartile values.

$\mathrm{FEV}_{1}$ relative slope, slope $\mathrm{FEV}_{1}$ /baseline $\mathrm{FEV}_{1}$; LNL, Longitudinal Normal Limit. 


\begin{tabular}{|c|c|c|c|c|c|c|}
\hline \multirow{3}{*}{$\begin{array}{l}\text { Data for lung function level* } \\
\text { (Examination two) }\end{array}$} & \multicolumn{2}{|c|}{ Males } & \multirow{2}{*}{\multicolumn{2}{|c|}{$\begin{array}{c}\text { Females } \\
(\mathrm{n}=4,404 ; \text { Deaths }=2,352)\end{array}$}} & \multirow{2}{*}{\multicolumn{2}{|c|}{$\begin{array}{c}\text { Never smokers } \\
(\mathrm{n}=1,669 ; \text { Deaths }=766)\end{array}$}} \\
\hline & \multicolumn{2}{|c|}{$(\mathrm{n}=3,542 ;$ Deaths $=2,259)$} & & & & \\
\hline & HR & $95 \%$ CI & \multirow{2}{*}{\multicolumn{2}{|c|}{ HR }} & \multirow{2}{*}{$\begin{array}{r}\text { HR } \\
1.00\end{array}$} & \multirow[t]{2}{*}{$95 \% \mathrm{CI}$} \\
\hline Q1 FEV $1 /$ height $^{2}$ & \multicolumn{2}{|l|}{1.00} & & & & \\
\hline Q2 $\mathrm{FEV}_{1} /$ height $^{2}$ & 1.37 & $(1.17-1.60)$ & 1.44 & $(1.23-1.70)$ & \multicolumn{2}{|l|}{$\begin{array}{l}1.00 \\
1.89\end{array}$} \\
\hline Q3 $\mathrm{FEV}_{1} /$ height $^{2}$ & 1.89 & $(1.61-2.21)$ & 1.88 & $(1.60-2.20)$ & 2.07 & $(1.51-2.85)$ \\
\hline Asthma $\dagger$ & 0.89 & $(0.71-1.11)$ & 1.14 & $(0.92-1.40)$ & 1.16 & $(0.72-1.87)$ \\
\hline Chronic bronchitisł & 1.24 & $(1.11-1.39)$ & 1.38 & $(1.23-1.56)$ & 1.34 & $(1.04-1.73)$ \\
\hline Shortness of breath $\dagger$ & 1.66 & $(1.45-1.90)$ & 1.43 & $(1.26-1.63)$ & 1.27 & $(0.97-1.66)$ \\
\hline Data for lung function decline & \multicolumn{2}{|c|}{$(\mathrm{n}=2,708 ;$ Deaths $=1,796)$} & \multicolumn{2}{|c|}{$(\mathrm{n}=3,486 ;$ Deaths $=1,863)$} & \multicolumn{2}{|c|}{$(\mathrm{n}=1,165 ;$ Deaths $=543)$} \\
\hline (Examination one to two) & HR & $95 \%$ CI & HR & $95 \%$ CI & HR & $95 \% \mathrm{CI}$ \\
\hline Q4 $\mathrm{FEV}_{1}$ slope & 1.76 & $(1.52-2.03)$ & 1.88 & $(1.63-2.16)$ & 1.71 & $(1.28-2.28)$ \\
\hline Asthma & 0.72 & $(0.55-0.94)$ & 1.05 & $(0.82-1.33)$ & 1.25 & $(0.72-2.18)$ \\
\hline Chronic bronchitis & 1.20 & $(1.06-1.36)$ & 1.34 & $(1.17-1.54)$ & 1.40 & $(1.01-1.94)$ \\
\hline Shortness of breath & 1.60 & $(1.36-1.87)$ & 1.27 & $(1.09-1.48)$ & 1.08 & $(0.77-1.52)$ \\
\hline $\mathrm{Q} \mathrm{FEV}_{1}$ relative slope & 1.00 & & 1.00 & & 1.00 & \\
\hline Q2 $\mathrm{FEV}_{1}$ relative slope & 1.15 & $(0.99-1.32)$ & 1.27 & $(1.10-1.45)$ & 1.19 & $(0.91-1.55)$ \\
\hline Q3 $\mathrm{FEV}_{1}$ relative slope & 1.37 & $(1.19-1.57)$ & 1.22 & $(1.06-1.41)$ & 1.21 & $(0.92-1.60)$ \\
\hline $\mathrm{Q} \mathrm{FEV}_{1}$ relative slope & 1.61 & $(1.40-1.84)$ & 1.82 & $(1.59-2.07)$ & 1.61 & $(1.23-2.12)$ \\
\hline Asthma & 0.72 & $(0.55-0.94)$ & 1.01 & $(0.79-1.28)$ & 1.29 & $(0.74-2.25)$ \\
\hline Chronic bronchitis & 1.19 & $(1.05-1.35)$ & 1.37 & $(1.19-1.57)$ & 1.40 & $(1.01-1.95)$ \\
\hline Asthma & 0.73 & $(0.56-0.95)$ & 1.07 & $(0.85-1.37)$ & 1.28 & $(0.74-2.21)$ \\
\hline Chronic bronchitis & 1.21 & $(1.07-1.37)$ & 1.37 & $(1.19-1.57)$ & 1.38 & $(1.00-1.92)$ \\
\hline Shortness of breath & 1.67 & $(1.43-1.96)$ & 1.28 & $(1.09-1.49)$ & 1.08 & $(0.77-1.52)$ \\
\hline
\end{tabular}

*Models adjusted for age at examination two.

$\dagger$ Asthma, chronic bronchitis, and shortness of breath represented as dichotomous variables in all models.

$\$$ Models adjusted for baseline age, height-adjusted baseline lung function $\left(\mathrm{FEV}_{1} /\right.$ height $\left.^{2}\right)$, and height. See Table A.1 for quartile values.

$\mathrm{FEV}_{1}$ relative slope, slope $\mathrm{FEV}_{1} /$ baseline $_{\mathrm{FEV}}$; $\mathrm{LNL}$, Longitudinal Normal Limit. 

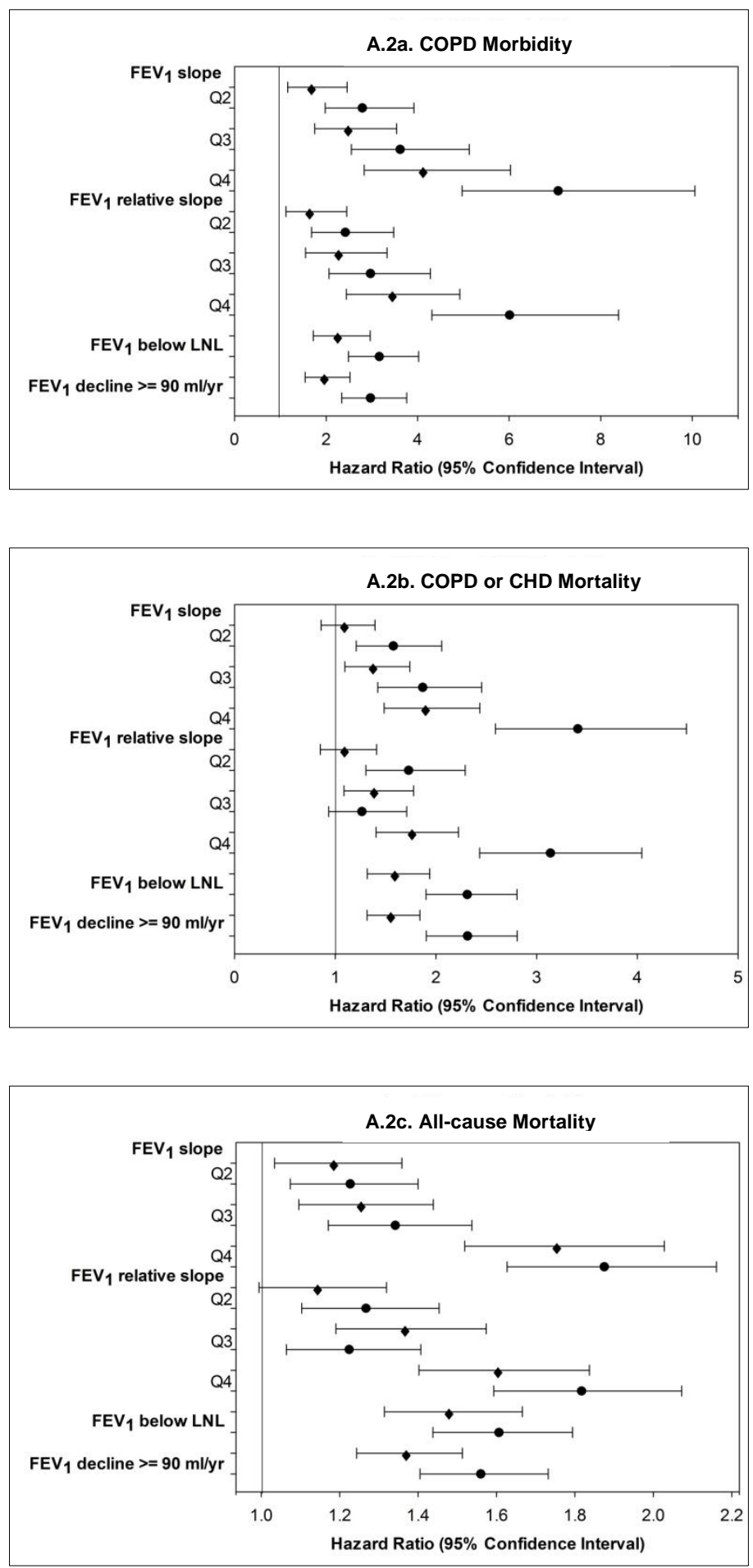

Figure A.2 Cox proportional hazards model results by gender $(\bullet=$ males and $\bullet=$ females) for (a) COPD morbidity, (b) COPD or CHD mortality, and (c) all-cause mortality. Models adjusted for baseline age, height-adjusted baseline lung function $\left(\mathrm{FEV}_{1} / \mathrm{height}^{2}\right)$, height, and respiratory symptoms and asthma as dichotomous variables. Relative slope, $\mathrm{FEV}_{1} /$ baseline $\mathrm{FEV}_{1}$; $\mathrm{LNL}$, Longitudinal Normal Limit. See Table A.1 for quartile values. 

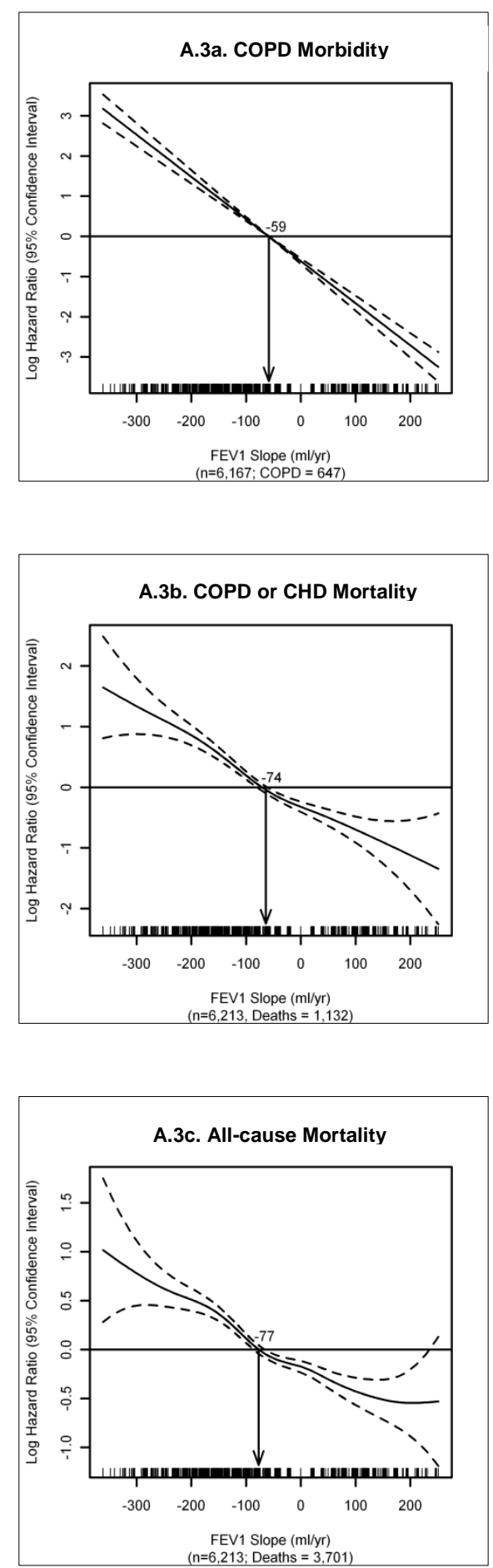

Figure A.3 Cox proportional hazards model using a penalized spline for the $\mathrm{FEV}_{1}$ slope, males and females combined. The spline crossed above zero for the log HR at $-59 \mathrm{ml} / \mathrm{yr}$ for (a) COPD morbidity, at $-74 \mathrm{ml} / \mathrm{yr}$ for (b) COPD or CHD mortality, and at $-77 \mathrm{ml} / \mathrm{yr}$ for (c) all-cause mortality. Models adjusted for baseline age, height-adjusted baseline lung function ( $\mathrm{FEV}_{1} /$ height $\left.^{2}\right)$, and height. Tick marks on the $x$ axis represent the frequency of the various FEV ${ }_{1}$ slope values. 
Appendix B: Sensitivity Analyses for Study 2 


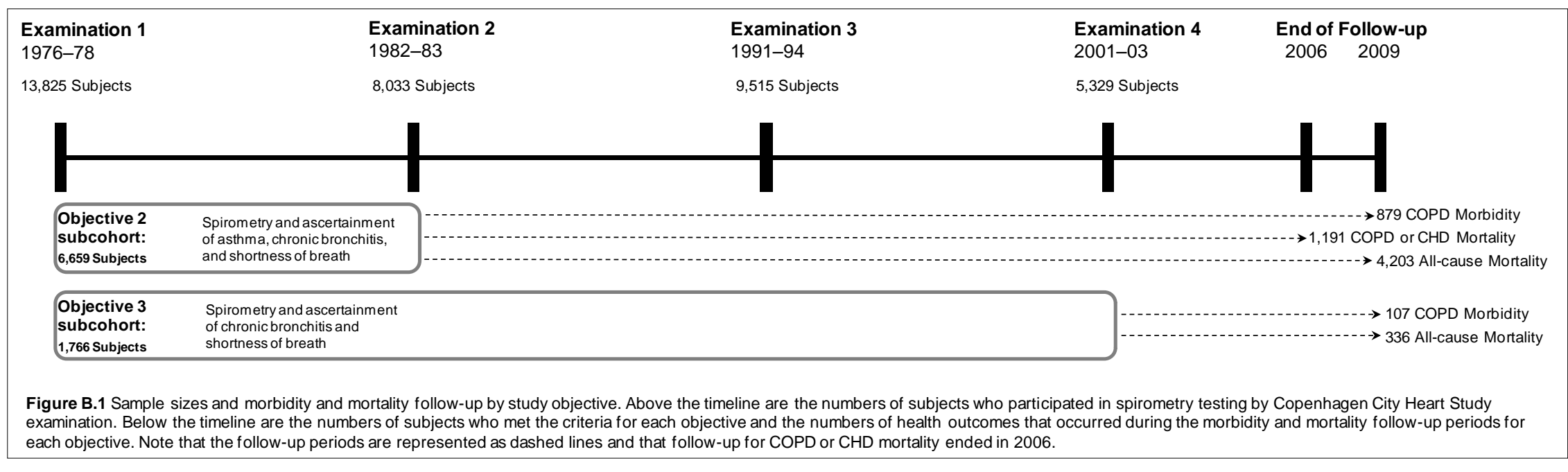




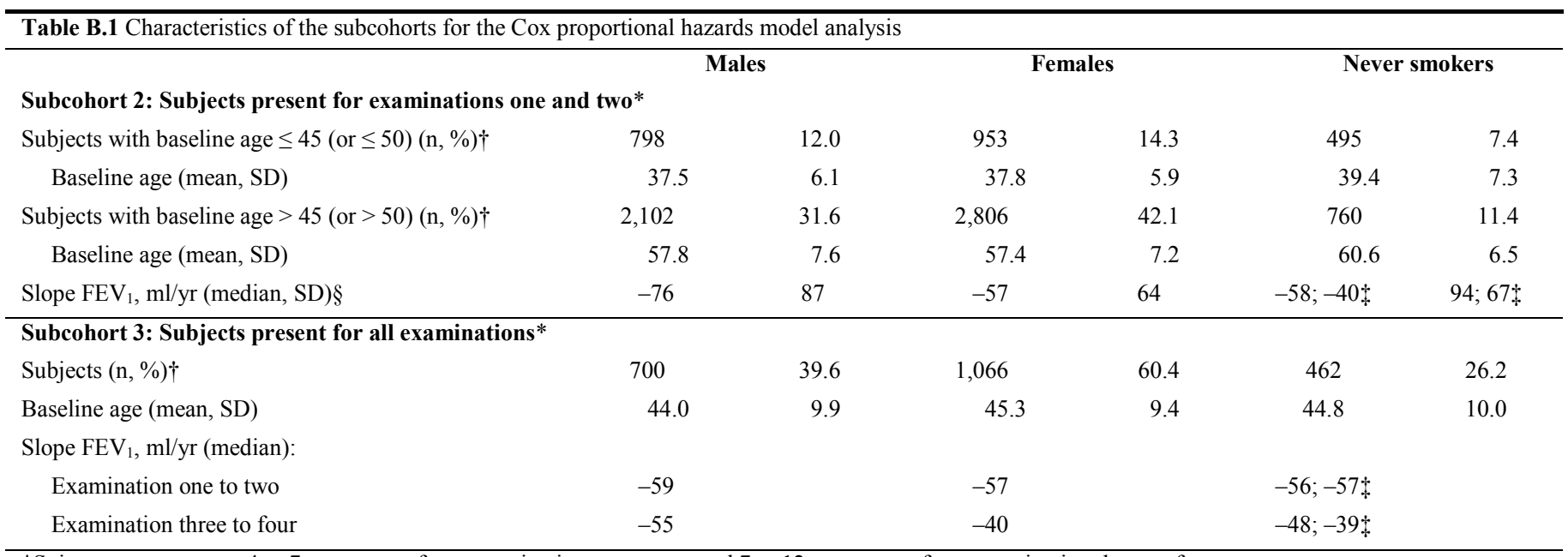

*Spirometry tests were 4 to 7 years apart from examination one to two and 7 to 12 years apart from examination three to four.

$\dagger$ A total of 6,659 subjects were present for examinations one and two and 1,766 for all examinations.

$\vdots$ Males; females.

§Overall median and standard deviation values, not age-stratified.

$\mathrm{FEV}_{1}$, forced expiratory volume in one second; $\mathrm{ml} / \mathrm{yr}$, milliliters/year; SD, standard deviation. 


\begin{tabular}{|c|c|c|c|c|c|c|c|c|c|c|c|c|}
\hline & \multicolumn{4}{|c|}{ Males } & \multicolumn{4}{|c|}{ Females } & \multicolumn{4}{|c|}{ Never Smokers } \\
\hline & \multicolumn{2}{|c|}{$\begin{array}{c}\text { Baseline Age } \\
\leq 45 \text { Years }\end{array}$} & \multicolumn{2}{|c|}{$\begin{array}{c}\text { Baseline Age } \\
>45 \text { Years }\end{array}$} & \multicolumn{2}{|c|}{$\begin{array}{c}\text { Baseline Age } \\
\leq 45 \text { Years }\end{array}$} & \multicolumn{2}{|c|}{$\begin{array}{c}\text { Baseline Age } \\
>45 \text { Years }\end{array}$} & \multicolumn{2}{|c|}{$\begin{array}{l}\text { Baseline Age } \\
\leq 50 \text { Years }\end{array}$} & \multicolumn{2}{|c|}{$\begin{array}{c}\text { Baseline Age } \\
>50 \text { Years }\end{array}$} \\
\hline \multirow[t]{2}{*}{ COPD Morbidity } & \multicolumn{2}{|c|}{$(\mathrm{n}=796 ; \mathrm{COPD}=82)$} & \multicolumn{2}{|c|}{$(\mathrm{n}=2,705 ; \mathrm{COPD}=295)$} & \multicolumn{2}{|c|}{$(\mathrm{n}=950 ; \mathrm{COPD}=92)$} & \multicolumn{2}{|c|}{$(\mathrm{n}=2,788 ; \mathrm{COPD}=410)$} & \multicolumn{2}{|c|}{$(\mathrm{n}=373 ; \mathrm{COPD}=4)$} & \multicolumn{2}{|c|}{$(\mathrm{n}=879 ; \mathrm{COPD}=47)$} \\
\hline & HR & $95 \%$ CI & HR & $95 \%$ CI & HR & $95 \% \mathrm{CI}$ & HR & $95 \%$ CI & HR & $95 \%$ CI & HR & $95 \%$ CI \\
\hline Q1 FEV 1 slope & 1.00 & & 1.00 & & 1.00 & & 1.00 & & 1.00 & & 1.00 & \\
\hline Q2 $\mathrm{FEV}_{1}$ slope & 1.16 & $(0.63-2.16)$ & 2.05 & $(1.39-3.02)$ & 1.97 & $(1.05-3.68)$ & 2.23 & $(1.64-3.04)$ & 0.43 & $(0.05-3.65)$ & 1.18 & $(0.44-3.13)$ \\
\hline Q3 FEV 1 slope & 2.14 & $(1.18-3.88)$ & 2.72 & $(1.88-3.93)$ & 2.51 & $(1.29-4.87)$ & 2.90 & $(2.11-3.97)$ & 1.33 & $(0.20-8.79)$ & 2.22 & $(0.83-5.94)$ \\
\hline Q4 FEV 1 slope & 1.67 & $(0.82-3.44)$ & 5.21 & $(3.53-7.68)$ & 5.53 & $(2.92-10.47)$ & 4.89 & $(3.53-6.78)$ & 0.00 & $(0.00-0.00)$ & 2.52 & $(0.88-7.18)$ \\
\hline Asthma & 1.03 & $(0.24-4.48)$ & 1.50 & $(0.98-2.28)$ & 1.14 & $(0.47-2.78)$ & 1.05 & $(0.70-1.56)$ & 29.25 & $(4.48-191.20)$ & 2.41 & $(0.60-9.75)$ \\
\hline Chronic bronchitis & 1.75 & $(1.00-3.05)$ & 1.82 & $(1.38-2.40)$ & 4.15 & $(2.52-6.82)$ & 2.05 & $(1.60-2.63)$ & 21.97 & $(3.01-160.34)$ & 2.25 & $(0.82-6.18)$ \\
\hline Shortness of breath & 1.46 & $(0.61-3.50)$ & 1.89 & $(1.37-2.62)$ & 1.17 & $(0.63-2.19)$ & 2.18 & $(1.66-2.86)$ & 0.74 & $(0.06-9.69)$ & 1.64 & $(0.59-4.52)$ \\
\hline \multirow[t]{2}{*}{ COPD or CHD Mortality } & \multicolumn{2}{|c|}{$(\mathrm{n}=798 ;$ Deaths $=50)$} & \multicolumn{2}{|c|}{$(\mathrm{n}=2,102 ;$ Deaths $=592)$} & \multicolumn{2}{|c|}{$(\mathrm{n}=953 ;$ Deaths $=29)$} & \multicolumn{2}{|c|}{$(\mathrm{n}=2,806 ;$ Deaths $=520)$} & \multicolumn{2}{|c|}{$(\mathrm{n}=373 ;$ Deaths $=1)$} & \multicolumn{2}{|c|}{$(\mathrm{n}=882 ;$ Deaths $=127)$} \\
\hline & HR & $95 \% \mathrm{CI}$ & HR & $95 \% \mathrm{CI}$ & HR & $95 \%$ CI & HR & $95 \%$ CI & HR & $95 \% \mathrm{CI}$ & HR & $95 \% \mathrm{CI}$ \\
\hline Q1 FEV 1 slope & 1.00 & & 1.00 & & 1.00 & & 1.00 & & 1.00 & & 1.00 & \\
\hline Q2 $\mathrm{FEV}_{1}$ slope & 1.27 & $(0.57-2.83)$ & 1.14 & $(0.89-1.46)$ & 1.97 & $(0.66-5.84)$ & 1.64 & $(1.25-2.14)$ & 2.37 & $(0.23-24.25)$ & 1.18 & $(0.68-2.03)$ \\
\hline Q3 $\mathrm{FEV}_{1}$ slope & 1.97 & $(0.89-4.37)$ & 1.41 & $(1.11-1.79)$ & 1.88 & $(0.57-6.25)$ & 1.93 & $(1.47-2.53)$ & 4.46 & $(0.42-47.14)$ & 1.31 & $(0.74-2.31)$ \\
\hline Q4 $\mathrm{FEV}_{1}$ slope & 2.25 & $(0.95-5.35)$ & 1.93 & $(1.50-2.49)$ & 5.64 & $(1.88-16.85)$ & 3.17 & $(2.39-4.19)$ & 1.42 & $(0.08-26.81)$ & 1.41 & $(0.76-2.63)$ \\
\hline Asthma & 1.55 & $(0.46-5.22)$ & 0.79 & $(0.54-1.18)$ & 1.25 & $(0.30-5.15)$ & 0.73 & $(0.49-1.09)$ & 2.94 & $(0.26-32.66)$ & 0.93 & $(0.26-3.36)$ \\
\hline Chronic bronchitis & 1.06 & $(0.51-2.23)$ & 1.32 & $(1.07-1.62)$ & 1.80 & $(0.69-4.68)$ & 1.50 & $(1.18-1.90)$ & 2.16 & $(0.21-21.77)$ & 1.54 & $(0.81-2.95)$ \\
\hline Shortness of breath & 4.25 & $(1.92-9.41)$ & 2.11 & $(1.66-2.69)$ & 1.40 & $(0.48-4.11)$ & 1.91 & $(1.49-2.45)$ & 2.55 & $(0.28-23.41)$ & 1.05 & $(0.52-2.14)$ \\
\hline \multirow[t]{2}{*}{ All-cause Mortality } & \multicolumn{2}{|c|}{$(\mathrm{n}=798 ;$ Deaths $=251)$} & \multicolumn{2}{|c|}{$(\mathrm{n}=2,102 ;$ Deaths $=1,752)$} & \multicolumn{2}{|c|}{$(n=953 ;$ Deaths $=204)$} & \multicolumn{2}{|c|}{$(\mathrm{n}=2,806 ;$ Deaths $=1,996)$} & $(n=3$ & 3; Deaths=47) & $(\mathrm{n}=88$ & Deaths $=580$ ) \\
\hline & HR & $95 \%$ CI & HR & $95 \% \mathrm{CI}$ & HR & $95 \% \mathrm{CI}$ & HR & $95 \% \mathrm{CI}$ & HR & $95 \% \mathrm{CI}$ & HR & $95 \% \mathrm{CI}$ \\
\hline Q1 FEV 1 slope & 1.00 & & 1.00 & & 1.00 & & 1.00 & & 1.00 & & 1.00 & \\
\hline Q2 $\mathrm{FEV}_{1}$ slope & 1.00 & $(0.70-1.44)$ & 1.23 & $(1.07-1.41)$ & 1.40 & $(0.95-2.07)$ & 1.32 & $(1.16-1.51)$ & 0.69 & $(0.35-1.37)$ & 1.22 & $(0.94-1.58)$ \\
\hline Q3 FEV 1 slope & 1.64 & $(1.15-2.33)$ & 1.22 & $(1.06-1.40)$ & 1.24 & $(0.81-1.88)$ & 1.42 & $(1.24-1.62)$ & 1.22 & $(0.63-2.38)$ & 1.26 & $(0.95-1.65)$ \\
\hline Q4 $\mathrm{FEV}_{1}$ slope & 2.13 & $(1.47-3.08)$ & 1.67 & $(1.44-1.94)$ & 1.89 & $(1.23-2.89)$ & 1.92 & $(1.66-2.20)$ & 1.20 & $(0.59-2.45)$ & 1.51 & $(1.13-2.02)$ \\
\hline Asthma & 0.55 & $(0.20-1.50)$ & 0.80 & $(0.62-1.04)$ & 0.92 & $(0.44-1.91)$ & 1.03 & $(0.81-1.32)$ & 1.16 & $(0.27-5.00)$ & 1.11 & $(0.62-1.99)$ \\
\hline Chronic bronchitis & 1.28 & $(0.91-1.80)$ & 1.19 & $(1.05-1.35)$ & 1.11 & $(0.70-1.75)$ & 1.36 & $(1.19-1.56)$ & 1.32 & $(0.40-4.36)$ & 1.48 & $(1.08-2.02)$ \\
\hline Shortness of breath & 1.87 & $(1.16-3.00)$ & 1.57 & $(1.34-1.85)$ & 1.32 & $(0.84-2.08)$ & 1.28 & $(1.11-1.49)$ & 1.66 & $(0.64-4.29)$ & 1.06 & $(0.76-1.48)$ \\
\hline
\end{tabular}

*Adjusted for baseline age, height-adjusted baseline lung function $\left(\mathrm{FEV}_{1} /\right.$ height $\left.^{3}\right)$, and height.

$\dagger$ Asthma and respiratory symptoms ascertained at examination two and represented as dichotomous variables in all models. 


\begin{tabular}{|c|c|c|c|c|c|c|}
\hline \multirow[t]{2}{*}{ COPD Morbidity } & \multicolumn{2}{|c|}{$\begin{array}{c}\text { Males } \\
(\mathrm{n}=675 ; \text { COPD }=45)\end{array}$} & \multicolumn{2}{|c|}{$\begin{array}{c}\text { Females } \\
(\mathrm{n}=1,022 ; \text { COPD }=62)\end{array}$} & \multicolumn{2}{|c|}{$\begin{array}{c}\text { Never smokers } \\
(\mathrm{n}=453 ; \mathrm{COPD}=8) \S\end{array}$} \\
\hline & HR & $95 \% \mathrm{CI}$ & HR & $95 \% \mathrm{CI}$ & & \\
\hline \multicolumn{7}{|l|}{ Slope FEV 1} \\
\hline 1) Normal decline over both periods & 1.00 & & 1.00 & & & \\
\hline 2) Excessive decline over period one only & 3.26 & $(0.86-12.33)$ & 3.13 & $(1.17-8.35)$ & & \\
\hline 4) Excessive decline over both periods & 10.49 & $(3.00-36.70)$ & 4.06 & $(1.53-10.74)$ & & \\
\hline \multicolumn{7}{|l|}{ Chronic bronchitis } \\
\hline 1) Absent in both periods & 1.00 & & 1.00 & & & \\
\hline 2) Present in period one only & 0.00 & $(0.00-0.00)$ & 2.61 & $(0.74-9.15)$ & & \\
\hline 3) Present in period two only & 1.86 & $(0.91-3.83)$ & 1.74 & $(0.91-3.32)$ & & \\
\hline 2) Present in period one only! & 0.00 & $(0.00-0.00)$ & 2.07 & $(0.47-9.14)$ & & \\
\hline 3) Present in period two only & 3.75 & $(1.86-7.55)$ & 6.35 & $(3.50-11.53)$ & & \\
\hline 4) Present in both periods & 5.91 & $(1.38-25.39)$ & 4.34 & $(1.72-10.93)$ & & \\
\hline \multirow[t]{2}{*}{ All-cause Mortality } & \multicolumn{2}{|c|}{$(\mathrm{n}=700 ;$ Deaths $=152)$} & \multicolumn{2}{|c|}{$(\mathrm{n}=1,066 ;$ Deaths $=184)$} & \multicolumn{2}{|c|}{$(\mathrm{n}=462 ;$ Deaths $=59)$} \\
\hline & HR & $95 \% \mathrm{CI}$ & HR & $95 \% \mathrm{CI}$ & HR & $95 \% \mathrm{CI}$ \\
\hline \multicolumn{7}{|l|}{ Slope FEV $F_{1}$} \\
\hline 1) Normal decline over both periods & 1.00 & & 1.00 & & 1.00 & \\
\hline 2) Excessive decline over period one only & 1.24 & $(0.73-2.11)$ & 1.23 & $(0.78-1.96)$ & 2.04 & $(0.87-4.77)$ \\
\hline 3) Excessive decline over period two only & 1.91 & $(1.16-3.12)$ & 1.43 & $(0.91-2.23)$ & 1.41 & $(0.58-3.42)$ \\
\hline 4) Excessive decline over both periods & 2.17 & $(1.30-3.62)$ & 1.64 & $(1.03-2.62)$ & 1.62 & $(0.66-3.94)$ \\
\hline 1) Absent in both periods & 1.00 & & 1.00 & & 1.00 & \\
\hline 2) Present in period one only & 0.68 & $(0.09-4.88)$ & 1.12 & $(0.35-3.56)$ & 3.40 & $(0.43-26.76)$ \\
\hline 3) Present in period two only & 1.97 & $(1.21-3.21)$ & 1.71 & $(1.11-2.63)$ & 1.19 & $(0.42-3.40)$ \\
\hline 4) Present in both periods & 1.91 & $(0.66-5.51)$ & 1.64 & $(0.78-3.47)$ & 0.54 & $(0.07-4.44)$ \\
\hline
\end{tabular}

*Adjusted for baseline age, height-adjusted baseline lung function $\left(\mathrm{FEV}_{1} /\right.$ height $\left.^{3}\right)$, and height.

$\uparrow$ Respiratory symptoms ascertained at examination two and represented as dichotomous variables in all models.

$\$$ Note that no COPD morbidity events occurred for males in pattern 2 for shortness of breath.

$\S$ Sensitivity analysis was not possible due to too few cases overall after excluding the adjusted $1981 \mathrm{FEV}_{1}$ values.

$\mathrm{FEV}_{1}$, forced expiratory volume in one second; $\mathrm{ml} / \mathrm{yr}$, milliliters/year; SD, standard deviation. 
Appendix C: Sensitivity Analyses for Study 3 


Examination 1
$1976-78$




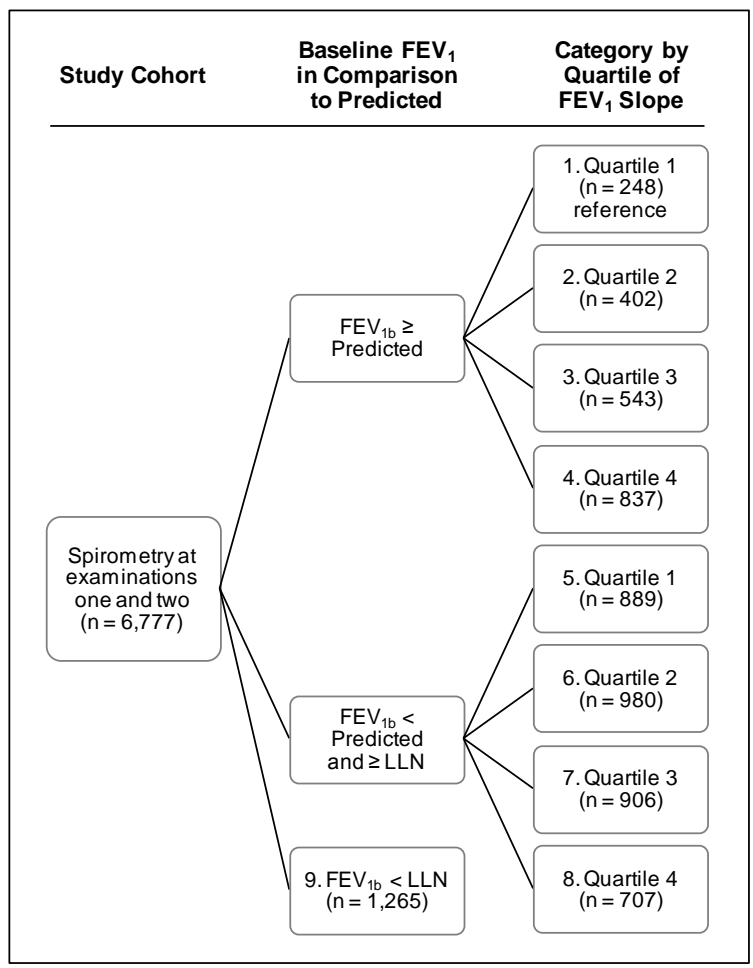

Figure C.2 Nine lung function categories based on baseline $\mathrm{FEV}_{1}$ values in comparison to predicted values and quartiles of $F E V_{1}$ slope. European reference equations were used to calculate the predicted $\mathrm{FEV}_{1}$ values. ${ }^{111} \mathrm{FEV}_{1 \mathrm{~b}}$, baseline forced expiratory volume in one second; LLN, lower limit of normal. 


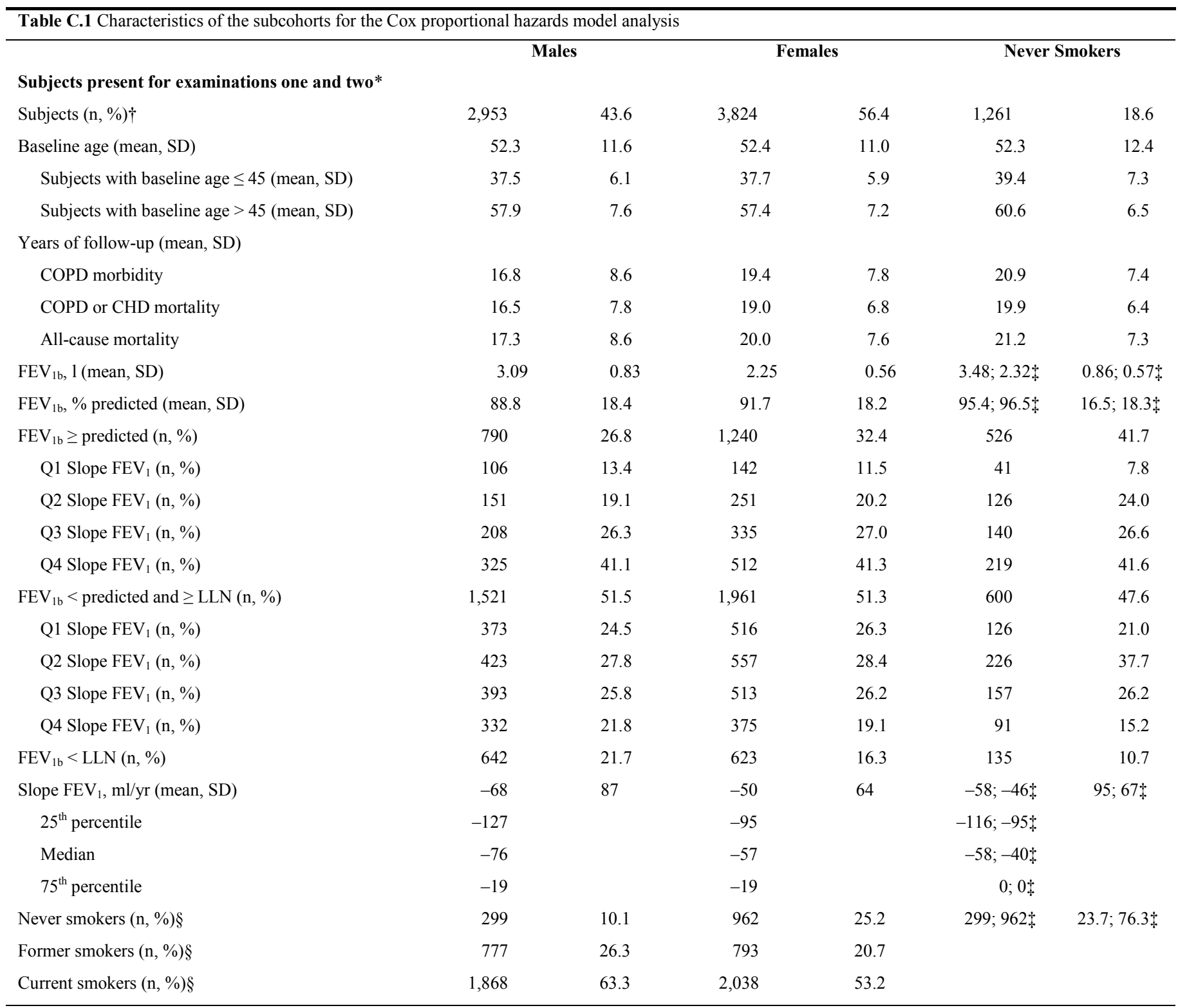

*Spirometry tests were 4 to 7 years apart from examination one to two with a mean follow-up of 5 years.

$\dagger$ A total of 6,777 subjects were present for examinations one and two.

\$Males; females.

$\S$ Ascertained at examination two.

$\mathrm{FEV}_{1 \mathrm{~b}}$, baseline forced expiratory volume in one second; 1, liters; LLN, lower limit of normal; ml/yr, milliliters/year; SD, standard deviation. 

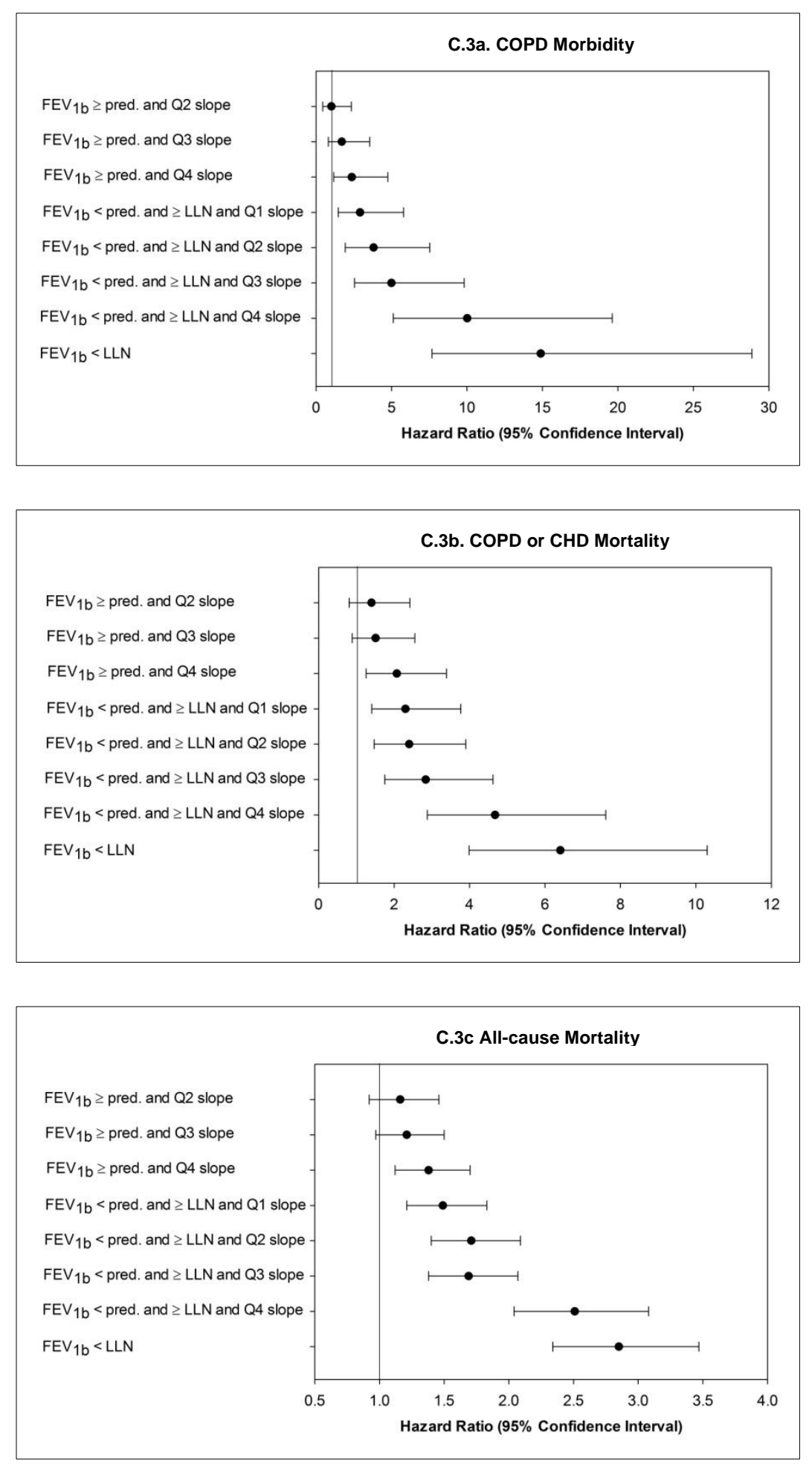

Figure C.3 Cox proportional hazards model results for overall (a) morbidity, (b) COPD or CHD mortality, and (c) all-cause mortality risks by lung function category. Models adjusted for baseline age and height. $\mathrm{FEV}_{1 \mathrm{~b}}$, baseline forced expiratory volume in one second; LLN, lower limit of normal; pred., predicted value. 


\begin{tabular}{|c|c|c|c|}
\hline & HR & $95 \% \mathrm{CI}$ & PAR (\%) \\
\hline COPD Morbidity & \multicolumn{2}{|c|}{$(\mathrm{n}=6,721 ; \mathrm{COPD}=928)$} & \\
\hline Categories $1-8$ & 1.00 & & \\
\hline Category 9 & 4.11 & $(3.59-4.71)$ & 36.2 \\
\hline Categories 1-7 & 1.00 & & \\
\hline Categories 8 and 9 & 4.45 & $(3.90-5.08)$ & 49.7 \\
\hline COPD or CHD Mortality & $(\mathrm{n}=6,777$ & aths $=1,242)$ & \\
\hline Categories $1-8$ & 1.00 & & \\
\hline Category 9 & 2.64 & $(2.33-2.99)$ & 23.4 \\
\hline Categories 1-7 & 1.00 & & \\
\hline Categories 8 and 9 & 2.67 & $(2.38-3.00)$ & 32.7 \\
\hline All-cause Mortality & $(\mathrm{n}=6,777$ & aths $=4,305)$ & \\
\hline Categories $1-8$ & 1.00 & & \\
\hline Category 9 & 1.80 & $(1.68-1.94)$ & 13.0 \\
\hline Categories 1-7 & 1.00 & & \\
\hline Categories 8 and 9 & 1.86 & $(1.74-1.98)$ & 20.0 \\
\hline
\end{tabular}

*Cox models adjusted for baseline age and height. 


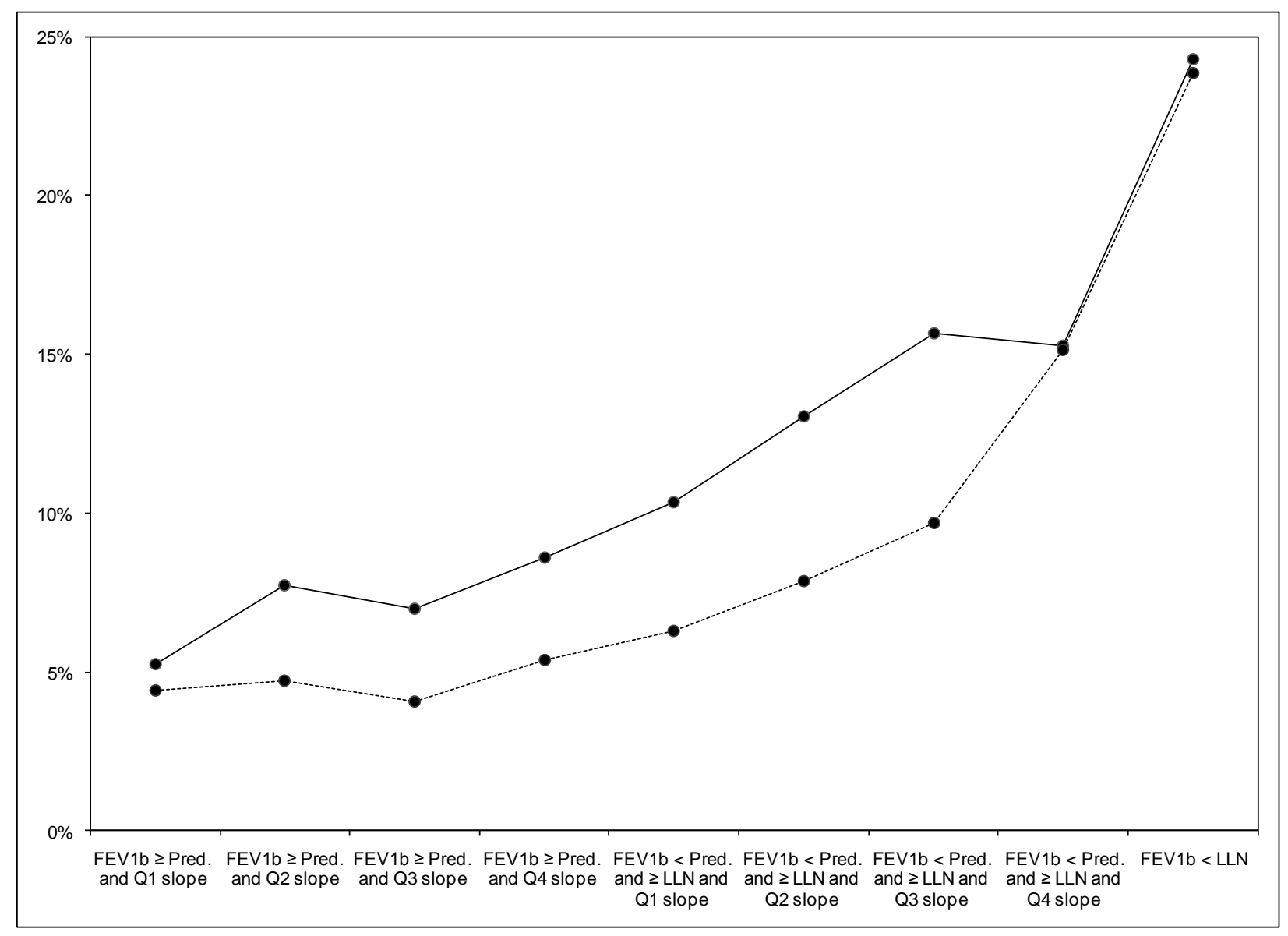

Figure C.4 Overall prevalence rates of self-reported respiratory symptoms (chronic bronchitis = solid line; shortness of breath = dashed line) at examination two by lung function category. The Spearman's rho rank correlations were $-0.97(p<0.0001)$ between the lung function categories and chronic bronchitis and $-0.95(p<0.0001)$ between the lung function categories and shortness of breath. $\mathrm{FEV}_{1 \mathrm{~b}}$, baseline forced expiratory volume in one second; LLN, lower limit of normal; pred., predicted value. 


\begin{tabular}{|c|c|c|c|c|c|c|}
\hline \multirow[b]{3}{*}{ COPD Morbidity } & \multicolumn{2}{|c|}{ Males } & \multicolumn{2}{|c|}{ Females } & \multicolumn{2}{|c|}{ Never Smokers } \\
\hline & HR & $95 \% \mathrm{CI}$ & HR & $95 \%$ CI & HR & $95 \% \mathrm{CI}$ \\
\hline & \multicolumn{2}{|c|}{$(\mathrm{n}=2,919 ; \mathrm{COPD}=401)$} & \multicolumn{2}{|c|}{$(\mathrm{n}=3,802 ; \mathrm{COPD}=527)$} & \multicolumn{2}{|c|}{$(\mathrm{n}=1,258 ; \mathrm{COPD}=52)$} \\
\hline $\mathrm{FEV}_{1 \mathrm{~b}} \geq$ pred. and Q1 slope & \multicolumn{2}{|l|}{1.00} & \multicolumn{2}{|l|}{1.00} & \multicolumn{2}{|l|}{1.00} \\
\hline $\mathrm{FEV}_{1 \mathrm{~b}} \geq$ pred. and Q2 slope & 1.00 & $(0.32-3.16)$ & 1.08 & $(0.32-3.67)$ & 0.10 & $(0.01-1.01)$ \\
\hline $\mathrm{FEV}_{1 \mathrm{~b}} \geq$ pred. and Q3 slope & 1.62 & $(0.60-4.38)$ & 1.75 & $(0.58-5.26)$ & 0.11 & $(0.01-1.01)$ \\
\hline $\mathrm{FEV}_{1 \mathrm{~b}} \geq$ pred. and Q4 slope & 1.52 & $(0.57-4.02)$ & 3.49 & $(1.25-9.74)$ & 0.33 & $(0.08-1.36)$ \\
\hline $\mathrm{FEV}_{1 \mathrm{~b}}<$ pred. and $\geq$ LLN and Q1 slope & 1.35 & $(0.51-3.57)$ & 4.95 & $(1.79-13.67)$ & 0.60 & $(0.14-2.51)$ \\
\hline $\mathrm{FEV}_{1 \mathrm{~b}}<$ pred. and $\geq$ LLN and Q2 slope & 1.93 & $(0.75-4.93)$ & 6.25 & $(2.28-17.09)$ & 0.72 & $(0.20-2.62)$ \\
\hline $\mathrm{FEV}_{1 \mathrm{~b}}<$ pred. and $\geq$ LLN and Q3 slope & 3.70 & $(1.48-9.25)$ & 6.61 & $(2.41-18.12)$ & 1.21 & $(0.34-4.32)$ \\
\hline $\mathrm{FEV}_{1 \mathrm{~b}}<$ pred. and $\geq$ LLN and Q4 slope & 6.90 & $(2.77-17.15)$ & 14.24 & $(5.22-38.85)$ & 1.42 & $(0.37-5.52)$ \\
\hline $\mathrm{FEV}_{1 \mathrm{~b}}<\mathrm{LLN}$ & 10.36 & $(4.25-25.26)$ & 20.81 & $(7.71-56.19)$ & 1.41 & $(0.38-5.23)$ \\
\hline COPD or CHD Mortality & \multicolumn{2}{|c|}{$(\mathrm{n}=2,953 ;$ Deaths $=669)$} & \multicolumn{2}{|c|}{$(n=3,824 ;$ Deaths $=573)$} & \multicolumn{2}{|c|}{$(\mathrm{n}=1,261 ;$ Deaths $=129) \dagger$} \\
\hline $\mathrm{FEV}_{\mathrm{lb}} \geq$ pred. and Q1 slope & \multicolumn{2}{|l|}{1.00} & \multicolumn{2}{|l|}{1.00} & & \\
\hline $\mathrm{FEV}_{1 \mathrm{~b}} \geq$ pred. and Q2 slope & 0.95 & $(0.46-1.94)$ & 2.19 & $(0.89-5.37)$ & & \\
\hline $\mathrm{FEV}_{1 \mathrm{~b}} \geq$ pred. and Q3 slope & 0.98 & $(0.50-1.90)$ & 2.36 & $(0.98-5.66)$ & & \\
\hline $\mathrm{FEV}_{1 \mathrm{~b}} \geq$ pred. and Q4 slope & 1.27 & $(0.68-2.36)$ & 3.75 & $(1.62-8.66)$ & & \\
\hline $\mathrm{FEV}_{1 \mathrm{~b}}<$ pred. and $\geq$ LLN and Q1 slope & 1.32 & $(0.71-2.44)$ & 4.06 & $(1.75-9.39)$ & & \\
\hline $\mathrm{FEV}_{1 \mathrm{~b}}<$ pred. and $\geq$ LLN and Q2 slope & 1.34 & $(0.73-2.47)$ & 4.48 & $(1.95-10.28)$ & & \\
\hline $\mathrm{FEV}_{1 \mathrm{~b}}<$ pred. and $\geq$ LLN and Q3 slope & 1.68 & $(0.92-3.07)$ & 5.08 & $(2.21-11.69)$ & & \\
\hline $\mathrm{FEV}_{1 \mathrm{~b}}<$ pred. and $\geq$ LLN and Q4 slope & 2.81 & $(1.54-5.12)$ & 8.77 & $(3.82-20.18)$ & & \\
\hline $\mathrm{FEV}_{1 \mathrm{~b}}<\mathrm{LLN}$ & 3.24 & $(1.81-5.81)$ & 14.80 & $(6.53-33.56)$ & & \\
\hline All-cause Mortality & \multicolumn{2}{|c|}{$(\mathrm{n}=2,953 ;$ Deaths $=2,054)$} & \multicolumn{2}{|c|}{$(\mathrm{n}=3,824 ;$ Deaths $=2,251)$} & \multicolumn{2}{|c|}{$(\mathrm{n}=1,261 ;$ Deaths $=632)$} \\
\hline $\mathrm{FEV}_{1 \mathrm{~b}} \geq$ pred. and Q1 slope & \multicolumn{2}{|l|}{1.00} & \multicolumn{2}{|l|}{1.00} & \multicolumn{2}{|l|}{1.00} \\
\hline $\mathrm{FEV}_{1 \mathrm{~b}} \geq$ pred. and Q2 slope & 0.98 & $(0.68-1.41)$ & 1.27 & $(0.94-1.72)$ & 1.39 & $(0.78-2.45)$ \\
\hline $\mathrm{FEV}_{1 \mathrm{~b}} \geq$ pred. and Q3 slope & 0.97 & $(0.69-1.36)$ & 1.36 & $(1.02-1.82)$ & 1.57 & $(0.89-2.75)$ \\
\hline $\mathrm{FEV}_{1 \mathrm{~b}} \geq$ pred. and $\mathrm{Q} 4$ slope & 1.21 & $(0.88-1.66)$ & 1.57 & $(1.19-2.07)$ & 1.49 & $(0.87-2.56)$ \\
\hline $\mathrm{FEV}_{1 \mathrm{~b}}<$ pred. and $\geq \mathrm{LLN}$ and Q1 slope & 1.29 & $(0.95-1.76)$ & 1.63 & $(1.24-2.16)$ & 1.51 & $(0.85-2.66)$ \\
\hline $\mathrm{FEV}_{1 \mathrm{~b}}<$ pred. and $\geq \mathrm{LLN}$ and Q2 slope & 1.48 & $(1.09-2.01)$ & 1.89 & $(1.44-2.48)$ & 1.74 & $(1.01-2.99)$ \\
\hline $\mathrm{FEV}_{1 \mathrm{~b}}<$ pred. and $\geq \mathrm{LLN}$ and Q3 slope & 1.53 & $(1.12-2.07)$ & 1.84 & $(1.40-2.42)$ & 1.60 & $(0.92-2.78)$ \\
\hline $\mathrm{FEV}_{1 \mathrm{~b}}<$ pred. and $\geq \mathrm{LLN}$ and $\mathrm{Q} 4$ slope & 2.31 & $(1.70-3.14)$ & 2.72 & $(2.06-3.59)$ & 2.50 & $(1.42-4.41)$ \\
\hline $\mathrm{FEV}_{1 \mathrm{~b}}<\mathrm{LLN}$ & 2.46 & $(1.83-3.31)$ & 3.28 & $(2.51-4.30)$ & 2.28 & $(1.31-3.99)$ \\
\hline
\end{tabular}

*Adjusted for baseline age and height.

$\left\lceil\right.$ Sensitivity analysis was not possible due to a lack of cases in the reference category after excluding the adjusted $1981 \mathrm{FEV}_{1}$ values.

$\mathrm{FEV}_{1 \mathrm{~b}}$, baseline forced expiratory volume in one second; LLN, lower limit of normal; pred., predicted value. 


\begin{tabular}{|c|c|c|c|c|}
\hline & \multicolumn{2}{|c|}{ Baseline Age $\leq 45$} & \multicolumn{2}{|c|}{ Baseline Age $>45$} \\
\hline & HR & $95 \% \mathrm{CI}$ & HR & $95 \% \mathrm{CI}$ \\
\hline COPD Morbidity & \multicolumn{2}{|c|}{$(\mathrm{n}=1,768 ; \mathrm{COPD}=178)$} & \multicolumn{2}{|c|}{$(\mathrm{n}=4,953 ; \mathrm{COPD}=750)$} \\
\hline $\mathrm{FEV}_{1 \mathrm{~b}} \geq$ pred. and Q1 slope & 1.00 & & 1.00 & \\
\hline $\mathrm{FEV}_{1 \mathrm{~b}} \geq$ pred. and Q2 slope & 0.96 & $(0.21-4.28)$ & 1.08 & $(0.39-2.97)$ \\
\hline $\mathrm{FEV}_{1 \mathrm{~b}} \geq$ pred. and Q3 slope & 1.92 & $(0.52-7.09)$ & 1.69 & $(0.69-4.14)$ \\
\hline $\mathrm{FEV}_{1 \mathrm{~b}} \geq$ pred. and $\mathrm{Q} 4$ slope & 1.64 & $(0.46-5.87)$ & 2.69 & $(1.15-6.28)$ \\
\hline $\mathrm{FEV}_{1 \mathrm{~b}}<$ pred. and $\geq$ LLN and Q1 slope & 2.54 & $(0.76-8.49)$ & 3.19 & $(1.37-7.40)$ \\
\hline $\mathrm{FEV}_{1 \mathrm{~b}}<$ pred. and $\geq$ LLN and Q2 slope & 2.66 & $(0.79-8.92)$ & 4.38 & $(1.92-10.03)$ \\
\hline $\mathrm{FEV}_{1 \mathrm{~b}}<$ pred. and $\geq$ LLN and Q3 slope & 3.23 & $(0.97-10.82)$ & 5.75 & $(2.52-13.10)$ \\
\hline $\mathrm{FEV}_{1 \mathrm{~b}}<$ pred. and $\geq \mathrm{LLN}$ and Q4 slope & 6.39 & $(1.94-21.05)$ & 11.30 & $(4.97-25.67)$ \\
\hline $\mathrm{FEV}_{1 \mathrm{~b}}<\mathrm{LLN}$ & 7.83 & $(2.45-24.99)$ & 17.70 & $(7.88-39.79)$ \\
\hline COPD or CHD Mortality & \multicolumn{2}{|c|}{$(\mathrm{n}=1,773 ;$ Deaths $=81)$} & \multicolumn{2}{|c|}{$(\mathrm{n}=5,004 ;$ Deaths $=1,161)$} \\
\hline $\mathrm{FEV}_{1 \mathrm{~b}} \geq$ pred. and Q1 slope & 1.00 & & \multicolumn{2}{|c|}{1.00} \\
\hline $\mathrm{FEV}_{1 \mathrm{~b}} \geq$ pred. and Q2 slope & 0.24 & $(0.03-2.31)$ & 1.62 & $(0.90-2.92)$ \\
\hline $\mathrm{FEV}_{1 \mathrm{~b}} \geq$ pred. and Q3 slope & 1.04 & $(0.25-4.35)$ & 1.62 & $(0.92-2.86)$ \\
\hline $\mathrm{FEV}_{1 \mathrm{~b}} \geq$ pred. and $\mathrm{Q} 4$ slope & 0.61 & $(0.14-2.71)$ & 2.32 & $(1.36-3.98)$ \\
\hline $\mathrm{FEV}_{1 \mathrm{~b}}<$ pred. and $\geq$ LLN and Q1 slope & 1.04 & $(0.28-3.84)$ & 2.56 & $(1.50-4.38)$ \\
\hline $\mathrm{FEV}_{1 \mathrm{~b}}<$ pred. and $\geq$ LLN and Q2 slope & 1.22 & $(0.34-4.44)$ & 2.63 & $(1.54-4.47)$ \\
\hline $\mathrm{FEV}_{1 \mathrm{~b}}<$ pred. and $\geq$ LLN and Q3 slope & 1.09 & $(0.29-4.12)$ & 3.15 & $(1.85-5.34)$ \\
\hline $\mathrm{FEV}_{1 \mathrm{~b}}<$ pred. and $\geq$ LLN and Q4 slope & 2.37 & $(0.67-8.43)$ & 5.06 & $(2.98-8.60)$ \\
\hline $\mathrm{FEV}_{1 \mathrm{~b}}<\mathrm{LLN}$ & 3.33 & $(1.01-10.93)$ & 6.92 & $(4.12-11.62)$ \\
\hline All-cause Mortality & \multicolumn{2}{|c|}{$(\mathrm{n}=1,773 ;$ Deaths $=466)$} & \multicolumn{2}{|c|}{$(\mathrm{n}=5,004 ;$ Deaths $=3,839)$} \\
\hline $\mathrm{FEV}_{1 \mathrm{~b}} \geq$ pred. and Q1 slope & 1.00 & & \multicolumn{2}{|c|}{1.00} \\
\hline $\mathrm{FEV}_{1 \mathrm{~b}} \geq$ pred. and Q2 slope & 0.83 & $(0.40-1.69)$ & 1.20 & $(0.94-1.53)$ \\
\hline $\mathrm{FEV}_{1 \mathrm{~b}} \geq$ pred. and Q3 slope & 1.59 & $(0.85-2.96)$ & 1.17 & $(0.93-1.48)$ \\
\hline $\mathrm{FEV}_{1 \mathrm{~b}} \geq$ pred. and $\mathrm{Q} 4$ slope & 1.43 & $(0.78-2.61)$ & 1.38 & $(1.11-1.73)$ \\
\hline $\mathrm{FEV}_{1 \mathrm{~b}}<$ pred. and $\geq \mathrm{LLN}$ and Q1 slope & 1.61 & $(0.90-2.87)$ & 1.48 & $(1.19-1.85)$ \\
\hline $\mathrm{FEV}_{1 \mathrm{~b}}<$ pred. and $\geq$ LLN and Q2 slope & 1.69 & $(0.95-3.02)$ & 1.72 & $(1.38-2.13)$ \\
\hline $\mathrm{FEV}_{1 \mathrm{~b}}<$ pred. and $\geq \mathrm{LLN}$ and Q3 slope & 1.58 & $(0.87-2.85)$ & 1.70 & $(1.37-2.12)$ \\
\hline $\mathrm{FEV}_{1 \mathrm{~b}}<$ pred. and $\geq \mathrm{LLN}$ and Q4 slope & 2.67 & $(1.49-4.77)$ & 2.49 & $(2.00-3.11)$ \\
\hline $\mathrm{FEV}_{1 \mathrm{~b}}<\mathrm{LLN}$ & 2.98 & $(1.71-5.19)$ & 2.85 & $(2.30-3.52)$ \\
\hline
\end{tabular}

*Adjusted for baseline age and height.

$\mathrm{FEV}_{1 \mathrm{~b}}$, baseline forced expiratory volume in one second; LLN, lower limit of normal; pred., predicted value. 


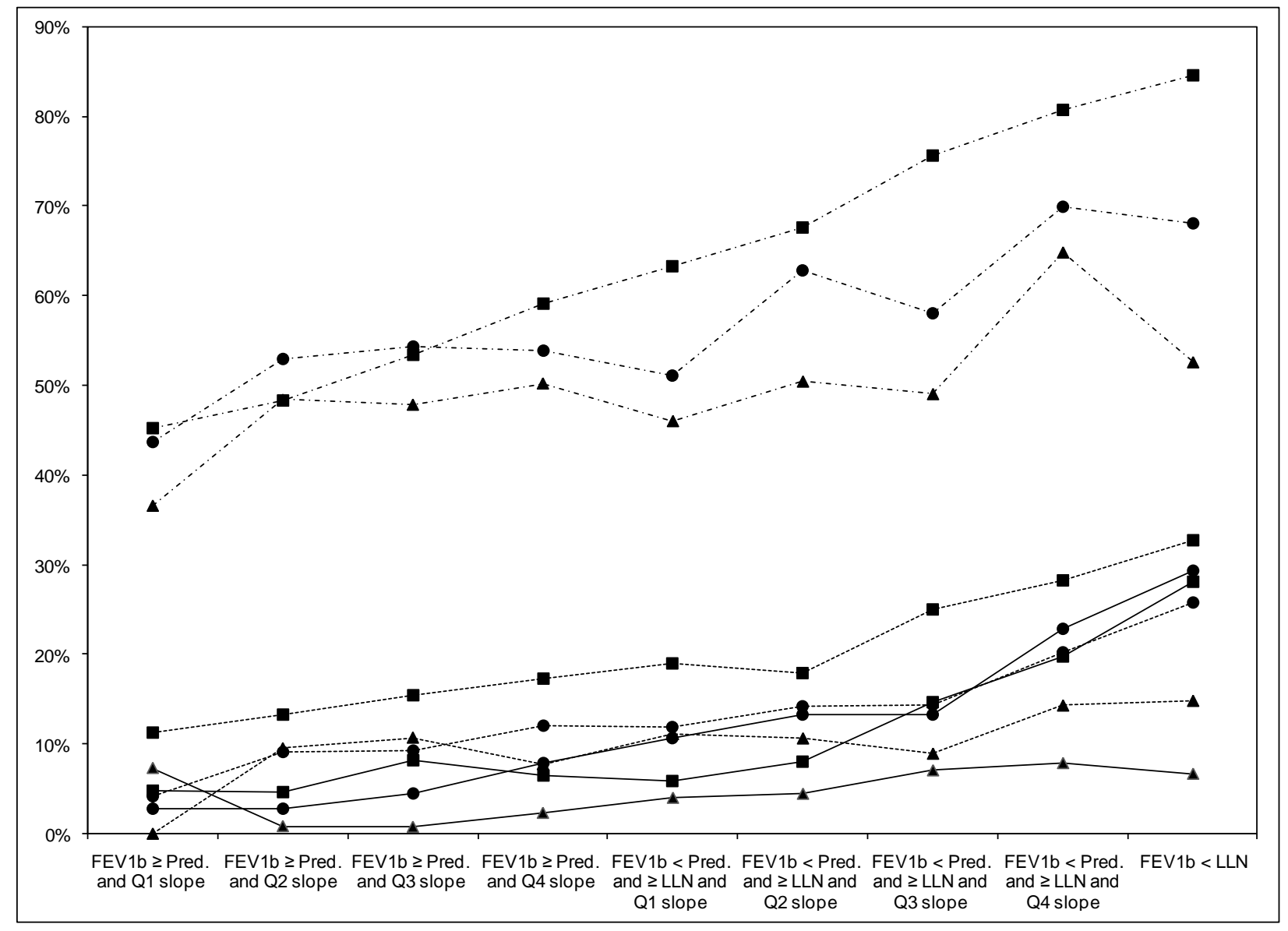

Figure C.5 Cumulative incidence by lung function category, for three subcohorts $(\boldsymbol{\bullet}=$ males; $\bullet=$ females; $\boldsymbol{\Lambda}=$ never smokers $)$ and three outcomes (COPD morbidity = solid line; COPD or CHD mortality = dashed line; all-cause mortality $=$ dash dot line). $\mathrm{FEV}_{1 \mathrm{~b}}$, baseline forced expiratory volume in one second; LLN, lower limit of normal; pred., predicted value. 


\section{References}

1. American Thoracic Society/European Respiratory Society Task Force. Standards for the diagnosis and management of patients with COPD, version 1.2.

www.thoracic.org/go/copd. Accessed February 17, 2011.

2. National Institute for Occupational Safety and Health. National Institute for Occupational Safety and Health FY 2009 Project Planning Guide. 2008.

3. Lebowitz MD. Epidemiological recognition of occupational pulmonary diseases. Clin Chest Med 1981;2(3):305-16.

4. Heederik D, Heldal KK, Artenie RC, Gorny R, Herr C, Monso E, et al. Working Group report 2: Questionnaires for work-related symptoms after organic dust exposure. Am J Ind Med 2004;46(4):414-5.

5. Hnizdo E, Glindmeyer WH. Interpretation of longitudinal pulmonary function measurements: Current state-of-the-art. Morgantown: National Institute for Occupational Safety and Health; 2008.

6. Hnizdo E, Sircar K, Yan T, Harber P, Fleming J, Glindmeyer HW. Limits of longitudinal decline for the interpretation of annual changes in $\mathrm{FEV}_{1}$ in individuals. Occup Environ Med 2007;64(10):701-7.

7. World Health Organization. COPD burden. www.who.int/respiratory/copd/burden/ en/index.html. Accessed February 2, 2011. 
8. Deaths from chronic obstructive pulmonary disease--United States, 2000-2005. MMWR Morb Mortal Wkly Rep 2008;57(45):1229-32.

9. Mannino DM, Homa DM, Akinbami LJ, Ford ES, Redd SC. Chronic obstructive pulmonary disease surveillance--United States, 1971-2000. MMWR Surveill Summ 2002;51(6):1-16.

10. Leigh JP, Romano PS, Schenker MB, Kreiss K. Costs of occupational COPD and asthma. Chest 2002;121(1):264-72.

11. Sin DD, Stafinski T, Ng YC, Bell NR, Jacobs P. The impact of chronic obstructive pulmonary disease on work loss in the United States. Am J Respir Crit Care Med 2002;165(5):704-7.

12. Balmes J, Becklake M, Blanc P, Henneberger P, Kreiss K, Mapp C, et al. American Thoracic Society Statement: Occupational contribution to the burden of airway disease. Am J Respir Crit Care Med 2003;167(5):787-97.

13. Hnizdo E, Sullivan PA, Bang KM, Wagner G. Association between chronic obstructive pulmonary disease and employment by industry and occupation in the US population: A study of data from the Third National Health and Nutrition Examination Survey. Am J Epidemiol 2002;156(8):738-46.

14. Trupin L, Earnest G, San Pedro M, Balmes JR, Eisner MD, Yelin E, et al. The occupational burden of chronic obstructive pulmonary disease. Eur Respir J $2003 ; 22(3): 462-9$. 
15. Hnizdo E, Glindmeyer HW, Petsonk EL. Workplace spirometry monitoring for respiratory disease prevention: A methods review. Int J Tuberc Lung Dis 2010;14(7):796-805.

16. Higgins ITT. Epidemiology of chronic respiratory disease: A literature review. Office of Research and Development, Environmental Protection Agency. Research Triangle Park, N.C.: Human Studies Laboratory, National Environmental Research Center; 1974.

17. Becklake MR. Chronic airflow limitation: Its relationship to work in dusty occupations. Chest 1985;88(4):608-17.

18. Becklake MR. Occupational exposures: Evidence for a causal association with chronic obstructive pulmonary disease. Am Rev Respir Dis 1989;140(3 Pt 2):S85-91.

19. Oxman AD, Muir DC, Shannon HS, Stock SR, Hnizdo E, Lange HJ. Occupational dust exposure and chronic obstructive pulmonary disease. A systematic overview of the evidence. Am Rev Respir Dis 1993;148(1):38-48.

20. Hendrick DJ. Occupation and chronic obstructive pulmonary disease (COPD). Thorax 1996;51(9):947-55.

21. Dimich-Ward H, Kennedy SM, Chan-Yeung M. Occupational exposures and chronic airflow limitation. Canadian Respiratory Journal 1996;3(2):133-140.

22. Coggon D, Newman Taylor A. Coal mining and chronic obstructive pulmonary disease: A review of the evidence. Thorax 1998;53(5):398-407. 
23. Hnizdo E, Vallyathan V. Chronic obstructive pulmonary disease due to occupational exposure to silica dust: A review of epidemiological and pathological evidence. Occup Environ Med 2003;60(4):237-43.

24. Beaty TH, Cohen BH, Newill CA, Menkes HA, Diamond EL, Chen CJ. Impaired pulmonary function as a risk factor for mortality. Am J Epidemiol 1982;116(1):102-13.

25. Beaty TH, Newill CA, Cohen BH, Tockman MS, Bryant SH, Spurgeon HA. Effects of pulmonary function on mortality. J Chronic Dis 1985;38(8):703-10.

26. Bang KM, Gergen PJ, Kramer R, Cohen B. The effect of pulmonary impairment on allcause mortality in a national cohort. Chest 1993;103(2):536-40.

27. Rodriguez BL, Masaki K, Burchfiel C, Curb JD, Fong KO, Chyou PH, et al. Pulmonary function decline and 17-year total mortality: The Honolulu Heart Program. Am J Epidemiol 1994;140(5):398-408.

28. Hole DJ, Watt GC, Davey-Smith G, Hart CL, Gillis CR, Hawthorne VM. Impaired lung function and mortality risk in men and women: Findings from the Renfrew and Paisley prospective population study. $B M J$ 1996;313(7059):711-6.

29. Ryan G, Knuiman MW, Divitini ML, James A, Musk AW, Bartholomew HC. Decline in lung function and mortality: The Busselton Health Study. J Epidemiol Community Health 1999;53(4):230-4. 
30. Schunemann HJ, Dorn J, Grant BJ, Winkelstein W, Jr., Trevisan M. Pulmonary function is a long-term predictor of mortality in the general population: 29-year follow-up of the Buffalo Health Study. Chest 2000;118(3):656-64.

31. Pelkonen M, Notkola IL, Tukiainen H, Tervahauta M, Tuomilehto J, Nissinen A. Smoking cessation, decline in pulmonary function and total mortality: A 30 year follow up study among the Finnish cohorts of the Seven Countries Study. Thorax 2001;56(9):703-7.

32. Mannino DM, Buist AS, Petty TL, Enright PL, Redd SC. Lung function and mortality in the United States: Data from the First National Health and Nutrition Examination Survey follow up study. Thorax 2003;58(5):388-93.

33. Mannino DM, Reichert MM, Davis KJ. Lung function decline and outcomes in an adult population. Am J Respir Crit Care Med 2006;173(9):985-90.

34. Mannino DM, Davis KJ. Lung function decline and outcomes in an elderly population. Thorax 2006;61(6):472-7.

35. Lange P, Nyboe J, Jensen G, Schnohr P, Appleyard M. Ventilatory function impairment and risk of cardiovascular death and of fatal or non-fatal myocardial infarction. Eur Respir J 1991;4(9):1080-7.

36. Beeckman LA, Wang ML, Petsonk EL, Wagner GR. Rapid declines in FEV ${ }_{1}$ and subsequent respiratory symptoms, illnesses, and mortality in coal miners in the United States. Am J Respir Crit Care Med 2001;163(3 Pt 1):633-9. 
37. van der Palen J, Rea TD, Manolio TA, Lumley T, Newman AB, Tracy RP, et al. Respiratory muscle strength and the risk of incident cardiovascular events. Thorax 2004;59(12):1063-7.

38. Sin DD, Wu L, Man SF. The relationship between reduced lung function and cardiovascular mortality: A population-based study and a systematic review of the literature. Chest 2005;127(6):1952-9.

39. Krzyzanowski M, Jedrychowski W, Wysocki M. Factors associated with the change in ventilatory function and the development of chronic obstructive pulmonary disease in a 13-year follow-up of the Cracow Study. Risk of chronic obstructive pulmonary disease. Am Rev Respir Dis 1986;134(5):1011-9.

40. Kauffmann F, Drouet D, Lellouch J, Brille D. Occupational exposure and 12-year spirometric changes among Paris area workers. Br J Ind Med 1982;39(3):221-32.

41. Attfield MD. Longitudinal decline in $\mathrm{FEV}_{1}$ in United States coalminers. Thorax 1985;40(2):132-7.

42. Bergdahl IA, Toren K, Eriksson K, Hedlund U, Nilsson T, Flodin R, et al. Increased mortality in COPD among construction workers exposed to inorganic dust. Eur Respir J 2004;23(3):402-6.

43. Mehta AJ, Henneberger PK, Toren K, Olin AC. Airflow limitation and changes in pulmonary function among bleachery workers. Eur Respir J 2005;26(1):133-9. 
44. Soyseth V, Johnsen HL, Benth JS, Hetland SM, Kongerud J. Production of silicon metal and alloys is associated with accelerated decline in lung function: A 5-year prospective study among 3924 employees in Norwegian smelters. J Occup Environ Med 2007;49(9):1020-6.

45. Harber P, Tashkin DP, Simmons M, Crawford L, Hnizdo E, Connett J. Effect of occupational exposures on decline of lung function in early chronic obstructive pulmonary disease. Am J Respir Crit Care Med 2007;176(10):994-1000.

46. Boggia B, Farinaro E, Grieco L, Lucariello A, Carbone U. Burden of smoking and occupational exposure on etiology of chronic obstructive pulmonary disease in workers of Southern Italy. J Occup Environ Med 2008;50(3):366-70.

47. Soyseth V, Johnsen HL, Kongerud J. Prediction of dropout from respiratory symptoms and airflow limitation in a longitudinal respiratory study. Scand $J$ Work Environ Health 2008;34(3):224-9.

48. Johnsen HL, Hetland SM, Benth JS, Kongerud J, Soyseth V. Dust exposure assessed by a job exposure matrix is associated with increased annual decline in $\mathrm{FEV}_{1}$ : A 5-year prospective study of employees in Norwegian smelters. Am J Respir Crit Care Med 2010;181(11):1234-40.

49. Centers for Disease Control and Prevention. Health People 2020 - Respiratory Diseases. http://www.healthypeople.gov/2020/topicsobjectives2020/pdfs/RespiratoryDiseases.pdf. Accessed March 7, 2011. 
50. Burge PS. Occupation and chronic obstructive pulmonary disease (COPD). Eur Respir J 1994;7(6):1032-4.

51. Townsend MC. Evaluating pulmonary function change over time in the occupational setting. J Occup Environ Med 2005;47(12):1307-16.

52. Buist AS, Vollmer WM. The use of lung function tests in identifying factors that affect lung growth and aging. Stat Med 1988;7(1-2):11-8.

53. Miller MR, Pedersen OF. New concepts for expressing forced expiratory volume in $1 \mathrm{~s}$ arising from survival analysis. Eur Respir J 2010;35(4):873-82.

54. Krzyzanowski M, Wysocki M. The relation of thirteen-year mortality to ventilatory impairment and other respiratory symptoms: The Cracow Study. Int J Epidemiol 1986;15(1):56-64.

55. Neas LM, Schwartz J. Pulmonary function levels as predictors of mortality in a national sample of US adults. Am J Epidemiol 1998;147(11):1011-8.

56. Persson C, Bengtsson C, Lapidus L, Rybo E, Thiringer G, Wedel H. Peak expiratory flow and risk of cardiovascular disease and death. A 12-year follow-up of participants in the population study of women in Gothenburg, Sweden. Am J Epidemiol 1986;124(6):942-8.

57. Tockman MS, Pearson JD, Fleg JL, Metter EJ, Kao SY, Rampal KG, et al. Rapid decline in $\mathrm{FEV}_{1}$. A new risk factor for coronary heart disease mortality. Am J Respir Crit Care Med 1995;151(2 Pt 1):390-8. 
58. Mannino DM, Homa DM, Akinbami LJ, Ford ES, Redd SC. Chronic obstructive pulmonary disease surveillance--United States, 1971-2000. MMWR Surveill Summ 2002;51(6):1-16.

59. Akazawa M, Halpern R, Riedel AA, Stanford RH, Dalal A, Blanchette CM. Economic burden prior to COPD diagnosis: A matched case-control study in the United States. Respir Med 2008;102(12):1744-52.

60. Schneider C, Bothner U, Jick SS, Meier CR. Chronic obstructive pulmonary disease and the risk of cardiovascular diseases. Eur J Epidemiol 2010;25:253-260.

61. Sin DD. Is COPD really a cardiovascular disease? Chest 2009;136(2):229-330.

62. Blanc PD, Eisner MD, Earnest G, Trupin L, Balmes JR, Yelin EH, et al. Further exploration of the links between occupational exposure and chronic obstructive pulmonary disease. J Occup Environ Med 2009;51(7):804-10.

63. Blanc PD, Iribarren C, Trupin L, Earnest G, Katz PP, Balmes J, et al. Occupational exposures and the risk of COPD: Dusty trades revisited. Thorax 2009;64(1):6-12.

64. Rodriguez E, Ferrer J, Marti S, Zock JP, Plana E, Morell F. Impact of occupational exposure on severity of COPD. Chest 2008;134(6):1237-43.

65. Weinmann S, Vollmer WM, Breen V, Heumann M, Hnizdo E, Villnave J, et al. COPD and occupational exposures: A case-control study. J Occup Environ Med 2008;50(5):561-569. 
66. Darkow T, Chastek BJ, Shah H, Phillips AL. Health care costs among individuals with chronic obstructive pulmonary disease within several large, multi-state employers. $J$ Occup Environ Med 2008;50(10):1130-8.

67. Darkow T, Kadlubek PJ, Shah H, Phillips AL, Marton JP. A retrospective analysis of disability and its related costs among employees with chronic obstructive pulmonary disease. J Occup Environ Med 2007;49(1):22-30.

68. Sircar K, Hnizdo E, Petsonk E, Attfield M. Decline in lung function and mortality: Implications for medical monitoring. Occup Environ Med 2007;64(7):461-6.

69. Schnohr P, Jensen G, Lange P, Scharling H, Appleyard M. The Copenhagen City Heart Study. Eur Heart J 2001;3(suppl_H):H1-83.

70. Lokke A, Lange P, Scharling H, Fabricius P, Vestbo J. Developing COPD: A 25 year follow up study of the general population. Thorax 2006;61(11):935-9.

71. Burrows B, Lebowitz MD, Camilli AE, Knudson RJ. Longitudinal changes in forced expiratory volume in one second in adults. Methodologic considerations and findings in healthy nonsmokers. Am Rev Respir Dis 1986;133(6):974-80.

72. Rose GA, Blackburn H. Cardiovascular survey methods. Monogr Ser World Health Organ 1968;56:1-188.

73. Miller MR, Pedersen OF, Lange P, Vestbo J. Improved survival prediction from lung function data in a large population sample. Respir Med 2009;103(3):442-8. 
74. Akaike H. A new look at the statistical model identification. IEEE Transactions on Automatic Control 1974;19(6):716-723.

75. Becklake MR, Kauffmann F. Gender differences in airway behaviour over the human life span. Thorax 1999;54(12):1119-38.

76. Prescott E, Bjerg AM, Andersen PK, Lange P, Vestbo J. Gender difference in smoking effects on lung function and risk of hospitalization for COPD: Results from a Danish longitudinal population study. Eur Respir J 1997;10(4):822-7.

77. Camp PG, O'Donnell DE, Postma DS. Chronic obstructive pulmonary disease in men and women: Myths and reality. Proc Am Thorac Soc 2009;6(6):535-8.

78. Knuiman MW, James AL, Divitini ML, Ryan G, Bartholomew HC, Musk AW. Lung function, respiratory symptoms, and mortality: Results from the Busselton Health Study. Ann Epidemiol 1999;9(5):297-306.

79. Vestbo J, Knudsen KM, Rasmussen FV. The value of mucus hypersecretion as a predictor of mortality and hospitalization. An 11-year register based follow-up study of a random population sample of 876 men. Respir Med 1989;83(3):207-11.

80. Vestbo J, Rasmussen FV. Respiratory symptoms and $\mathrm{FEV}_{1}$ as predictors of hospitalization and medication in the following 12 years due to respiratory disease. Eur Respir J 1989;2(8):710-5. 
81. Lange P, Nyboe J, Appleyard M, Jensen G, Schnohr P. Relation of ventilatory impairment and of chronic mucus hypersecretion to mortality from obstructive lung disease and from all causes. Thorax 1990;45(8):579-85.

82. Carpenter L, Beral V, Strachan D, Ebi-Kryston KL, Inskip H. Respiratory symptoms as predictors of 27 year mortality in a representative sample of British adults. $B M J$ 1989;299(6695):357-61.

83. Ebi-Kryston KL. Respiratory symptoms and pulmonary function as predictors of 10-year mortality from respiratory disease, cardiovascular disease, and all causes in the Whitehall Study. J Clin Epidemiol 1988;41(3):251-60.

84. Jensen HH, Godtfredsen NS, Lange P, Vestbo J. Potential misclassification of causes of death from COPD. Eur Respir J 2006;28(4):781-5.

85. Loeppke R, Taitel M, Haufle V, Parry T, Kessler RC, Jinnett K. Health and productivity as a business strategy: A multiemployer study. J Occup Environ Med 2009;51(4):411-28.

86. Loeppke R, Taitel M, Richling D, Parry T, Kessler RC, Hymel P, et al. Health and productivity as a business strategy. J Occup Environ Med 2007;49(7):712-21.

87. Pauwels RA, Buist AS, Calverley PM, Jenkins CR, Hurd SS. Global strategy for the diagnosis, management, and prevention of chronic obstructive pulmonary disease. NHLBI/WHO Global Initiative for Chronic Obstructive Lung Disease (GOLD) Workshop summary. Am J Respir Crit Care Med 2001;163(5):1256-76. 
88. Doherty DE. A review of the role of $\mathrm{FEV}_{1}$ in the COPD paradigm. COPD 2008;5(5):310-8.

89. Ferguson GT, Enright PL, Buist AS, Higgins MW. Office spirometry for lung health assessment in adults: A consensus statement from the National Lung Health Education Program. Chest 2000;117(4):1146-61.

90. Hankinson JL, Wagner GR. Medical screening using periodic spirometry for detection of chronic lung disease. Occup Med 1993;8(2):353-61.

91. Global Initiative for Chronic Obstructive Lung Disease. Global strategy for the diagnosis, management, and prevention of chronic obstructive pulmonary disease. http://www.goldcopd.com/. Accessed January 7, 2011.

92. Chapman KR, Mannino DM, Soriano JB, Vermeire PA, Buist AS, Thun MJ, et al. Epidemiology and costs of chronic obstructive pulmonary disease. Eur Respir J 2006;27(1):188-207.

93. Murray CJ, Lopez AD. Alternative projections of mortality and disability by cause 19902020: Global Burden of Disease Study. Lancet 1997;349(9064):1498-504.

94. Yelin E, Katz P, Balmes J, Trupin L, Earnest G, Eisner M, et al. Work life of persons with asthma, rhinitis, and COPD: A study using a national, population-based sample. $J$ Occup Med Toxicol 2006;1(2):1-9.

95. Anthonisen NR, Connett JE, Murray RP. Smoking and lung function of Lung Health Study participants after 11 years. Am J Respir Crit Care Med 2002;166(5):675-9. 
96. Kohansal R, Martinez-Camblor P, Agusti A, Buist AS, Mannino DM, Soriano JB. The natural history of chronic airflow obstruction revisited: An analysis of the Framingham offspring cohort. Am J Respir Crit Care Med 2009;180(1):3-10.

97. Kalhan R, Arynchyn A, Colangelo LA, Dransfield MT, Gerald LB, Smith LJ. Lung function in young adults predicts airflow obstruction 20 years later. Am J Med 2010;123(5):468 e1-7.

98. Baughman P, Marott JL, Lange P, Andrew M, Hnizdo E. Health outcomes associated with lung function decline and respiratory symptoms and disease in a community cohort. COPD 2011;8(2):103-13.

99. Lange P, Mogelvang R, Marott JL, Vestbo J, Jensen JS. Cardiovascular morbidity in COPD: A study of the general population. COPD 2010;7(1):5-10.

100. Eisen EA, Agalliu I, Thurston SW, Coull BA, Checkoway H. Smoothing in occupational cohort studies: An illustration based on penalised splines. Occup Environ Med 2004;61(10):854-60.

101. Hnizdo E, Glindmeyer HW, Petsonk EL, Enright P, Buist AS. Case definitions for chronic obstructive pulmonary disease. COPD 2006;3(2):95-100.

102. Lash TL, Johansen MB, Christensen S, Baron JA, Rothman KJ, Hansen JG, et al. Hospitalization rates and survival associated with COPD: A nationwide Danish cohort study. Lung 2011;189(1):27-35. 
103. Bilde L, Rud Svenning A, Dollerup J, Baekke Borgeskov H, Lange P. The cost of treating patients with COPD in Denmark--a population study of COPD patients compared with non-COPD controls. Respir Med 2007;101(3):539-46.

104. Hogg JC, Pierce RA. Remodelling of peripheral lung tissue in COPD. Eur Respir J 2008;31(5):913-4.

105. Black PN, Ching PS, Beaumont B, Ranasinghe S, Taylor G, Merrilees MJ. Changes in elastic fibres in the small airways and alveoli in COPD. Eur Respir J 2008;31(5):9981004.

106. Allen TC. Pathology of small airways disease. Arch Pathol Lab Med 2010;134(5):70218.

107. Cornwell WD, Kim V, Song C, Rogers TJ. Pathogenesis of inflammation and repair in advanced COPD. Semin Respir Crit Care Med 2010;31(3):257-66.

108. Sin DD, Man SF. Why are patients with chronic obstructive pulmonary disease at increased risk of cardiovascular diseases? The potential role of systemic inflammation in chronic obstructive pulmonary disease. Circulation 2003;107(11):1514-9.

109. ACOEM Occupational and Environmental Lung Disorder Committee. ACOEM position statement: Spirometry in the occupational health setting-2010 update. In press 2010.

110. Lange P. Spirometric findings as predictors of survival. Thorax 2011;66(1):1-2.

111. Quanjer PH, Tammeling GJ, Cotes JE, Pedersen OF, Peslin R, Yernault JC. Lung volumes and forced ventilatory flows. Report Working Party Standardization of Lung 
Function Tests, European Community for Steel and Coal. Official statement of the European Respiratory Society. Eur Respir J Suppl 1993;16:5-40.

112. Levin ML. The occurrence of lung cancer in man. Acta Unio Int Contra Cancrum $1953 ; 9(3): 531-41$.

113. Hnizdo E, Yan T, Enright P, Beeckman-Wagner L, Hankinson J, Fleming J, et al. Spirometry Longitudinal Data Analysis software (SPIROLA) for analysis of spirometry data in workplace prevention or COPD treatment. The Open Medical Informatics Journal 2010;4(1):94-102.

114. American Thoracic Society. Standardization of spirometry, 1994 update. American Thoracic Society. Am J Respir Crit Care Med 1995;152(3):1107-36.

115. Camp PG, Goring SM. Gender and the diagnosis, management, and surveillance of chronic obstructive pulmonary disease. Proc Am Thorac Soc 2007;4(8):686-91.

116. Burney PG, Hooper R. Forced vital capacity, airway obstruction and survival in a general population sample from the USA. Thorax 2011;66(1):49-54. 